\title{
Analysing Linear Spatial Relationships: The Measures of Connectivity, Integration, and Choice
}

\section{Contents}

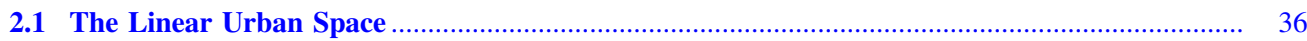

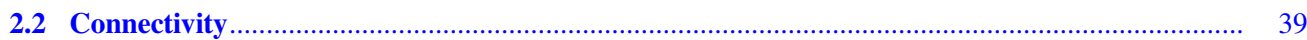

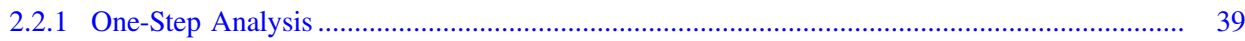

2.2.2 Two-Step Analysis ................................................................................................... 41

2.2.3 Three-Step and N-Step or Point-Depth Analyses.............................................................. 44

2.3 Global Axial Integration Analysis ...................................................................................... 46

2.3.1 The Mathematical Formulas for Calculating Global Integration ............................................ 48

2.3.2 Global Spatial Integration and Segregation ........................................................... 50

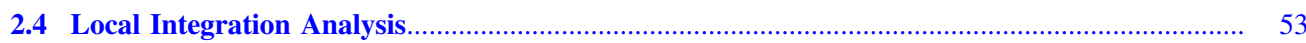

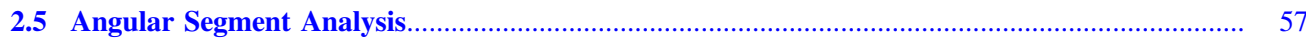

2.5.1 Radii Used in Segment Analysis ................................................................................... 62

2.5.2 Angular Choice: Main Routes Through Cities and Regions ............................................... 66

2.5.3 Angular (Segment) Integration: The Location of Urban Centres............................................ 70

2.5.4 A More Elaborate Explanation for How to Calculate Angular (Segment) Integration and Choice.

2.5.5 Normalising Angular Integration and Angular Choice Values: NAIN and $\mathrm{NACH}$.

2.6 Four-Pointed Star Model.

2.7 The Use of Scatterplots

2.8 Conclusions: Common Errors and Mistakes When Analysing Linear Urban Space

2.9 Exercises

References

Further Readings

\section{Abstract}

In this chapter, we first explain the concept of an axial line and how the axial map is applied in space syntax. We then discuss the static measure of 'connectivity' with its 'one-step' to 'n-step' logic, including its meaning for axial integration analysis. We further present the segment integration analysis. Using the street segment as the basis for analysis allows one to apply three types of distances and three types of radii in space syntax. We then present the most-often used space syntax measures in more depth, namely angular choice and angular integration with metric radius, and introduce the 
mathematical formulae on how to normalise both measures. Real-life applications illustrate and underpin the usefulness of these measures and their meaning for urban analysis, such as why and how they allow us to identify urban societal processes and their added value at both a citywide scale and a neighbourhood scale. Finally, we critically reflect on the measures, including their potentials and misfits. Exercises are provided at the end of the chapter.

\section{Keywords}

Street network analysis $\bullet$ Graph theory $\bullet$ Connectivity $\bullet$ Integration $\bullet$ Choice $・$ To-movement $\bullet$ Through-movement

\section{Key concepts}

Graph theory $\bullet$ Axial map $・$ Segment map $\bullet$ Topological step $\bullet$ Topological distance $\bullet$ Geometric distance $\cdot$ Angular distance • Integration • Angular choice • To-movement $\bullet$ Through-movement

\section{Learning objectives}

After studying this chapter, you will be

- able to draw an axial map;

- able to carry out an n-step analysis and interpret its results;

- able to process from an axial map the measures of axial integration, segment integration, and choice with different radii for the same and different measures;

- able to normalise space syntax numerical values;

- able to undertake and interpret correlations between the same measures with different radii and between different measures;

- able to generate a four-pointed star model based on Hillier's fifty-cities approach; and

- able to critically reflect upon all obtained analytical results.

\subsection{The Linear Urban Space}

Independent of cultures and architectural styles, all cities have in common is that they consist of a set of private and public spaces. Public spaces are accessible for all and open up for movement from everywhere to everywhere else in the built environment. Private spaces have restricted access and are mostly inside buildings or can be private gardens. Private spaces are connected to public spaces in different degrees of depth, and the way all public and private spaces are interconnected conditions the possibilities for people's socio-economic activities.

The urban street network is constituted by public spaces. Puckett (2009) states that the street structure affects how human beings navigate and orientate themselves in urban space. When, for example, escaping from a threat, a network-structured street grid offers more escape possibilities than a tree-structured grid (Marshall 2005). In a network-structured street grid, the choices are many, whereas they can be quite limited in a tree-structured street grid, in particular for dead-end streets (see Chap. 1). In panic situations, rational thinking is reduced, and thus visual orientation has a high priority when people have to escape (Puckett 2009, p. 22). For example, in the 2004 tsunami in the city of Banda Aceh, Indonesia, mortality rates were ten times higher in neighbourhoods with a tree-structured street grid compared to neighbourhoods with a network-structured street grid (Fakhurrazi and van Nes 2012). Therefore, the axial sightline matters for human navigation in panic situations as well as in daily life. Seemingly, people follow their nose in the way they orientate through built environments (Dalton 2001). Therefore, it is worth focusing on the role of the axial sightline as a basic element for the analysis of the street network.

Urban public space is mostly 'linear'. Except for squares, the streets, roads, paths, avenues, pavements, or boulevards are linear items. Therefore, each spatial element of the street network can be represented as an axial sightline indicating movement paths. The axial line is a sightline with an underlying movement logic. To produce an axial map, all roads, streets, paths, subways, bridges, and tunnels are modelled in the same way using an axial line. The road width per se is not taken into account and is only indirectly measured in that the length of the axial line picks up the width of the street. For two streets with the same length, the wider street will have the longer axial line compared to the narrower street. As a basic tool, space 
syntax applies the axial map as the minimal set of longest straight sightlines indicating movement interconnecting all open spaces (Yamu and Voigt 2011; Yamu et al. 2021). In other words, the axial map represents urban space that is possible to visually overlook and physically access (Marcus 2007). Marshall claims that the axial line reflects the geometry of bounded space (Marshall 2005). An axial map is the geometric model of an urban street network that can translate into a topological graph (see Chap. 1).

Because the space syntax analysis utilises a graph-theory approach, the axial analysis examines the movement routes' degrees of (inter)connectivity. For a strategic city model, whether a street incorporates a tram or bus line or is pedestrianised or only accessible for public transport is not at stake here. All streets and roads are treated in the same manner and fashion for generating an axial map. Metro lines, railways, and light rail tracks are not taken into account in the axial map when they are separated from a street or road network. Human movement for metro and train services are regulated by introduced frequencies and predefined routes. Nevertheless, the location of public transport stops and stations in the street network is of interest. How these are accessible to their surroundings matters for making public transport accessible.

Figure 2.1 illustrates an example of how urban space can be abstracted and represented with a set of axial lines (b) and segments (c) for the Ferdinand Bolstraat area in Amsterdam (a). For each public space, an axial line is drawn. Thus, an urban street network is represented by the longest and fewest set of axial lines interconnecting with one another. With the axial map, we abstract and simplify reality. For the segment map, the axial map is broken up into segments at each street intersection.

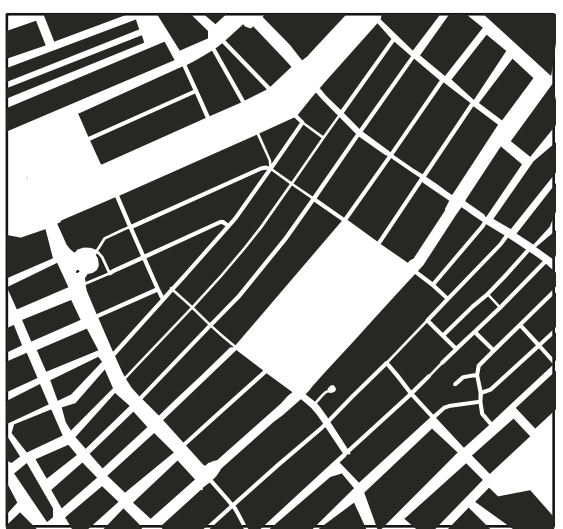

(a) Figure-ground diagram

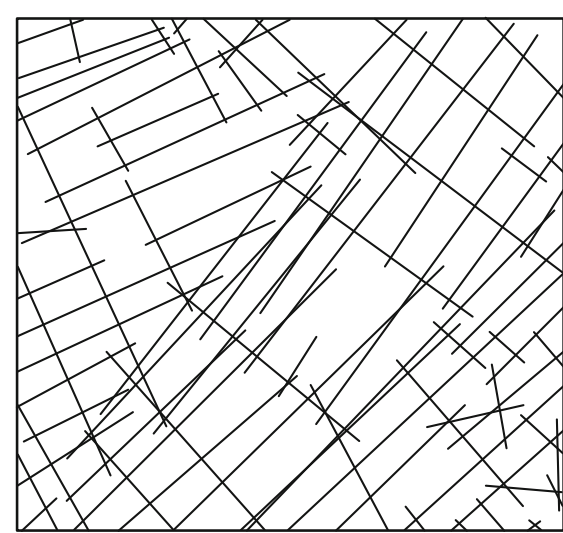

(b) Axial representation

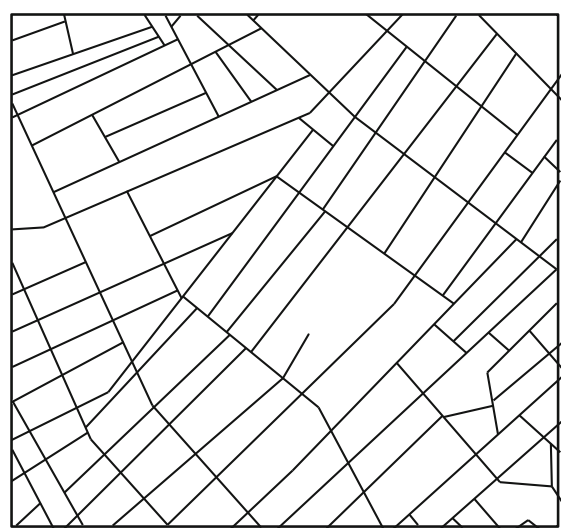

(c) Segment representation

Fig. 2.1 A figure ground diagram (a), an axial (b), and a segment (c) representation of the Ferdinand Bolstraat area in Amsterdam

The basis for drawing an axial map can be any kinds of urban maps, as long as the public open spaces are visible. At present in the era of digital services, Google Maps, Bing Maps, and similar products can be used as base maps. Historic maps have to be digitalised to create, for example, discrete space syntax models through time. Once a digital version of an urban map is available, the axial map can be drawn using a variety of software, including Geographic Information System (GIS), Adobe Illustrator, AutoCAD, or Vectorworks. In general, any software can be used that allows one to draw and export .dxf files or interchangeable formats such as .mif files. The advantage of GIS is that the map is georeferenced, which is useful for later correlations with socio-economic data.

An axial map should always be drawn on a separate layer. When drawing an axial map, some simple rules have to be followed. All streets, roads, paths, etc. are equally represented as a set of the longest and fewest number of axial lines. At every direction change, make sure that the individual axial lines (in the latter, we will also call them 'axes') overlap well. Even though the axial stubs look 'ugly' at the junctions and in the bends of curved streets, it is better than to have an axial map with streets that are disconnected where they should not be.

Figure 2.2 shows some typical beginner's errors. The first error is to draw too many axial lines for a street. When the street is straight or its width varies, it is the longest through vision that is represented as one continuous line. Yet another new beginner error is to draw too many axes in curved streets. The second error is that streets are poorly linked because the connecting axes lack the axial stubs. Streets with a large-radius bend need to have long axial stubs to ensure that they are connected when importing the axial map into the space syntax software. The third error is to represent squares as 'objects'. The axes from streets connected to squares need to be drawn over the squares until they reach a physical obstacle like a building or a fountain. 
Roundabouts can be represented in several ways. The only exception is when there is an object blocking the through vision, like a column or a tower (see below in Fig. 2.2). The same accounts for complex highway junctions. In most cases, a single axis can be used for highways with several lanes. The advantage is less time used for 'unlinking' (see explanation below) the highways from the local streets. A spaghetti junction can be presented either as two single axes crossing each other or by representing each fly-over with axes. The advantage of representing a spaghetti junction with two axes crossing each other is that there is no need to unlink these lines. All roads are in some way connected to each other.

Fig. 2.2 Some typical beginner's errors and general advice for drawing an axial map

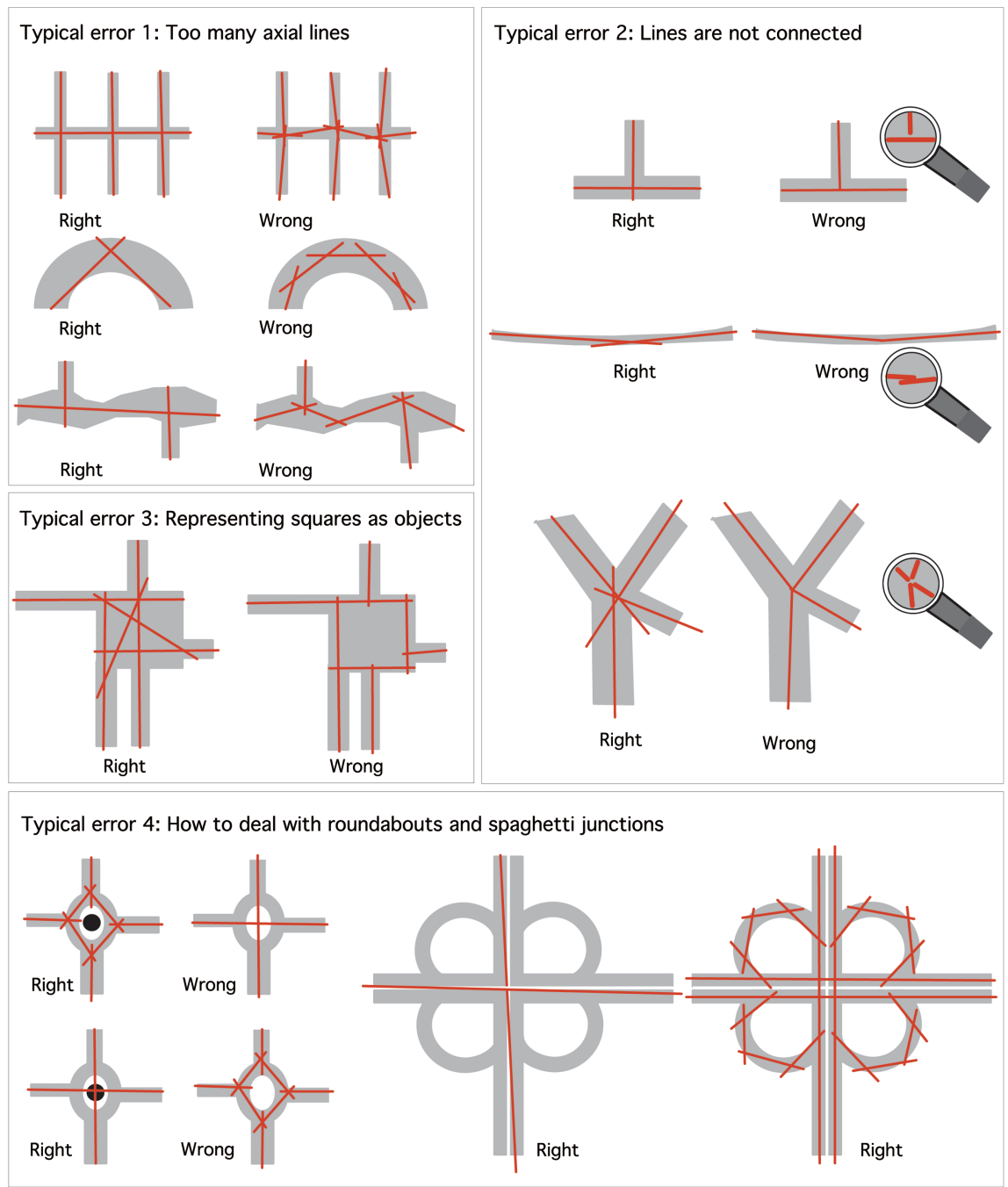

However, axial maps can be drawn in different resolutions with more or less detail. This depends on the scale level you are interested in, the focus of movement - for example, car versus pedestrian movement — and the (research) question under scrutiny. The quantum of the information influences the drawing and analysis of the axial map. An axial map can be of low, medium, or high resolution. A low-resolution model includes only the major relationships between aspects of the built environment. A medium-resolution model extends this by adding more pragmatic relations of the built and unbuilt environment. This can be, for example, a column or water feature in a public space that is taken into account. The major difference in the high-resolution axial map to all others is that the sidewalk and street crossings will be the focal point for drawing the axial map. This kind of axial map is used to generate local pedestrian models.

Another important feature in space syntax is to 'unlink' crossing axial lines that should not be connected. In reality, urban space often consists of complex overlapping axial lines that might not connect to each other, even though they look like they would in the plan. Overpasses, underpasses, tunnels, and stairways are good examples. If an axial map links overlapping lines that do not in reality connect, the analysis would be skewed and inaccurate. To deal with these conditions, it is possible to 'unlink' overlapping lines that do not share links in reality in the space syntax software (Space Syntax Ltd 2004). 
In the following, we introduce some simple static spatial analysis techniques to illustrate the various degrees of connectivity a particular street has to its vicinity. In mathematics, connectivity is one of the basic concepts of graph theory. In space syntax, connectivity is applied to explain the number of connections each street has to its direct neighbouring streets. These simple static spatial analyses can be done manually as well as by using software.

\subsection{Connectivity}

Connectivity is a static local measurement, and it accounts for all direct connections each street has to other streets in its immediate vicinity. A street with many connections to its side streets has a high connectivity value, whereas a street with a few connections has a low connectivity value. These values can be visualised using a colour range. For example, the degree of connectivity for each street in the town of Mannheim in Germany is visualised on a map in Fig. 2.3. The axial lines marked in red and yellow are the streets with the highest numbers of connections to adjacent streets. All the streets with dark blue colours have only one or two connections in their vicinity. Streets in the city centre have high connectivity values. One of Mannheim's main shopping streets, Kurpfalzstraße, is the street with one of the highest connectivity values. This northsouth-orientated street runs through the middle of Mannheim's city centre. Likewise, Kurpfalzstraße's side streets also have high values. The street with the highest connectivity (in the north of the city) is a local residential street. Its side streets have very low connectivity.

Fig. 2.3 Mannheim's connectivity graph

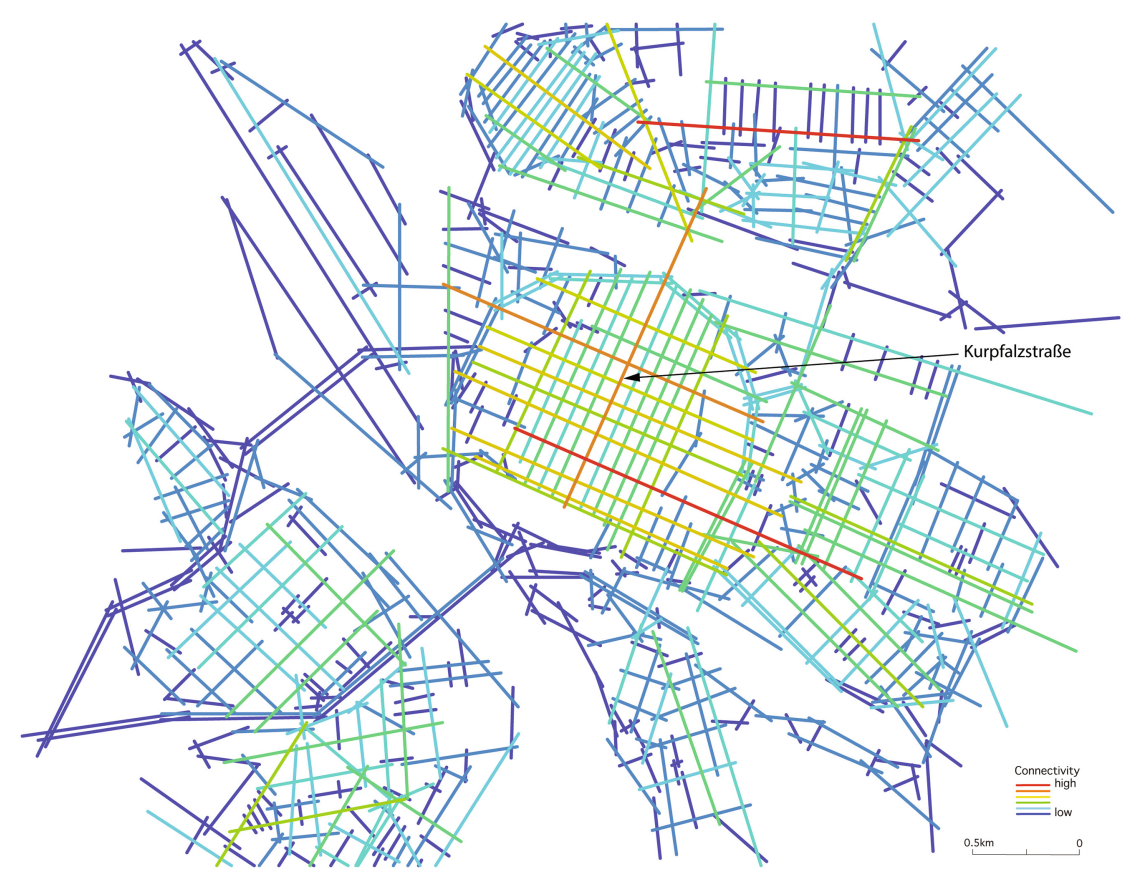

Connectivity analysis is thus very simple. It is about counting the number of connections each street has to its adjacent streets. In the following, we will demonstrate some more advanced connectivity analyses.

\subsubsection{One-Step Analysis}

This type of analysis is also a connectivity analysis, and it demonstrates how directly a street or road is connected to its vicinity. Figure 2.4 depicts a one-step analysis from a random residential street (a) and shopping street (b) as the origin in the Ferdinand Bolstraat area in Amsterdam. They are indicated in red. The residential street has two direct connections, whereas the shopping street has 25 direct connections to other streets. These connected streets are coloured in green. The shopping street consists of two axial lines with the reasoning that the street is curved. The shopping street can also be represented as one curved street segment. 
Fig. 2.4 Examples of one-step analyses in the Ferdinand Bolstraat area in Amsterdam
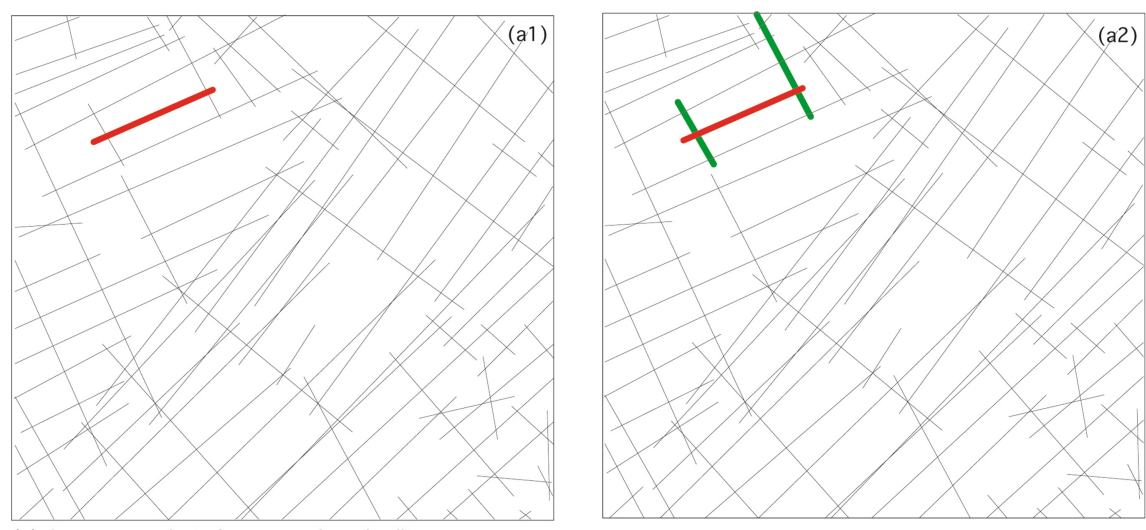

(a) One step analysis from a random dwelling street

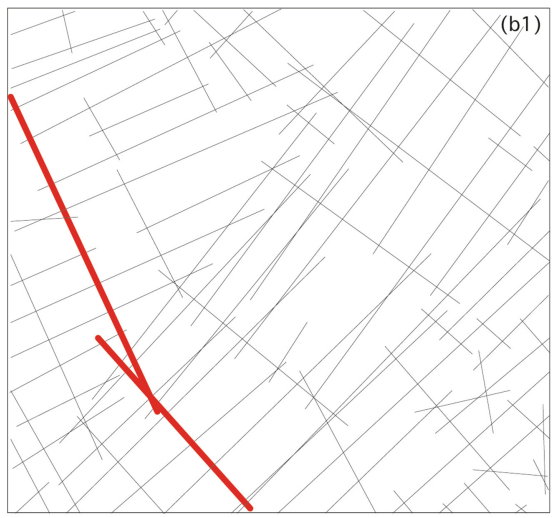

(b) One step analysis from Ferdinand Bolstraat

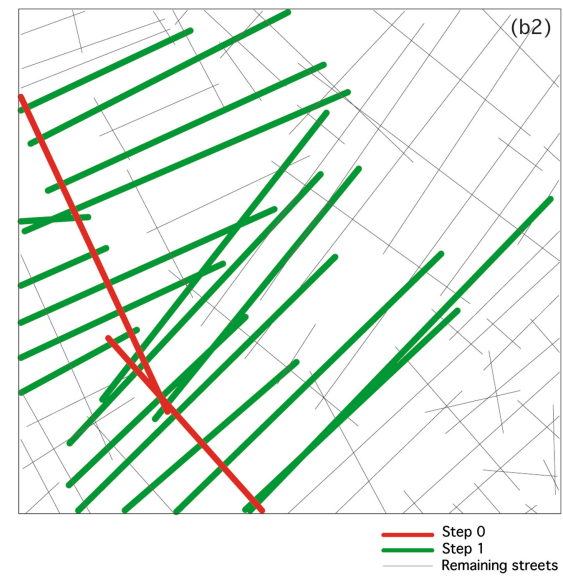

The higher the number of axial lines directly connected to the street under scrutiny, the more this street is connected to its surroundings. The one-step analysis is useful for understanding the degree of connectivity of chosen streets to their vicinity.

Main routes are defined as routes people choose to travel through and between urban areas (van Nes 2009). For a city, main routes can be identified by highlighting all long streets whose endings abut long streets at an angle close to $180^{\circ}$ Herein, Figuradeiro (2005, p. 165) defines a continuous line with the following rule of thumb: Streets that are connected to each other with an angle of $145-215^{\circ}$ or streets that deviate less than $35^{\circ}$ from a straight line are considered as 'continuous' lines. This insight builds upon the line of thought of Klaus Humpert that pedestrians have an 'ideal movement line' to reach their end point. According to Humpert (2007), if the ideal movement line deviates from the end point by more than $20^{\circ}-30^{\circ}$ off the existing movement line, the pedestrian re-orientates his/her direction. This is how path branching develops. Most old boulevards, main streets, and main distributors tend to have long visual sightlines indicating movement paths. Figure 2.5 illustrates a one-step analysis for main routes in the Pijp and Bijlmermeer areas in Amsterdam. 
Fig. 2.5 One-step analyses of the main routes in the Pijp (a) and Bijlmermeer (b) areas in Amsterdam. The Pijp neighbourhood is a nineteenth-century built-up polder area, and Bijlmermeer is a modernist neighbourhood, planned and built in the 1960 s

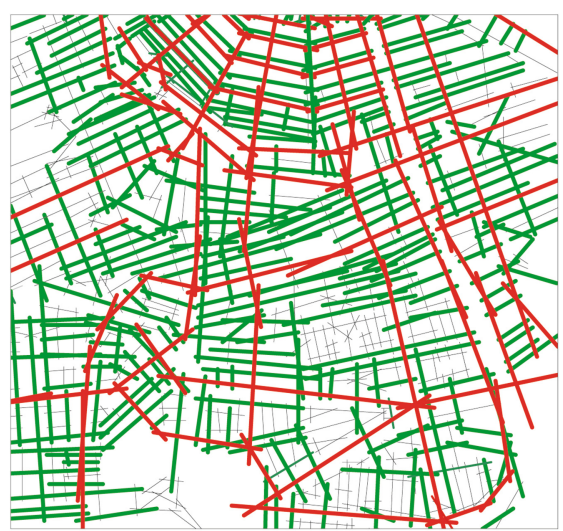

(a) Section of the Pijp area in Amsterdam

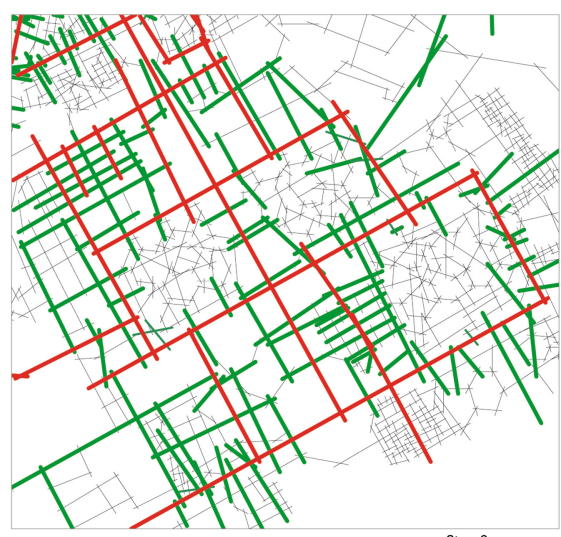

(b) Section of the Bijlmermeer area $=$ Step 0 in Amsterdam

For both the Pijp and Bijlmermeer areas, we marked all of the longest sightlines indicating paths in red (step zero). All connecting streets to the indicated streets in red are marked in green. These represent the one-step analysis for the main route network. All remaining streets are indicated in grey. From the one-step analysis, we see that the number of green axial lines connected to the main route network is high in the Pijp area. Further, Pijp's main route network itself has a high density. Therefore, this neighbourhood is a topologically 'shallow' system because it is well interconnected. All destinations from all origins can be easily reached. Thus, the number of remaining streets indicated in grey is low. For this area, by changing the direction only once or twice, a main route can be reached.

In contrast, for the Bijlmermeer area, the main route network is more fragmented and has a lower density than the Pijp area. Undertaking the one-step analysis, the axial lines in green also depict a fragmented pattern. The number of remaining axial lines is higher than in the Pijp neighbourhood. Compared to the Pijp area, the Bijlmermeer area is a topologically 'deep' system. It requires a high number of direction changes to reach the main route network.

This analysis demonstrates the interconnective relationship between main routes and local street networks. Historic and pre-war urban areas have a distinct street hierarchy established, and the main route system can be easily identified on a normal street map. For a traditional European city, the main routes often form a 'deformed wheel'. The main route network functions as the urban backbone, and major streets lead through urban local centres, whereas for a number of modernist areas, the main route network bypasses the local centres and they become suburban, poorly accessible pockets.

\subsubsection{Two-Step Analysis}

This type of analysis allows one to depict the local catchment area for two direction changes in the network from a chosen set of axial lines. The visualised 'two-step grid' shows the degree of accessibility to the surrounding neighbourhood. This analysis can be manually carried out by indicating in a colour the chosen streets under scrutiny (step zero). In the next step applying the one-step analysis logic, all connecting streets are marked in a different colour (step one) - here coloured in green. Now from these streets again, all connecting streets are marked in another colour (step two).

In our examples for a residential and a shopping street in Fig. 2.6, the streets located two topological steps or two direction changes away from the origin streets are marked in blue. The sum of all axial lines of step one and step two comprises the catchment area for the chosen streets indicated in red. For the shopping street (Fig. 2.6), the local catchment area of the two-step analysis covers most of the street network in comparison with the one-step analysis (Fig. 2.4). 
Fig. 2.6 Examples of how to conduct a two-step analysis for a residential street (a) and a shopping street (b) in Ferdinand Bolstraat in Amsterdam
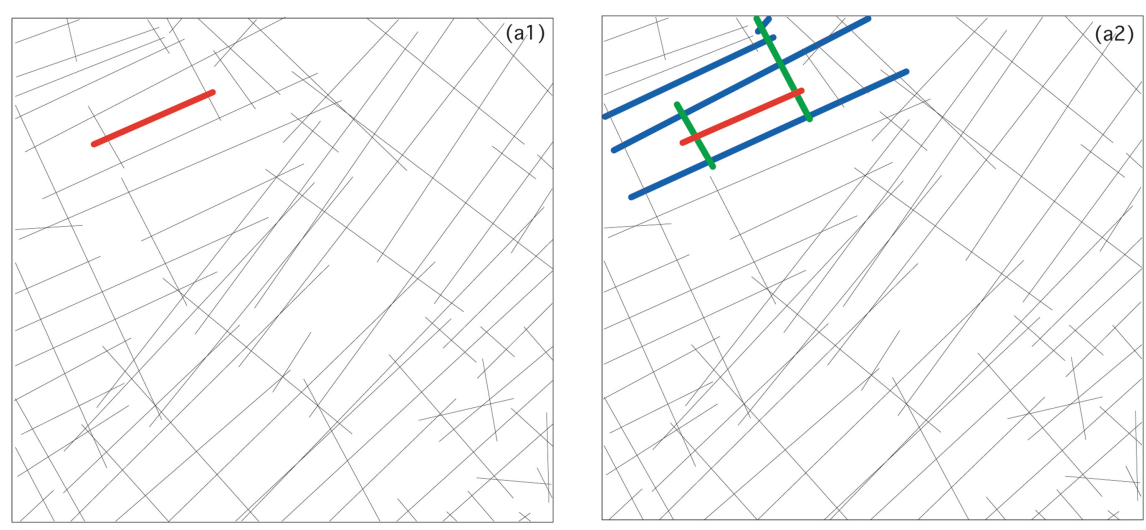

(a) Two-step analysis from a random dwelling street

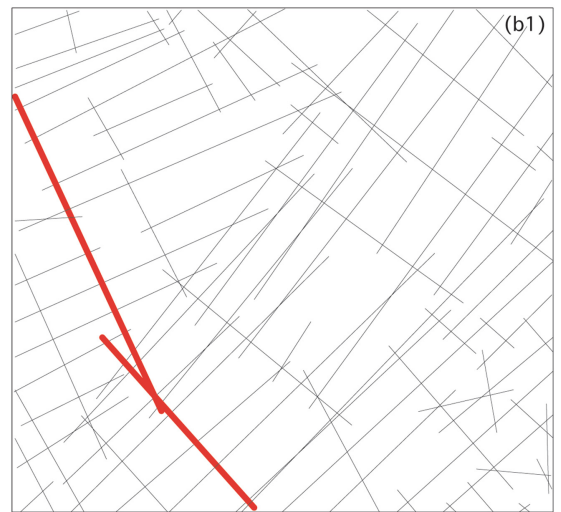

(b) Two-step analysis from Ferdinand Bolstraat

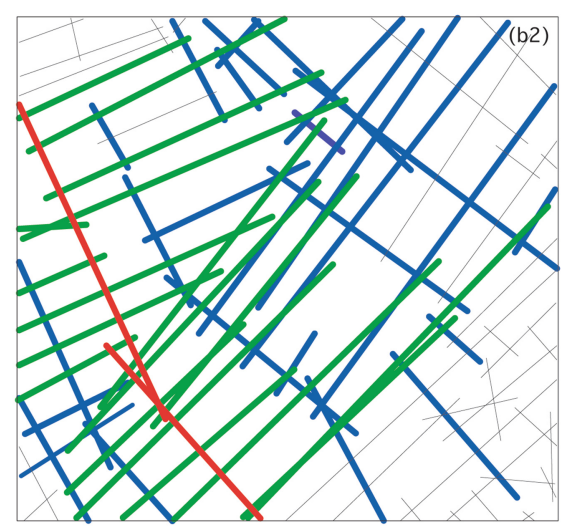

Step 0
Step 1
Step 2

Streets covering most of an area's street network with a topological 'two-step grid' (Hillier 1999, p. 119) within short metric distances are often an area's vital local shopping streets. For Hillier, for the disposition of shops, the density of the streets inside the two-step grid within short metric distances is what matters, not the length of the axial lines. This simple static spatial analysis can be applied for testing the degree of connectivity and therefore the accessibility a local shopping street has in its neighbourhood.

However, two types of urban patterns for shopping areas can be distinguished in Fig. 2.7: (left) a linear pattern and (right) a cluster pattern. For a linear pattern, the shops are aligned alongside a street with high connectivity to its vicinity with a short metric distance. Shopping in such a shopping area is mostly convenient and efficient on foot, bicycle, or public transport. In city centres, this type of street is often pedestrianised as an integral part of a pedestrian zone. This linear urban pattern type often appears in traditional, naturally growing cities and neighbourhoods.

For the cluster pattern, the shops tend to be located in modernistic areas where the buildings are not grouped as urban blocks. The shops are clustered in a designated area with short metric distances between the shops. This area is highly accessible by car. Independent of their size, these shopping areas tend to provide an open square with some benches. The larger ones have copied the narrow labyrinthine street pattern of an old historic town centre. Some shopping areas have only indoor facilities (and these often translate into shopping malls and shopping centres). These indoor spaces are only accessible during the shops' openings hours. This shopping experience for the clustered pattern of shops is mostly car-based because the shopping tour begins and ends at the car's parking spot.

Our example in Fig. 2.7 depicts Ferdinand Bolstraat and the Oostpoort shopping centre in Amsterdam to illustrate both types of urban patterns for shopping as active land use. Ferdinand Bolstraat is a local shopping street for the large and densely populated Pijp neighbourhood inside the ring road of Amsterdam. Moreover, this shopping street is also the main route running through the Pijp neighbourhood towards the city centre. Large numbers of cars, trams, bicycles, and pedestrians frequent this linear shopping street. 
Compared to Ferdinand Bolstraat, Oostpoort has a low density of axial lines in its vicinity. This indicates larger block sizes and therefore a pedestrian-unfriendly environment in the vicinity of the shopping centre. Inside the shopping centre, some artificial short blocks are made. The spaces between these blocks are only accessible to the public during the shopping centre's opening hours. The Oostpoort shopping centre is located close to regional highways (van Nes 2005).

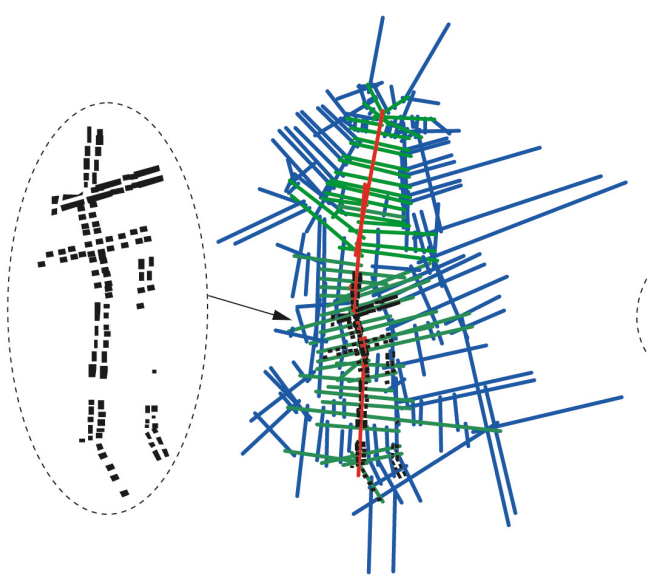

(a) Ferdinand Bolstraat

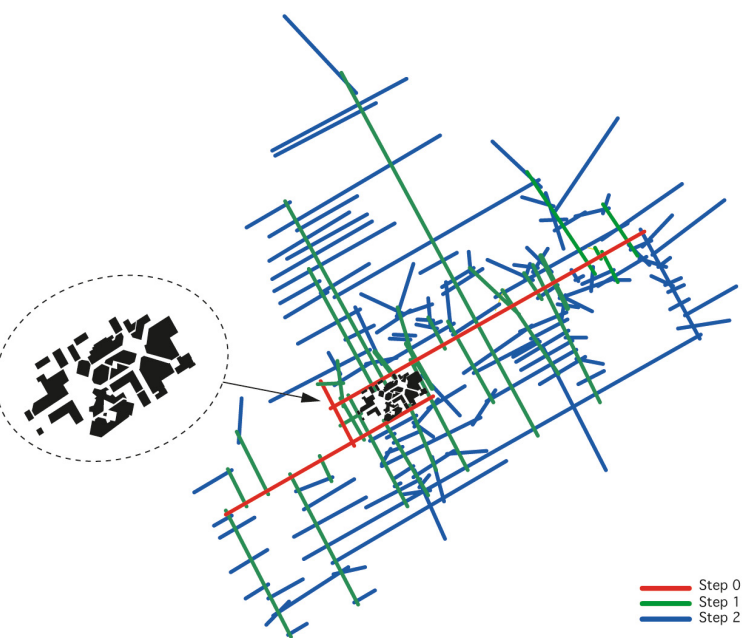

(b) Amsterdamse Oostport

Fig. 2.7 Spatial features of an urban linear (a) and cluster (b) pattern for shopping

The higher the street network density for the two-step grid for a shopping street, the higher the number and variety of shops. The types of shops might change in line with how society changes, whereas some types of shops might disappear due to online shopping, new types of shops or commercial activities will emerge on their spots as long as there is no economic regression. Aside from the influence of online shopping on current land uses, the rule of thumb is that diverse active land use emerges along streets with many connections to their vicinity. In contrast, clustered shopping areas, shopping outlets, and shopping malls are connected to the regional scale, but they serve both the local and regional scales. A clustered shopping area is also connected to surrounding residential areas to reach local and regional customers. A shopping mall's location is often adjacent or in close proximity to the main routes in a city. On a citywide scale, emerged historic main streets and shopping streets have a high number of connections to other streets. Thus, their location is central to the urban system. The more the local shopping streets are, the more they depend on a strategic spatial position in the neighbourhood. A two-step analysis shows in what way shopping streets and shopping areas are centrally located. Figure 2.8 shows the public realm of the Oostpoort shopping centre (left) and Ferdinand Bolstraat (right) in Amsterdam.

Fig. 2.8 Images of the public realm of Oostpoort shopping centre (left) and Ferdinand Bolstraat (right)
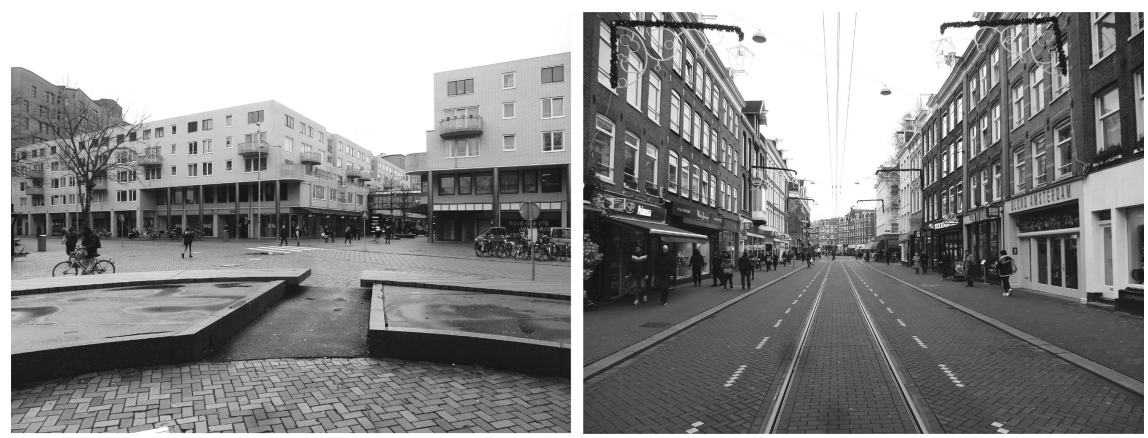


\subsubsection{Three-Step and N-Step or Point-Depth Analyses}

To recapitulate, the one-step and two-step analyses demonstrate how a particular street or road is connected to other streets or roads in its direct vicinity. For the analysis, the number of syntactic steps from a particular axial line or street segment can be increased. Figure 2.9 shows how to conduct a three-step analysis using our previous example of a residential and shopping street. The three-step analysis for the shopping street shows that the 'three-step grid' covers almost every street in the neighbourhood, whereas for the residential street the third syntactical step is the shopping street itself.

Fig. 2.9 Examples of how to conduct a three-step analysis for a residential street (a) and a shopping street (b) in Ferdinand Bolstraat in Amsterdam

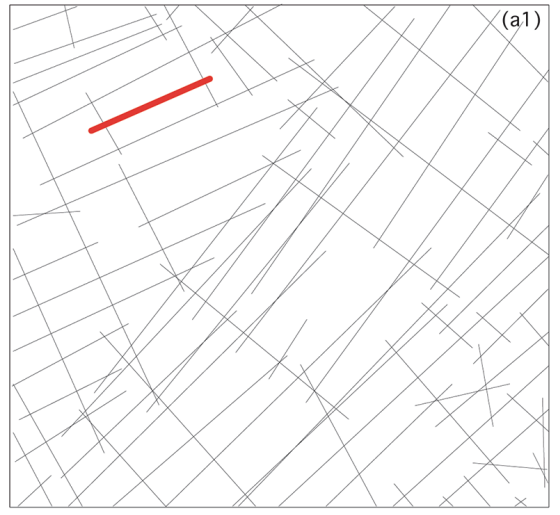

(a) Three-step analysis from a random dwelling street

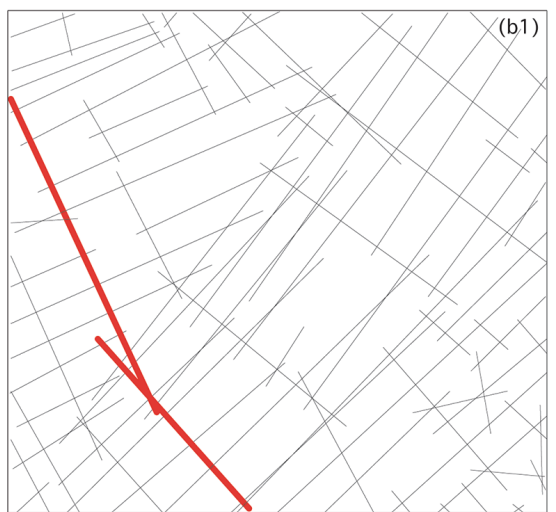

(b) Three-step analysis from Ferdinand Bolstraat
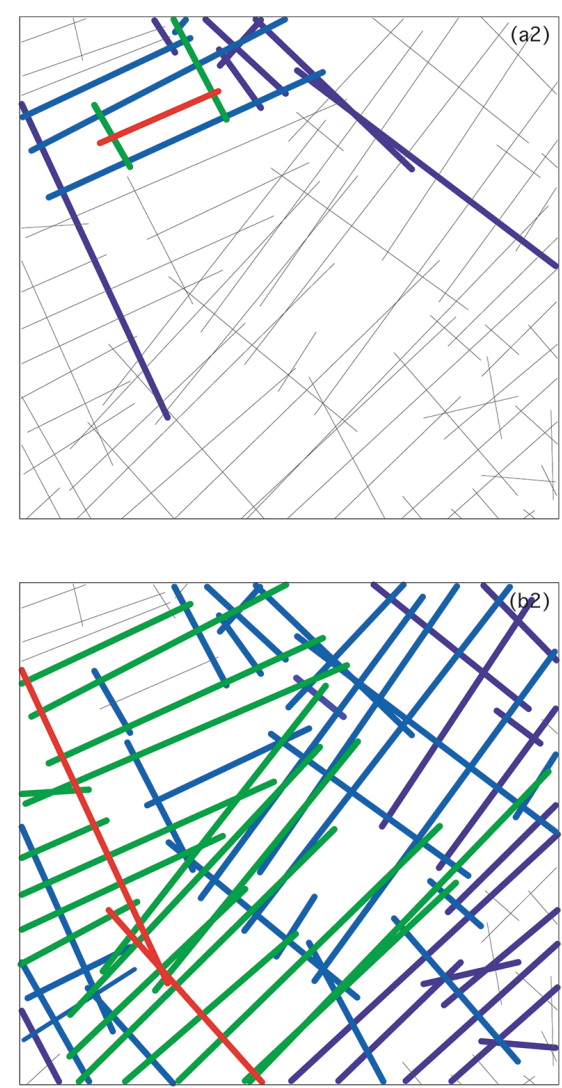

An n-step analysis, also called a point-depth analysis, illustrates how topologically deep all streets and roads are in relation to one particular street. The scheme in Fig. 2.10 illustrates an n-step analysis with six syntactic steps. For each direction change away for the street under scrutiny, the syntactic step value (step $0,1,2, \ldots \mathrm{n}$ ) increases. The length of the axial line or sightline influences the depth value, and each axial line has a single value attribute. The n-step analysis can be applied to an individual street, several streets, or a whole main route network simultaneously. N-step analyses are mostly applied to public transport stops, railway stations, and shopping streets to measure the ease of reach of these amenities from a location in the city and between them. For a public transport network, n-step analyses can measure how well the network of public transport stops covers the city in terms of direction change. This analysis can also be utilised for testing the degree of ease of reach to hospitals, schools, service centres, etc. Figure 2.11 shows an n-step analysis from all railway stations in Rotterdam. As can be seen from the figure, the areas in south-west Rotterdam have poor rail accessibility. 
Fig. 2.10 Scheme of how topological depth is calculated from a chosen street (step 0)

Fig. 2.11 N-step analysis from all railway stations in Rotterdam
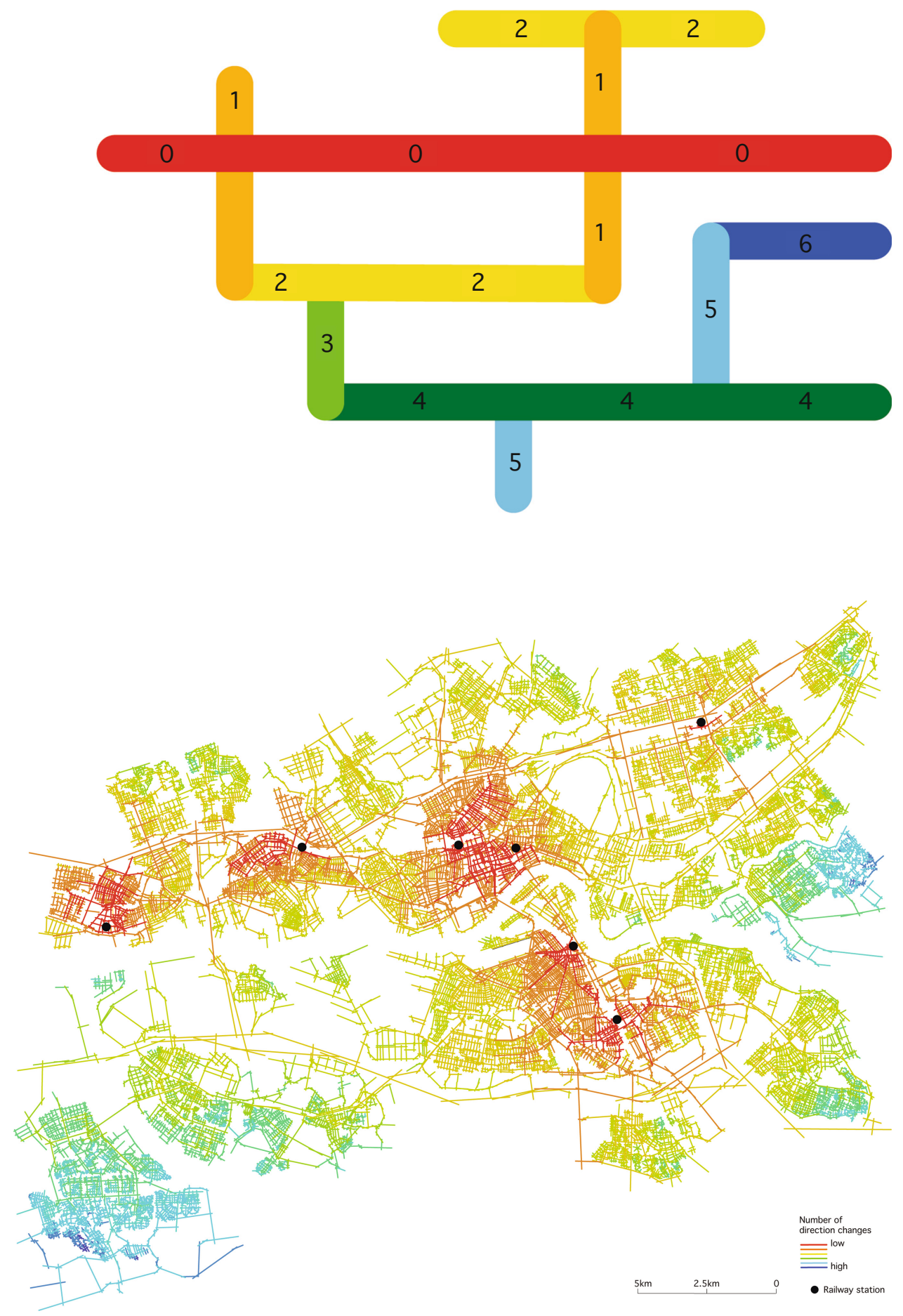


\subsection{Global Axial Integration Analysis}

'Global integration' describes how a street relates to all other streets in a predefined spatial system. This can be a neighbourhood, a quarter, a district, a village, a town, a city, or a region. In the context of globalisation, the concepts of 'global' and 'integration' are sometimes used to refer specifically to the integration of international economies through trade, foreign investment, and capital flows and to social issues such as migration, the spread of technology, and the transnational circulation of ideas, languages, and cultures (Bhagwati 2004). This must not be conflated with the meaning of the space syntax term 'global integration'. 'Global' in the space syntax jargon defines using a system-wide radius for spatial analysis. Therefore, global integration is, for example, citywide integration.

Global integration analysis estimates the degree of accessibility a street has to all other streets in the urban system taking into consideration the total number of direction changes of the urban entity (Hillier 1996). Mathematicians call this 'to-movement'. A global integration analysis calculates how spatially integrated a street axis, i.e. axial line, is relative to all other streets in the system. The fewer the changes of direction, i.e. syntactic steps, from a street to reach all locations in the system, the higher the global integration value of the street. In contrast, streets from which many direction changes have to be taken to reach all other locations in the urban system tend to have low global integration values. They are spatially segregated. In short, the longer the axial line and the higher its connectivity to other axial lines, the higher its integration value and vice versa.

Figure 2.12 shows town $\mathrm{X}$ with its morphological footprint (a), its unprocessed axial map (b), and its axial integration analysis results (c). Remember that the axial map is the set of fewest sightlines indicating movement paths. Each axial line represents a public urban space that connects to other public urban spaces. It is thus possible to calculate how each axial line is interrelated to all other axial lines in the given system. In other words, the topological depth of each axial line in relation to all other axial lines is calculated. A syntactic step is taken for each direction change. For the axial integration analysis, the values are coded in colour (c) where the red axial lines are the most integrated axes and the dark blue axial lines are the most segregated. The same colour scheme is also applied to the nodes of the justified graph (d). The justified graph illustrates how the system can be experienced from the most integrated street. Applying the logic of an axial map as the map with the fewest lines for the graph, "the lines are represented as nodes and the intersection of lines as connections between the nodes"

Fig. 2.12 Calculating total depth for town $\mathrm{X}$ with the main street $\mathrm{A}$ as the root node

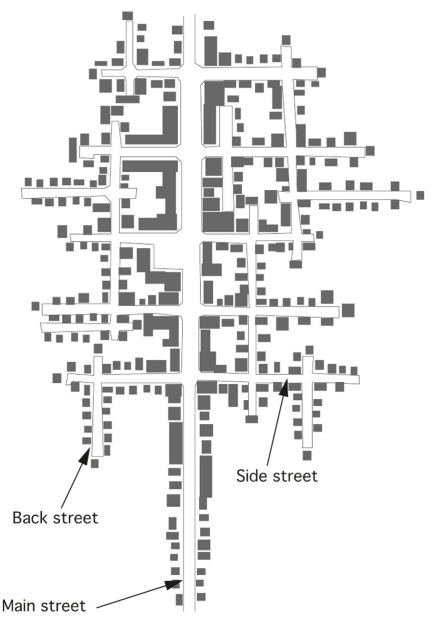

(a) Town $\mathrm{X}$

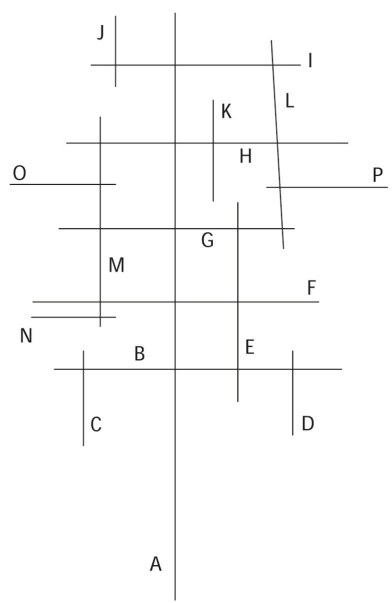

(b) Axial map

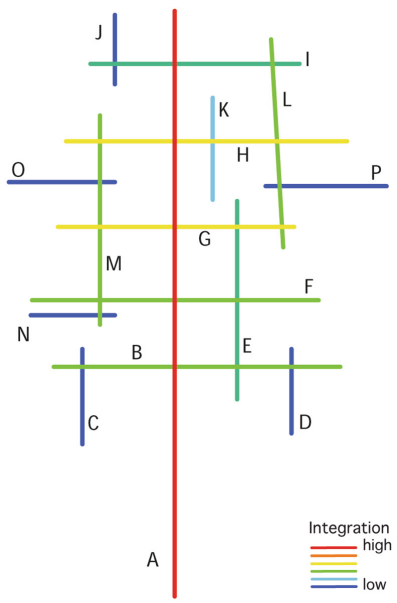

(c) Axial integration analysis

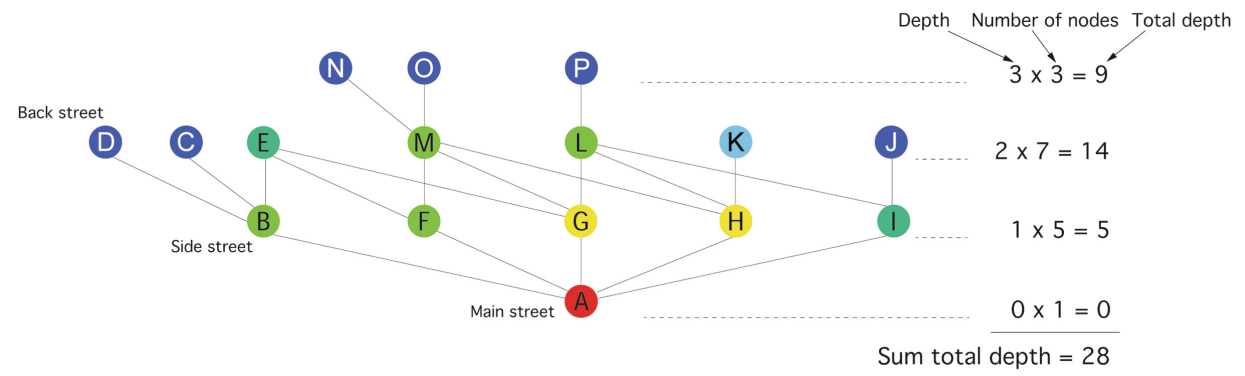

(d) j-graph and total depth calculation 
(Turner et al. 1993, p. 425). The justified graph shows how the individual components of a system are connected to each other in an abstract manner.

In the following, we show how to calculate the global axial integration for town X (Fig. 2.13). The dead-end street, indicated with a $\mathrm{C}$, is chosen as the root node or starting point or step 0. First, we calculate the total depth from the root node to all other streets. The total depth is defined by the number of spaces that must be passed through a chosen or given starting point in a system. Total depth is calculated by the sum of all possible steps from a given starting point. In other words, the total depth sum is calculated by first multiplying the number of spaces by the number of the respective topological depths and then summing up all values for each topologic depth. For our example in Fig. 2.13, the total depth is the sum of 1 root node $(0 \times 1)+$ one direction change for one space $(1 \times 1)+$ two direction changes for 3 spaces $(2 \times 3)+$ three direction changes for four spaces $(3 \times 4)+n$ direction changes for $n$ spaces.

The total depth sum of the justified graph (j-graph) in Fig. 2.13 has the numerical value of 50. From total depth, the mean depth can be derived. Mean depth is equivalent to total depth relativised to the number of axial lines or nodes of the system and represents the average number of steps needed to reach any of the axial lines in the system. In the next step, mean depth is normalised and then the effect of the system's size is relativised. This allows all urban systems to be compared with each other.

In general, the mean depth values for cities vary with different cultures. Karimi (1998, pp. 269ff) and Hillier (2001, p. 02.9) point out that the topological mean depth for most European cities is a value around 3, for US cities it is around 2, while for Arab and Persian cities it is around 5. The orthogonal grid of most postmodern North American cities is a topologically shallow system, while labyrinthine Arabic cities are known to be topologically deep systems. The reasons for these local variations of the street network are culturally driven and demonstrate how local cultural traditions influence the built form differently.

Fig. 2.13 Calculating the global integration of the cul-de-sac street C of town $\mathrm{X}$

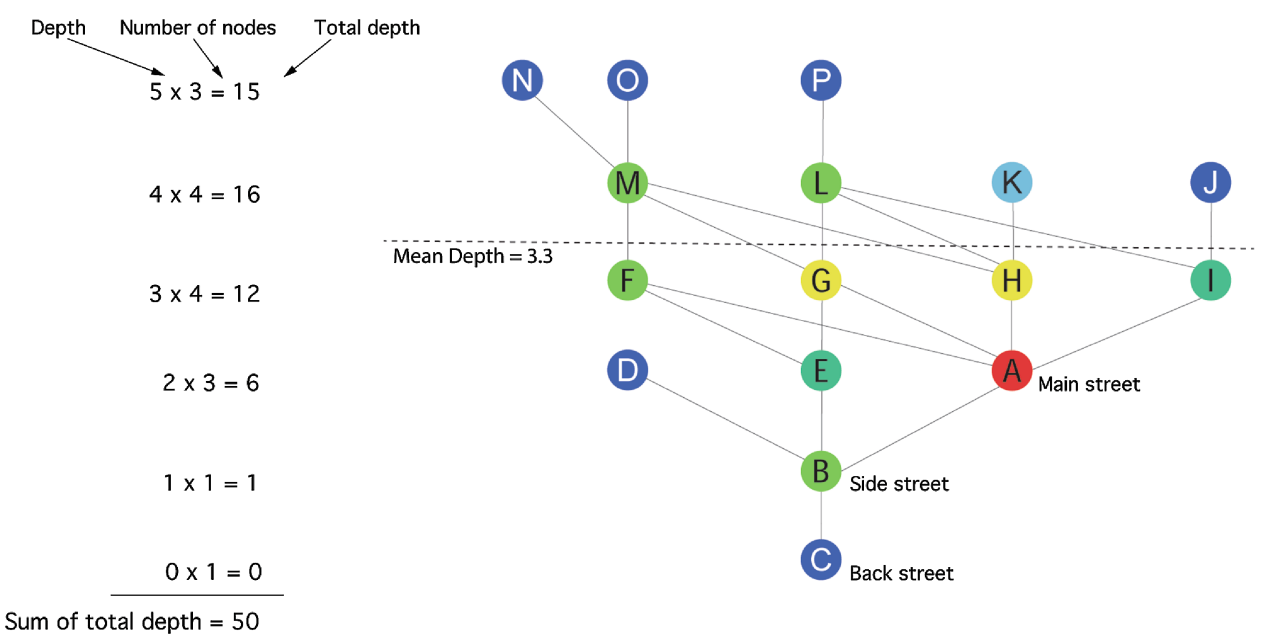

Calculating axial integration

(1) Mean depth $(M D)$

$M D=$ sum total depth $/ k-1=50 / 16-1=3.3$

(2) Real assymetry $(R A)$

$R A=2(M D-1) / k-2=2(3.3-1) / 16-2=0.33333333$

(3) Real relative assymetry (RRA)

$R R A=R A / D_{k}=0.33333333 / 0.251=1.3280212483$

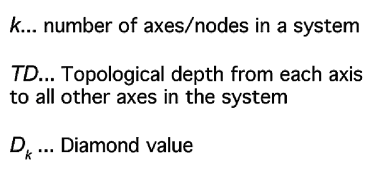

(4) Integration value of the back street

INT $=1 / R R A=1 / 1.3280212483=0.753$ 
For an in-depth understanding, and if you like mathematics, we elaborate in the next chapter how to calculate spatial integration. For the non-mathematically interested readers, we suggest moving to Sect. 2.3.2 "Global spatial integration and segregation".

\subsubsection{The Mathematical Formulas for Calculating Global Integration}

The mean depth is equivalent to the total depth relativised to the number of axial lines or nodes of the system and represents the average steps needed to reach any of the axial lines in the system. For our example, we calculate mean depth (MD) for the cul-de-sac indicated with ' $\mathrm{C}$ ' in Fig. 2.11 as

$$
M D_{C}=\frac{1}{(k-1)} \sum_{k} d_{C k}
$$

where $k$ stands for the number of axes in the system, and $d$ stands for the total depth. $\sum_{k} d_{C k}$ stands for the total depth sum from the root node $\mathrm{C}$ to all other spaces in town $\mathrm{X}$.

In addition, the relative asymmetry can be calculated. The mean depth provides the basis for the relative asymmetry, which provides a normalisation of the mean depth measure between zero and one. "This will indicate a space from which the system is shallow, that is, a space which tends to integrate the system, and high values indicate a space which tends to be segregated from the system. Relative asymmetry (or relative depth) can therefore be thought of more simply as the measure of integration" (Hillier and Hanson 1984, p. 109). Relative asymmetry is calculated as

$$
R A_{c}=\frac{1\left(M D_{C}-1\right)}{(k-2)}
$$

The interpretation of spatial integration is sensitive to the scale of the axial maps because their diamond values are dependent on the size of the spatial system at issue (Krüger and Vieira 2012, p. 194). The effect of relative asymmetry depends on the size of the system. Therefore, real relative asymmetry provides a relativisation that allows comparison of depth between different sized spatial systems. In line with Hillier and Hanson (1984, p. 110ff), "real relative asymmetry is used to eliminate the considerable effect that size can have on the level of relative asymmetry values in the real system". Real relative asymmetry is calculated as

$$
R R A_{C}=\frac{R A_{C}}{D_{k}}
$$

where $D_{k}$ stands for the diamond value that eliminates the effects that size can have on the relative asymmetry value. Adding the diamond value to the calculation of axial integration is a normalising measure of a particular node of a graph with $n$ nodes. It is a kind of 'centrality measure' that is obtained if that node were the root of a standardised graph in a diamond shape with the same number of nodes (Krüger and Vieira 2012, p. 195).

Figure 2.14 illustrates a simple experiment with a diamond-shaped graph for a particular street or node in a graph. In our example, the diamond shape $D_{k}=D_{10}$. We assume for our sample spatial system that it consists of 10 streets represented by 10 nodes in the justified graph.

The topological depth of the node at the bottom is 4 because at the broadest part of the diamond, there are four spaces. This system in Fig. 2.14 has 5 levels. The $k$ stands for the total number of spaces, and the $n$ represents the broadest part of the diamond, or the level of depth with the highest number of points. Here in this simple example the node in the equation below has the value 4 , taken from the number of spaces at the step of the broadest part of the diamond. When calculating the integration values for every single street, the $D_{k}$ value varies from street to street. 
Fig. 2.14 How to calculate diamond values

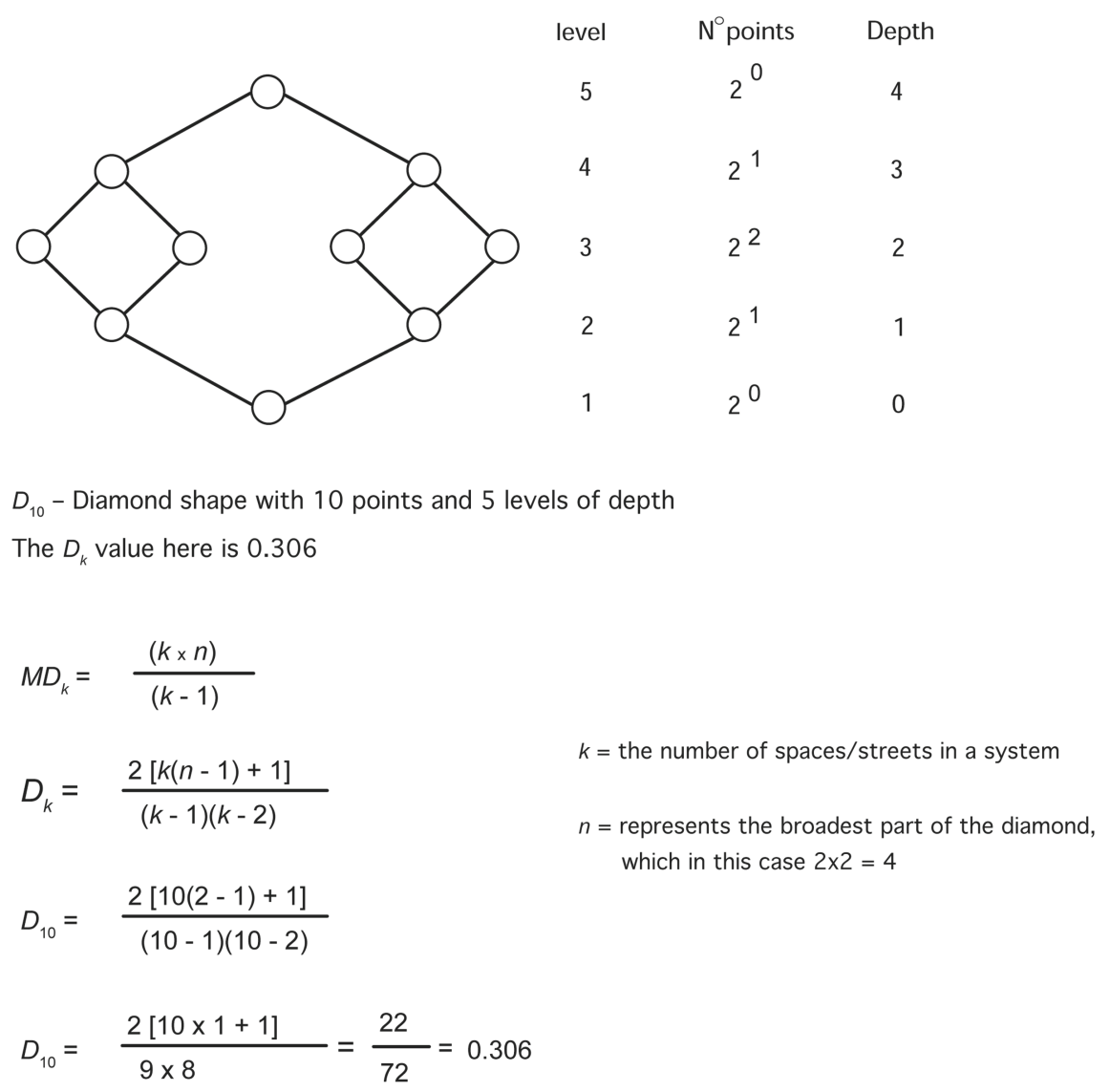

The diamond value allows one to compare the integration value of an axial line of a particular city with the integration value of an axial line in another city. Thus, spatial systems can be compared. Further, it allows one to study the changes in the integration of a street in the same city over time. Hillier and Hanson explain that the diamond value is dependent on the total number of spaces in a system (Hillier and Hanson 1984, p. 112). For example, town X comprises 16 axial lines and thus its diamond value is 0.251 given the dead-end street root node (Fig. 2.12).

High real relative asymmetry values mean greater depth, and this implies less activity and greater segregation. In order to operate with high values for integrated streets and low values for segregated streets, global integration is the reciprocal of real relative asymmetry. The global integration value is calculated as follows:

$$
\text { Global integration }=\frac{1}{R R A_{c}}
$$

Integration enables the measurement of the relative accessibility of a space within a system. Hillier and Hanson describe integration as a global measure of depth, relativised in such a way that differently sized systems can be directly compared to one another (Hillier and Hanson 1984).

A highly integrated street has a high integration value, and a highly segregated street has a low integration value. For the cul-de-sac street $\mathrm{C}$ from Fig. 2.12, the global integration value is 0.753 . This value does not tell us anything unless we compare it with other streets of the same system. Figure 2.15 shows the calculation of the main street of town X. If the total depth sum starting from the main street is calculated, we arrive at a numerical value of 28 . This means that the main street has a shorter topological distance to all other streets in comparison to the cul-de-sac street in Fig. 2.12. The global integration is almost three times higher for the main street than for the cul-de-sac street in town X, namely 2.027 compared to 0.753 . 


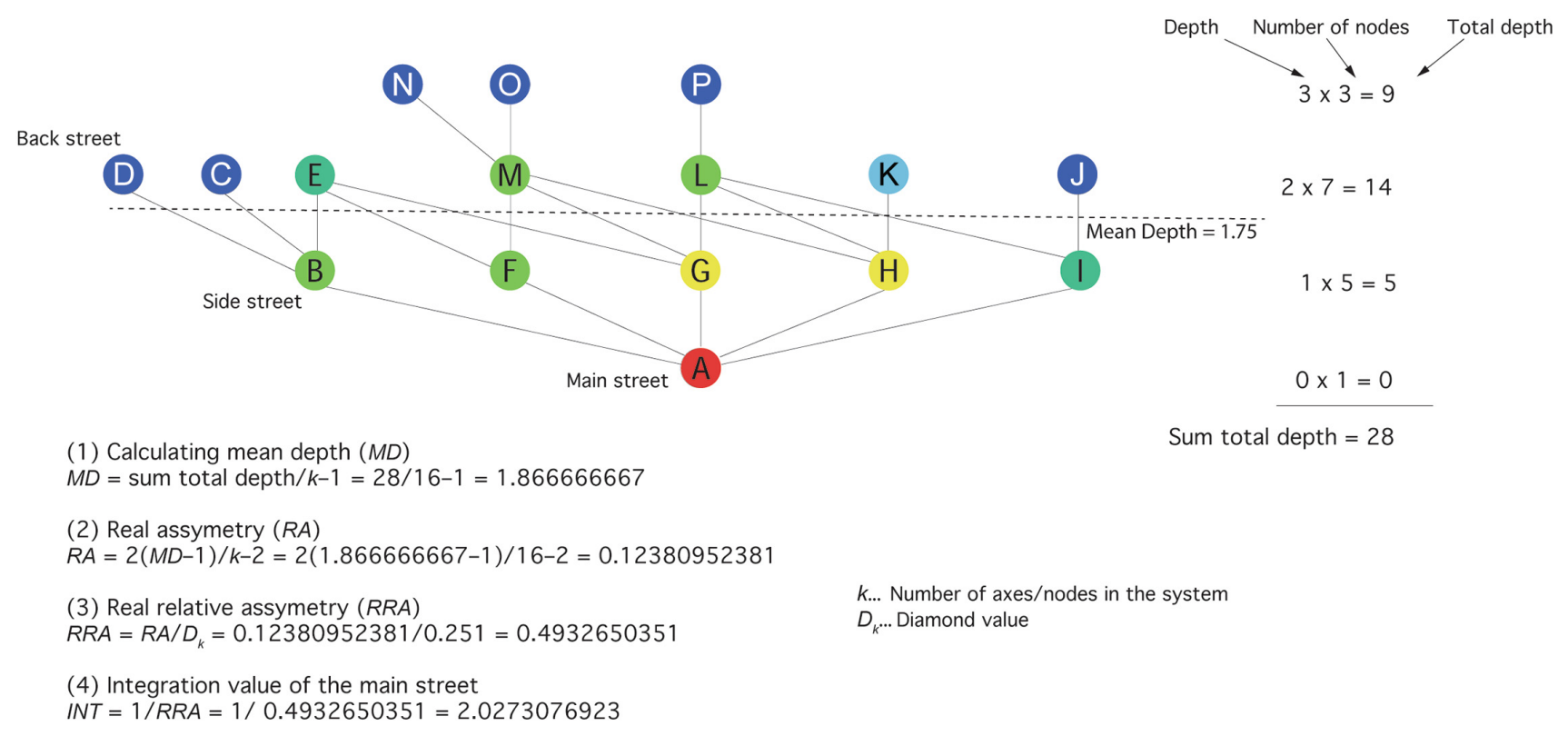

Fig. 2.15 Calculating the global integration for the main street of town $\mathrm{X}$

\subsubsection{Global Spatial Integration and Segregation}

The more integrated a street is, the shorter is its topological distance to all other streets in the urban system. Vice versa, the more segregated a street is, the longer its topological distance is to all other streets in the urban system. In Fig. 2.16, we compare the justified graphs of our example from town $\mathrm{X}$ for the dead-end street with the main street. For the justified graph with the root note 'dead-end street', the structure depicts a more tree-like structure, and for the 'main street' it takes on a more bush-like structure. The bush-shaped justified graph is topologically shallower than the tree-shaped graph. If most of the spaces are located many syntactic steps away, it is more likely that the space under scrutiny will have a low integration value. In this case, it is a segregated space, and the tree-shaped justified graph is topologically deep. For our example town $X$, all measures can be calculated manually. With the space syntax computer application Depthmap ${ }^{1}$ (Turner 2007), large cities' and regions' axial maps can be computed.

We have demonstrated how to understand integrated and segregated urban street spaces with spatial configurational relationships by applying the measure of global integration. Main urban centres have the highest global integration. Through empirical data collection, the flow of human movement and the location of various functions and activities can be registered for spaces along the axial lines and can be compared with their values of different measures (see Chap. 5).

We can apply the measure of integration to measure urban change from different time periods. In the case of Berlin, the fall of the Berlin Wall after the revolution of 9 November 1989 radically changed the street network. The erection and fall of the Berlin Wall completely changed possible movement routes from everywhere to everywhere else in the city. The wall blocked and divided the main, historic streets, thus altering pedestrian and vehicular movement in the streets of former East and West Berlin. After the fall of the Berlin Wall, the street network re-established its old configurational order with regard to the historic street network. Herein, the global integration analysis shows how the Berlin Wall affected Berlin's urban centres and individual streets by means of their spatial integration.

\footnotetext{
${ }^{1}$ A short history of computer applications for urban studies: Axman (Dalton 1988), Axwoman (Jiang et al. 2000), Meanda (Dalton 2001), Mindwalk (Figueiredo and Amorim 2005), Place Syntax for QGIS (Ståhle et al. 2005), Depthmap (Turner 2007) Urban Network Analysis Toolbox for ArcGIS (Sevtsuk 2010), Space Syntax application for QGIS (Gil et al. 2015) and Form Syntax as a plug in for GIS (Ye and van Nes 2016) for a synthesis.
} 


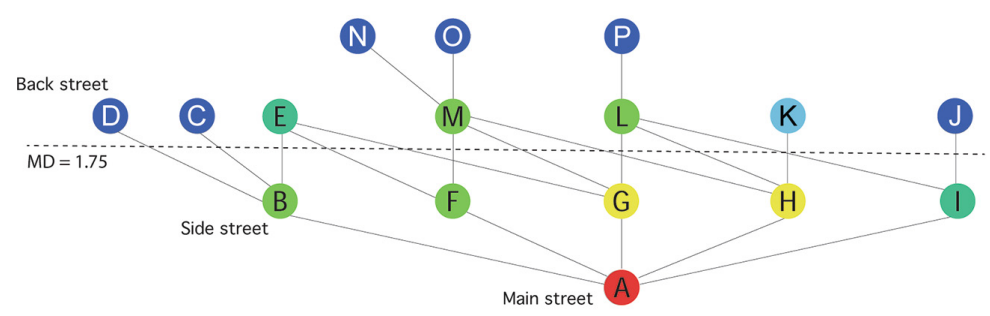

The main street $\mathrm{A}$ is the most integrated street. Total depth $=28$

Integration value $=2.0273076923$

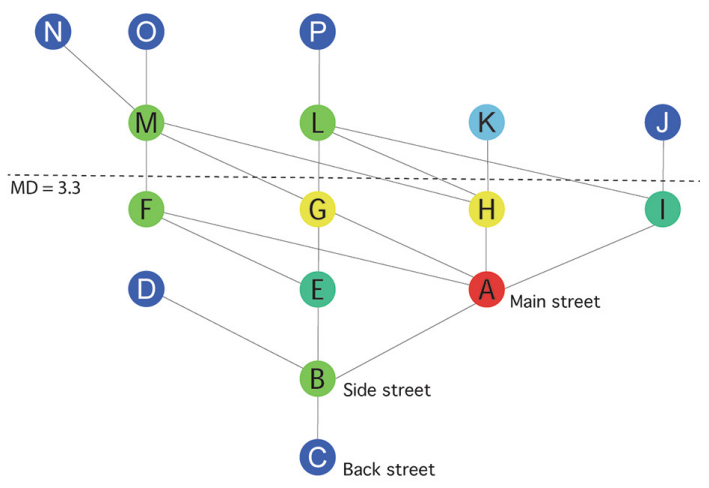

The back street $\mathrm{C}$ is the most segregated street. Total depth $=50$ Integration value $=0.753$

Fig. 2.16 Comparison of two justified graphs with the root nodes of a dead-end street and the main street

Figure 2.17 illustrates the urban change of Berlin by applying the measure of integration in 1988 and 2005. The 1988 integration analysis depicts Kurfürstendamm and Tauentzienstraße as the most integrated streets in West Berlin and Alexanderplatz as the most integrated for East Berlin. Alexanderplatz is located at the intersection of the highly integrated streets Frankfurter Allee and Greifswalder Straße, and this was the urban centre for East Berlin. Kreuzberg and Wedding are segregated areas in the analysis, indicated by green and blue axial lines, suffering from the isolated position caused by the Berlin Wall in West Berlin. The integration analysis of reunified Berlin in 2005 reveals that the central urban core has shifted to Friedrichstraße. Friedrichstraße and Potsdamer Platz were the most integrated areas of unified Berlin as far back as the 1930s, but during the era of the wall they were segregated areas. The globally, i.e. citywide, integrated Friedrichstraße is currently Berlin's high street, comparable to Oxford Street in London.

Active land use like shops is sensitive to shifting centralities, and Berlin is clearly an example of this. Friedrichstraße and Potsdamer Platz are currently Berlin's urban main centres, and the former urban main centres of Alexanderplatz and Kurfürstendamm have lost their highly central position in the urban system since the fall of the wall. Accordingly, the two centres around Alexanderplatz and Kurfürstendamm flourished during the period of the wall, while the other two centres around Friedrichstraße and Potsdamer Platz faded away during this period and flourished again after the wall came down. At present, Kurfürstendamm is a high-end luxury shopping street. Interestingly, luxury brands are often located in streets with high connectivity to their vicinities. These luxury brands avoid streets with the highest integration values, with the purpose of reducing the flow of random customers from mainstream society. Most of the mainstream customers just come and look, but cannot afford to buy. Desyllas (2000) investigated how land values changed in Berlin before and after 9 November 1989, and he showed that real estate prices increased in areas where the integration values of the street network increased after the fall of the wall. Figure 2.18 shows impressions from Berlin.

The Berlin example showcases the twofold challenge with global integration analysis. First, the urban fringe and the suburbs are highlighted as very segregated areas close to the system's boundary, which is referred to in the analysis as the so-called 'edge effect'. This will be explained in the next section. Second, many cities consist of several centres, ranging from the city's main centre to local neighbourhood shopping streets. Global integration analysis highlights the city's main centre whether the centre is a car-based shopping mall or a pedestrian-friendly high street with adjacent streets. Local sub-centres tend to be poorly highlighted in a global integration analysis. 


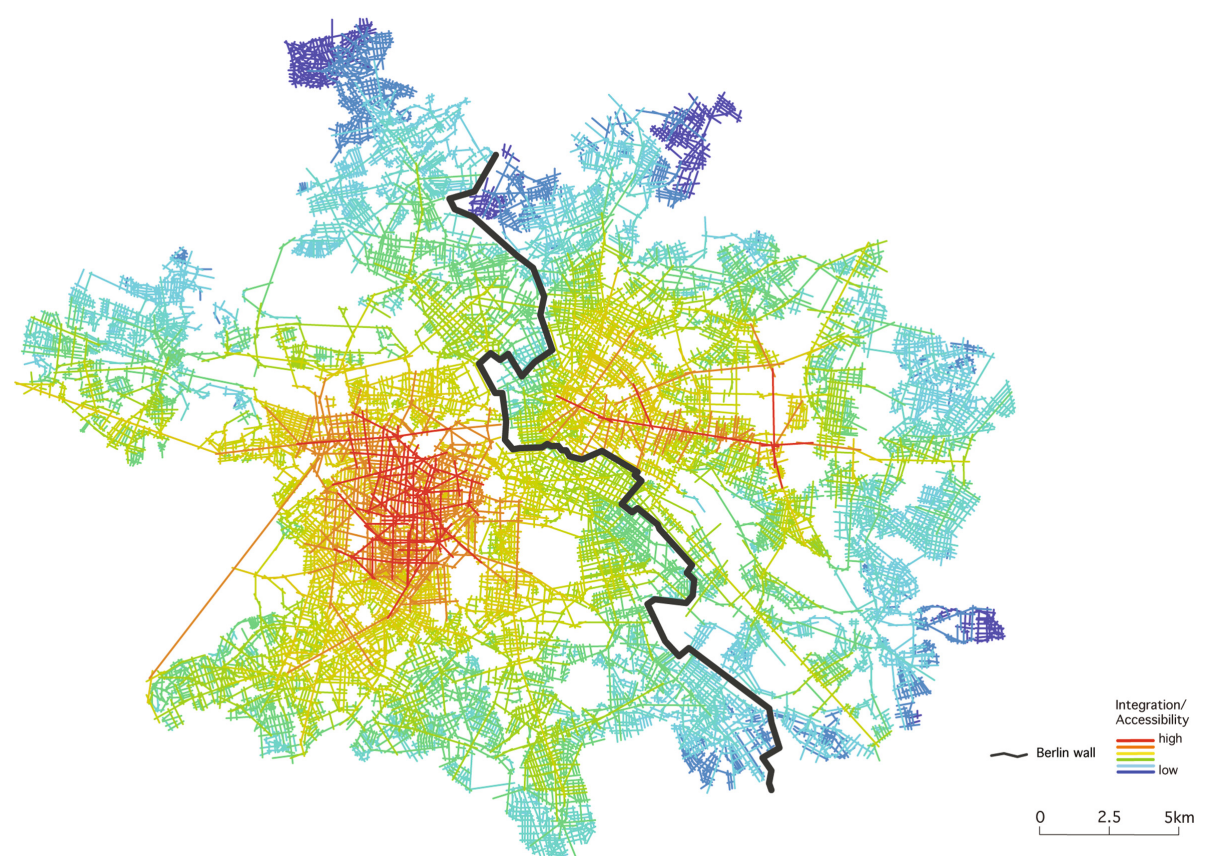

(a) Global (citywide) axial integration analysis of Berlin in 1988

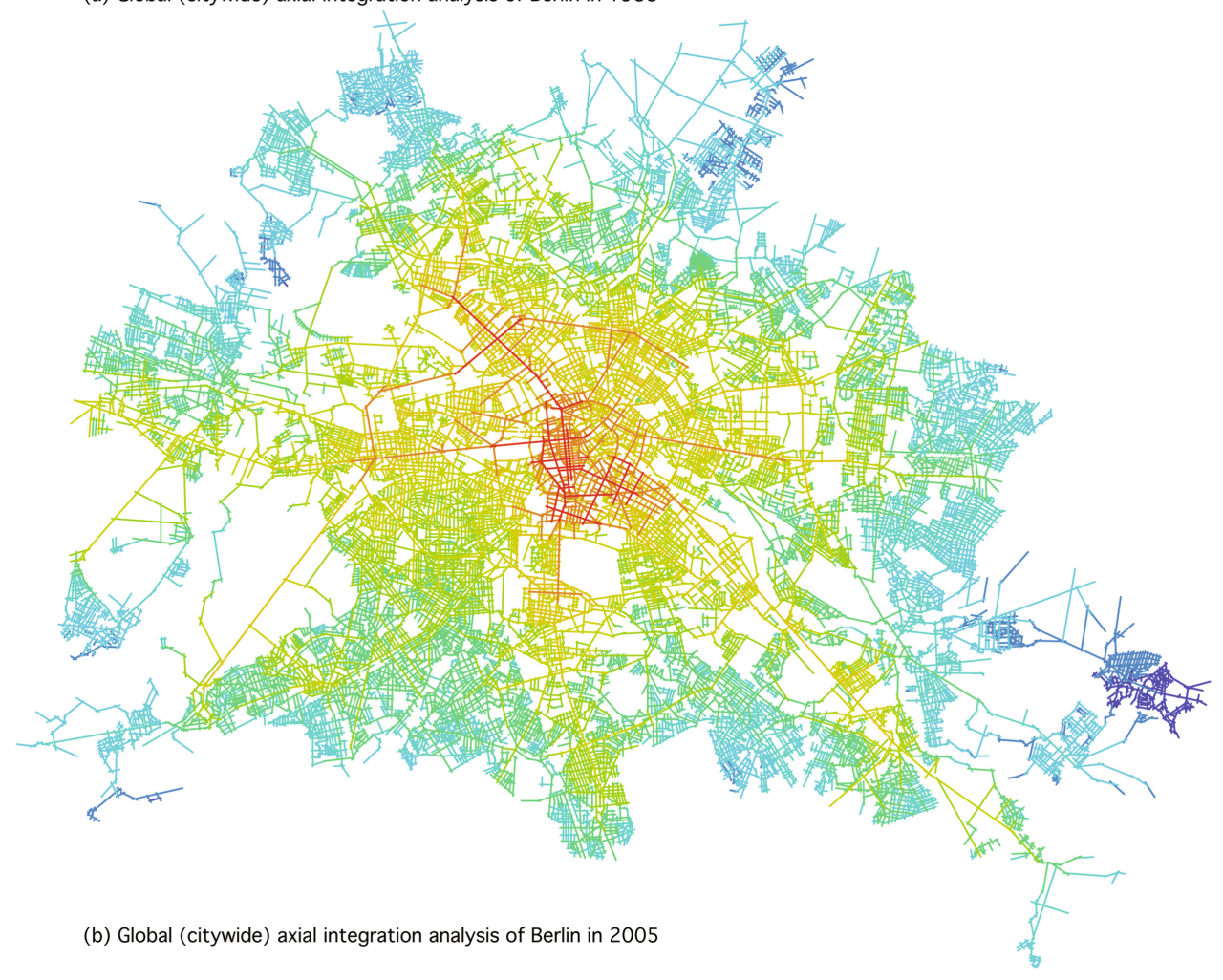

Fig. 2.17 Global integration analysis of Berlin in the area of the Berlin Wall in 1988 (a) and in reunified Berlin in 2005 (b) 
Fig. 2.18 Impressions from Berlin: Friedrichstraße, Alexanderplatz, Potsdamer Platz, Brandenburger Tor, and the Berlin Wall
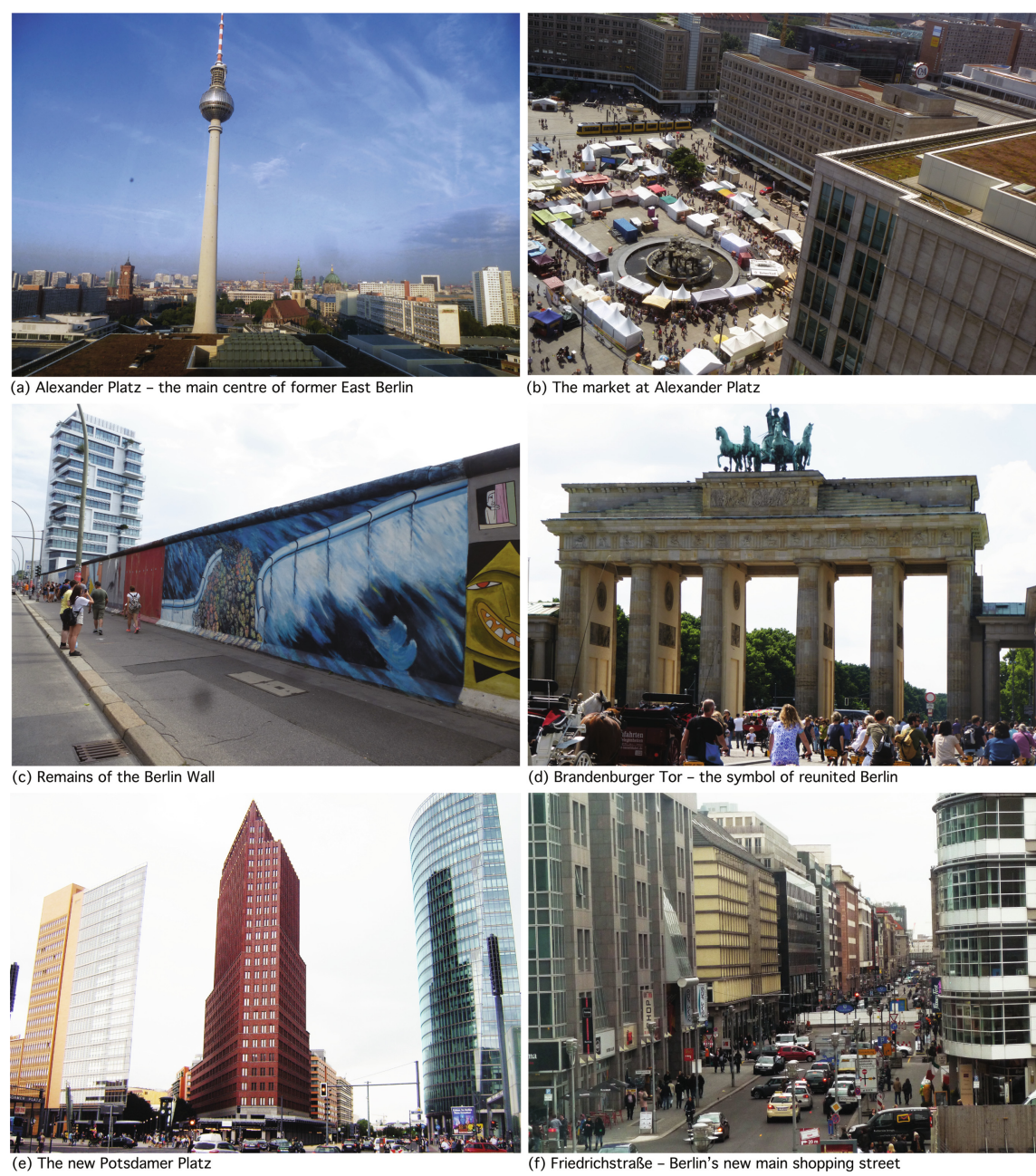

(f) Friedrichstraße - Berlin's new main shopping street

\subsection{Local Integration Analysis}

Many urban neighbourhoods and suburbs have their own local shopping areas. These local urban centres are poorly highlighted in a global integration analysis, and local urban shopping areas often have low global integration values but high local integration values. There are two options for measuring the local integration of these centres: (a) a global integration analysis of only the urban centre under scrutiny, and (b) a local integration analysis of the entire city. This section will examine the latter in detail.

In general, the key to assessing local integration is in calculating the average mean depth value of all streets within a certain syntactic radius, for example, a radius of three. For the topological radius, integral numbers have to be chosen because the radius number represents the number of syntactic steps - one cannot take a fractional topological or syntactic step like 2.5. For example, two syntactic steps include one direction change including the starting point (root node) for a radius of two. Three syntactic steps are two direction changes including the starting point for a radius of three. For a radius of four, all streets beyond three topological steps away from the street representing the root node have to be excluded. 
Town X has a low average mean value of mean depth 2.525 for the whole system due to its orthogonal street network and is thus a topologically shallow system. Figure 2.19 illustrates the local integration radius of three for town $\mathrm{X}$. For our example (a), we chose the main street as the root node as indicated in red. Thus, the axial lines indicated with an $\mathrm{N}$, $\mathrm{O}$, and $\mathrm{P}$ are not part of the integration radius three analysis. In example (b), we considered the cul-de-sac as the root node as indicated in red. For the integration radius three analysis, only five axial lines are considered. Figure 2.19 (c) depicts a local integration radius three analysis for the entire town $\mathrm{X}$. Thus, for each axial line, the integration radius three values are calculated. When comparing the local integration values of all streets, street $\mathrm{M}$ has the highest local integration value followed by the main street for town X (Fig. 2.20).

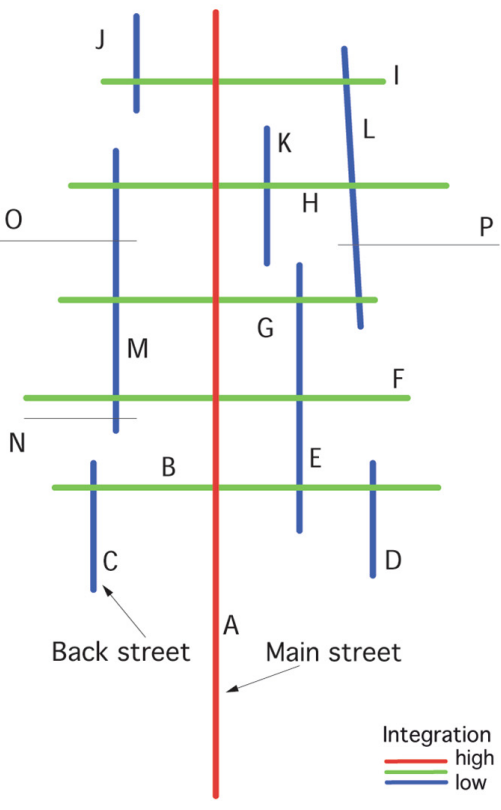

(a) Topological radius $=3$ analysis, computed from the main street

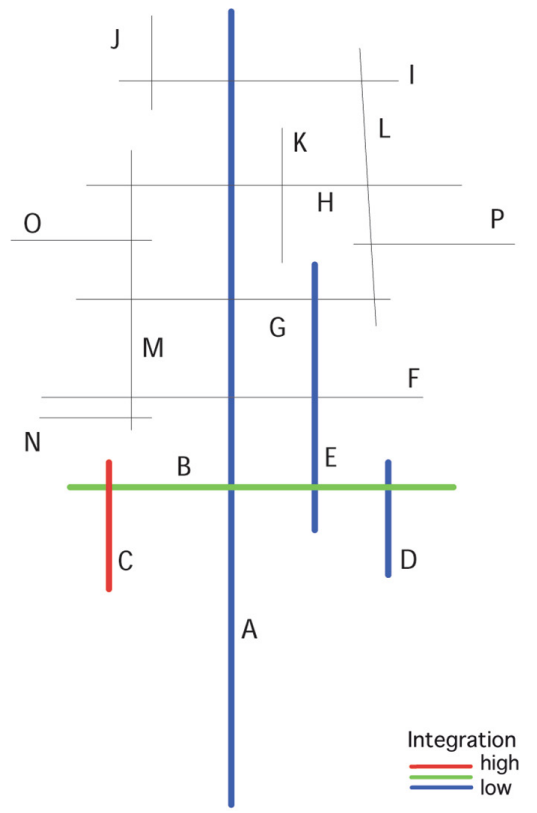

(b) Topological radius $=3$ analysis, computed from the back street

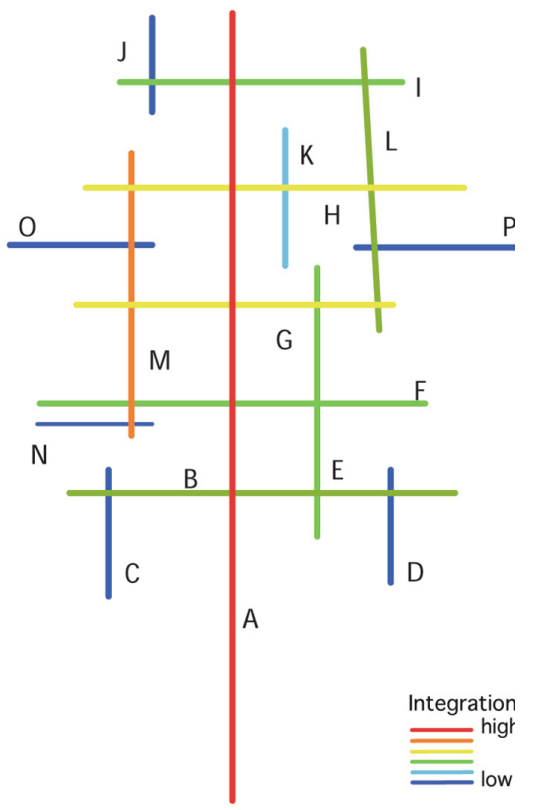

(c) Local integration radius $=3$ analysis computed from every street

Fig. 2.19 The principle of local integration

In Fig. 2.20, we show a comparison of how global and local integration is calculated for town $\mathrm{X}$ with street $\mathrm{M}$ as the root node. In the global integration analysis, the sum of the total depth is 34 , while for the local integration analysis it is 13 . None of the urban spaces beyond the second syntactic step from the street for which the local integration is calculated are taken into account. This is also highlighted in the justified graph. Global and local integration analyses differ from each other by the total depth sum value and the diamond value in calculating the real relative asymmetry. Due to the lower number of spaces taken into consideration for the local integration analysis, the diamond value differs for the entire system. A description of how and why the diamond value is calculated is explained in Sect. 2.3.1. For street M, the diamond value is 0.267 .

In Fig. 2.21, we show justified graphs with the main street and cul-de-sac street as root nodes. The local integration value for the main street A is 2.6022857143, and for the dead-end street C it is 0.7039999998 . Street M's local integration value is 2.754 (Fig. 2.20). The main street has a lower local integration value compared to street $\mathrm{M}$, which means that street $\mathrm{M}$ is locally more highly integrated than the main street.

Global and local integration allows one to measure the spatial impact for an entire city before and after urban interventions. Hillier (1998) and Hillier et al. (1998) state that pedestrian flow rates through cities correlate with local integration values, while vehicle flow rates correspond with global integration values. Furthermore, a local integration analysis indicates the local shopping areas in a city. In order to illustrate the differences between local and city scale centres, we use the Norwegian capital of Oslo as a case. 


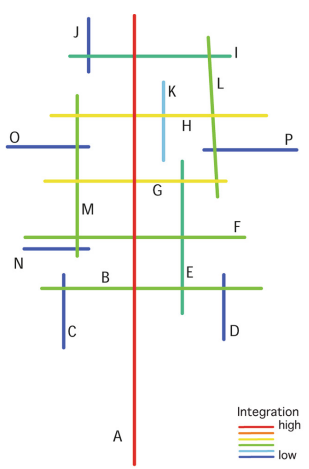

(a) Global axial integration analysis, radius $=n$

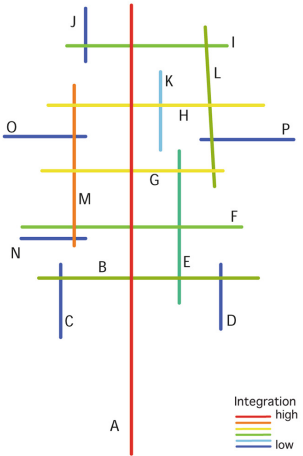

(b) Local axial integration analysis, radius $=3$

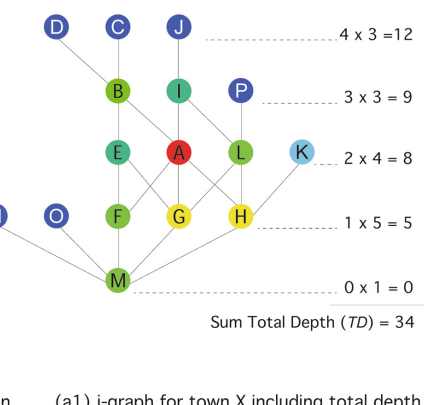

(a1) j-graph for town $\mathrm{X}$ including total depth

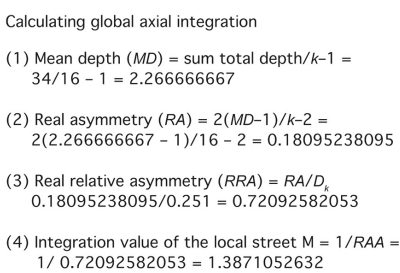

(a1) Calculation of global axial integration

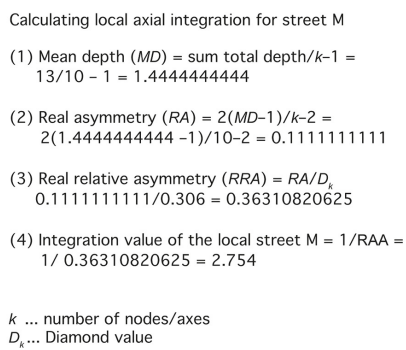

Calculating local axial integration for street $M$ (1) Mean depth $(M D)=$ sum total depth $/ k-1=$ $13 / 10-1=1.4444444444$

(2) Real asymmetry $(R A)=2(M D-1) / k-2=$ $2(1.4444444444-1) / 10-2=0.1111111111$

(3) Real relative asymmetry $(R R A)=R A / D_{k}$ $0.1111111111 / 0.306=0.36310820625$

(4) Integration value of the local street $M=1 /$ RAA $=$ $1 / 0.36310820625=2.754$

$k \ldots$ number of nodes $/$ axes
$D_{k} \ldots$ Diamond value

Sum Total Depth $(T D)=13$

(b2) Calculation of local axial integration

Fig. 2.20 Calculating the global (a) and local (b) integration of street $\mathrm{M}$ for town $\mathrm{X}$

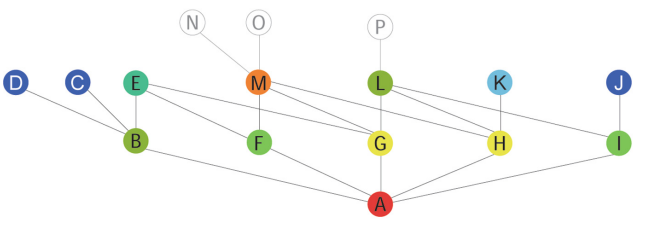

Sum Total Depth $(T D)=19$

(a) j-graph and local axial integration calculation for the main street A
Calculating local axial integration for the main street A

(1) Mean depth $(M D)=$ sum total depth $/ k-1=$ $19 / 13-1=1.583333333$

(2) Real asymmetry $(R A)=2(M D-1) / k-2=$ $2(1.583333333-1) / 13-2=0.10606060606$

(3) Real relative asymmetry $(R R A)=R A / D$ $0.10606060606 / 0.276=0.38427755819$

(4) Integration value of the main street $A\left(I N T_{A}\right)=1 / R A A=$ $1 / 0.38427755819=2.6022857143$

$$
\begin{aligned}
& k \ldots \text { number of nodes/axes } \\
& D_{k} \ldots \text { Diamond value }
\end{aligned}
$$

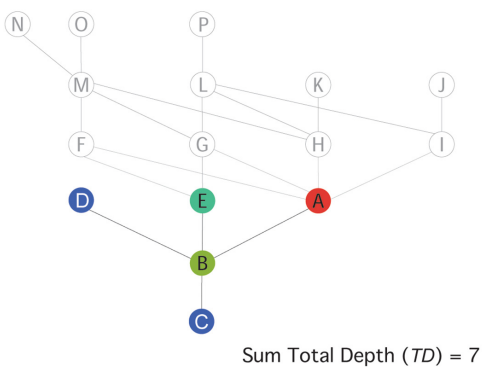

Calculating local axial integration for the back street $C$

(1) Mean depth $(M D)=$ sum total depth $/ k-1=$ $7 / 5-1=1.75$

(2) Real asymmetry $(R A)=2(M D-1) / k-2=$ $2(1.75-1) / 5-2=0.5$

(3) Real relative asymmetry $(R R A)=R A / D$ $0.5 / 0.352=1.4204545455$

(4) Integration value of the back street $C\left(I N T_{C}\right)=1 / R A A=$ $1 / 1.4204545455=0.7039999998$

(b) j-graph and local axial integration calculation for the back street $\mathrm{C}$

Fig. 2.21 Calculating local integration for the main street (a) and dead-end (cul-de-sac) street (b) of town X 
Figure 2.22 shows a global (a) and local (b) integration analyses of Oslo. The most globally integrated axial lines are the outer ring road followed by the middle ring road. Both ring roads serve vehicular movement. In particular, the outer ring road is a busy car-based road. At every junction, a car-based shopping mall is located. The locally highly integrated streets are Oslo's most pedestrian-friendly and vital shopping streets, and these streets have mixed land use with individual small shops, cafés, and chain stores.
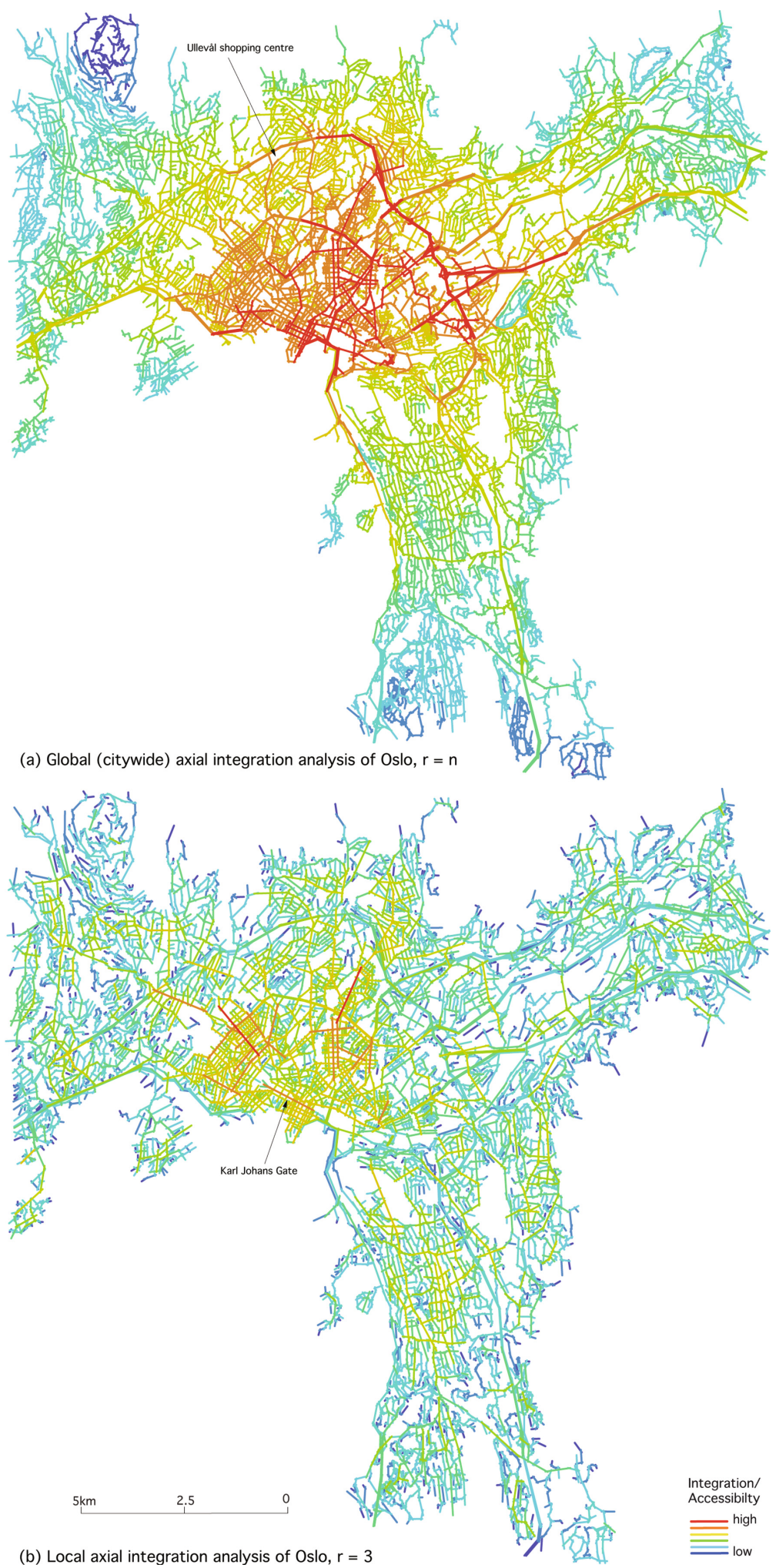

Fig. 2.22 Global (a) and local (b) integration analyses of Oslo 
Oslo is known to be a compact city in comparison to other Norwegian cities. Due to a strict policy to protect agricultural land, Norwegian settlements tend to be scattered with batches of agricultural land in between. In Oslo, the Ullevål shopping centre was built in the 1980s after ring road 3 was finalised. Originally, there were no intentions to give planning permission for such a shopping centre. However, the market forces were stronger than the planning system, and currently car-based shopping centres are located at every junction of Oslo's globally integrated ring road 3. The locally integrated Karl Johans Gate is Oslo's pedestrianised main shopping street. This linear shopping street is well connected to its direct vicinity (Fig. 2.23).
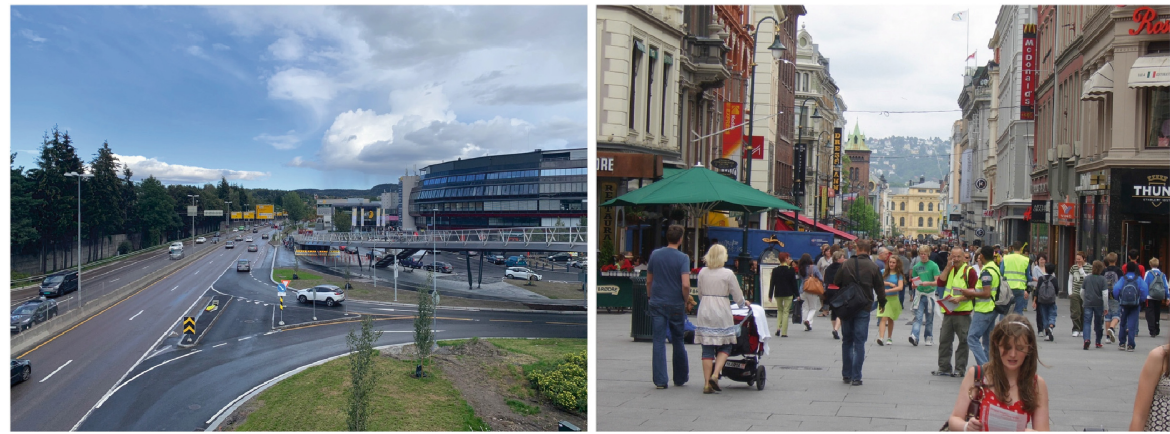

Fig. 2.23 The Ullevål shopping centre is located adjacent to a highly integrated car-based ring road (left), and Karl Johans Gate in Oslo as an example of a locally well-integrated shopping street in Oslo (right)

Where the topography is hilly and streets are curvy, local shopping areas are poorly highlighted in the global and local integration analysis. This is because a curvy street is modelled with several shorter axial lines. Thus, to provide a solution for this challenge, we here introduce angular segment analysis.

\subsection{Angular Segment Analysis}

What angular segment analysis adds to integration analysis is that each street segment is weighted by the angle of its connection to other street segments. Each axial line is split into a number of street segments. The split of an axial line takes place at every junction, and the relationships between junctions in a street network are now taken into account.

The difference between axial and segment analyses is as follows. In the axial analysis, a curved street is modelled with several axial lines. In the angular segment analysis, a curve is modelled with several street segments derived from the axial line that snap to each other at the segment ends. Curves that are modelled with a number of street segments are processed and analysed as one spatial entity. When two or more axial lines intersect, it is defined as a junction. In the axial line representation of a curved street, a 'junction' occurs every time there is a change of direction.

However, the angular segment analysis quantifies how likely the selected street segment is to be part of a trip for all possible combinations of origin and destination in an urban system (Turner 2001). Angular segment analysis is further coined 'choice', 'angular choice', and 'potential through movement'. The empirical support for this analysis was initiated by the research of Ruth Conroy Dalton with her British Library hypothesis, and she showed how angles influence people's choices for the simplest route between their origin and destination. Conroy Dalton concluded that "people tend to conserve linearity through their routes with minimal angle deviation" (Dalton 2001, p. 47.11).

This means that for urban public spaces, the angular relationships between streets play a role in the way people orientate and navigate themselves in and through the built environment. When changing direction, people tend to choose routes where the angles between different streets and street junctions are close to $90^{\circ}$ or $180^{\circ}$. Urban blocks with rare angles like 30 and $60^{\circ}$ are presumably difficult for orientation, and people tend to get lost. As mentioned earlier, Figuradeiro's concept of continuous lines (2005) has been one step towards adding angular weighting into space syntax. Defining streets that deviate less than $35^{\circ}$ from each other as one continuous line contributes to the identification of the main routes through the city. 
Moreover, Conroy Dalton discovered that people tend to choose the longest street with the shortest angle towards their aimed direction. In other words, people choose the straightest possible route to avoid the complexity of wayfinding through urban street grids (Dalton 2001). From an urbanism perspective, the same tendencies can be identified in cities as in Conroy Dalton's British Library example. Figure 2.24 shows a map from Delft with two different routes between home and work for one of the authors' previous home addresses. The longest street with the lowest angular deviation from the destination is chosen at the beginning of the journey. It is only at the end of the journey that the route choice becomes slightly fragmented on account of a bridge over a canal. In general, the longest route with the lowest angular deviation wins over the shortest route with a larger angular deviation (Fig. 2.24).

Fig. 2.24 Bicycle route from home to work in Delft

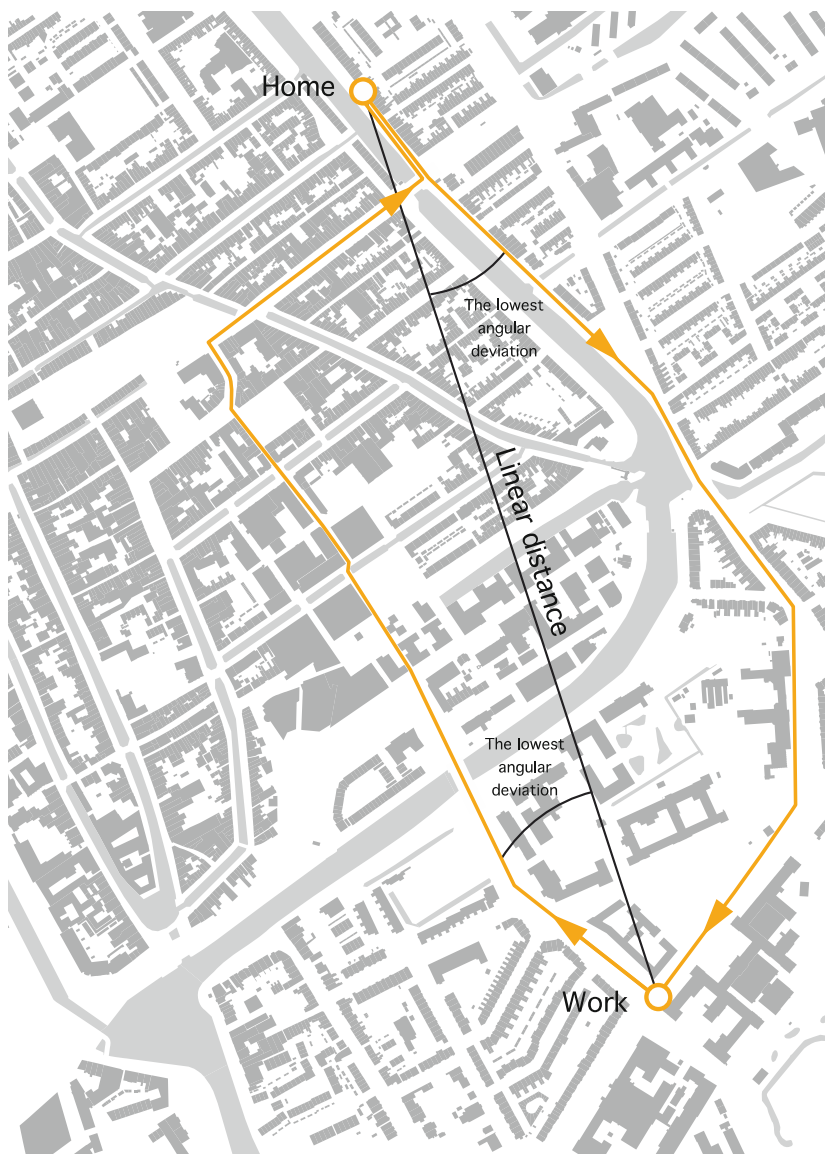


Fig. 2.25 Angular weighting of street segments (redrawn; original: Dalton 2005)

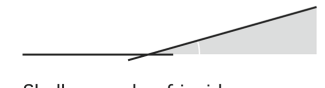

Shallow angle of incidence

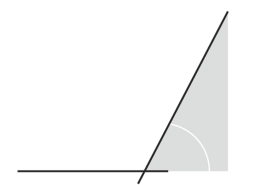

Sharp angle of incidence

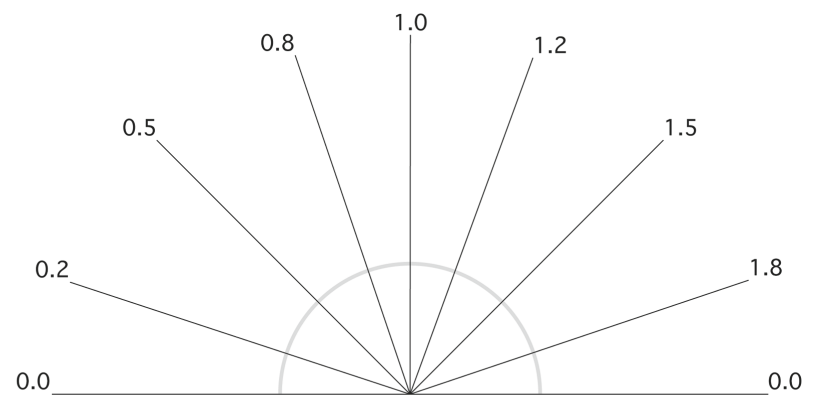

Angular weighting used in Depthmap (Dalton, 2005)

Figure 2.25 illustrates the angular weighting used in the computer application Depthmap. Shallow angles of incidence of nearly $180^{\circ}$ are weighted with the numerical value zero, and sharp angles close to $90^{\circ}$ are weighted with a numerical value of one.

Integrating angles into the calculation of street systems is based on two major factors. First, orientation in built environments is easier for people when the street network is not dominated by 'strange' angles. Second, people linearise routes when taking shallower turns towards their goals. For angles between street segments in cities, people tend to round angles to $90^{\circ}$. The subject memory of turns is better for right angles, thus when there is a doubt, a turn is rounded to $90^{\circ}$ for better placement of oneself for choosing a route for a trip in the urban network (Turner 2001). In general, there are three types of turns for people with regard to cities: no turn, fork, and right angle (Dalton 2001).

In general, segment analysis is about the absolute change of direction (Turner 2000) when moving from origin A to destination B. The basic underlying idea for angular segment analysis is the minimal change of direction. The distinction is between minimum angular path (MAP) (Turner 2000) and the minimum distance path (MDP) as a Euclidean measure between two locations in the urban system. A tourist will mostly follow the minimum angular path, and a local will mostly follow the shortest Euclidean distance.

The major impact of angular segment analysis is that it allows one to use the way people orientate and navigate themselves to visualise the potential choice of routes in a network. This includes people's cognitive assumptions regarding the network in terms of angles or linearising routes. In Fig. 2.26, we illustrate, based on a simple example, two different routes between two locations in the network. The dotted line route has the highest number of angular deviations, but the shorter Euclidean distance between both locations. Conversely, the solid line has the fewest angular deviations, but the longer Euclidean distance between both locations.

Fig. 2.26 The Manhattan gridminimum angular path versus Euclidean path (redrawn; original: Turner 2000, p. 4)

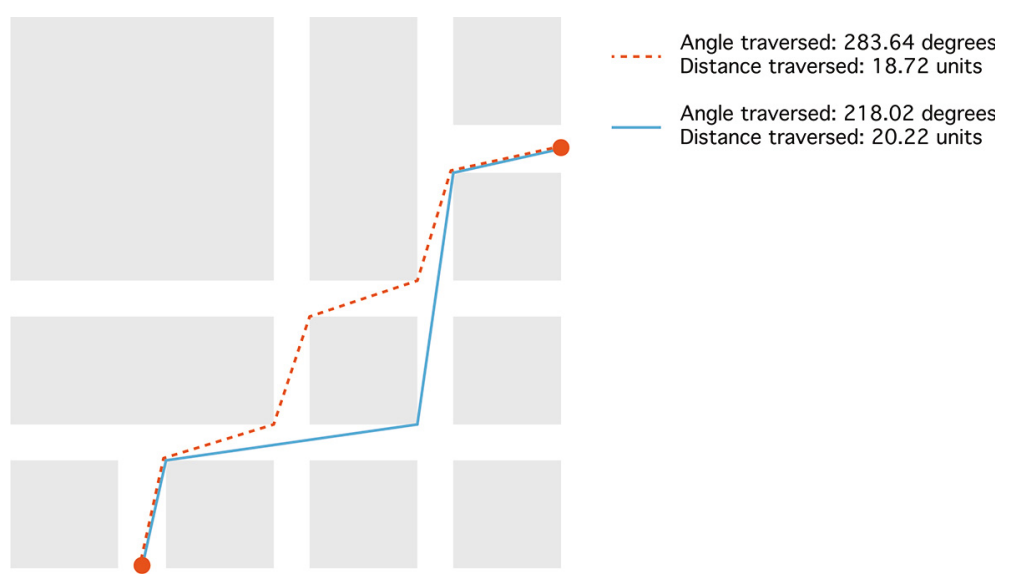


For the operationalisation of angular segment analysis, the axial lines are broken into (axial) segments. Figure 2.27 shows three different basic spatial representations of the street and road networks for a particular case (a). In the axial representation (b), the given case consists of four axes. When breaking the axial map into a segment map, the case consists of six segments (c).

Recently, for analysing large metropolitan areas, whole regions, or even whole countries, the road centre line map is applied. Herein, Turner (2007) has demonstrated the applicability of road centre line maps in addition to axial maps for segment analysis. Drawing an axial map is time-consuming. Using a road centre line file can save a lot of time, but only if all road centre lines are well connected, there are not several road centre lines stacked on top of each other, and all roads, streets, and paths are indicated. Cleaning up and complementing a road centre line file can be very time consuming. However, which approach to use depends on your research or project focus. When you are working on a metropolitan or regional scale, then the case-based routes are easy to aggregate from the road centre line. Sometimes a so-called 'hybrid model', a combination of road centre lines and an axial map, is used. When working on a small town or neighbourhood level, the time used for making a hand-drawn axial map can be the same as the time used for cleaning up a segment map aggregated from the road centre line. Moreover, the hand-drawn axial map contributes to greater accuracy than the road centre line map.

Fig. 2.27 The various spatial representations of the street and road network

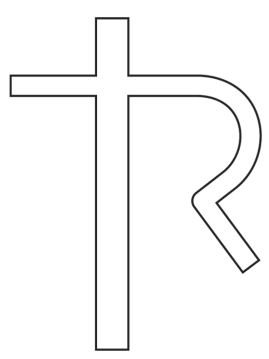

(a) The street and road network

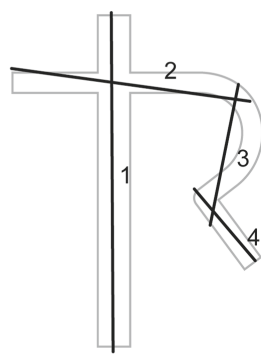

(b) Axial representation (Hillier and Hanson, 1984)

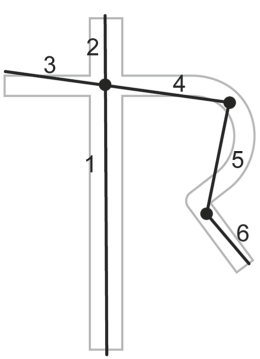

(c) Segment representation (Hillier and lida, 2005)

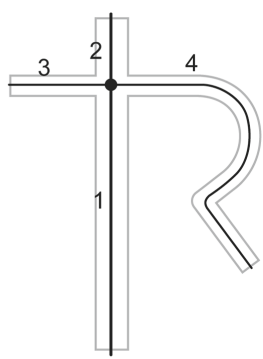

(d) Road/path center line (Turner, 2005)

Here in our case, the road or path centre line consists of four segments (Fig. 2.27d). The continuous line nr. 4 thus has both a length and a curvature. In the case of the road centre line map, the segment is represented as one segment between two junctions (Bruyns et al. 2019). Turner verified (2007, p. 533) "that the road-centre line maps and space syntax axial line maps may be analysed in comparable fashion when using angular segment analysis". For the objectivity of the spatial analyses, the segment length thus has to be taken into consideration when processing the road centre line map. The same spatial structure is shown when breaking road centre lines maps or axial maps into segment maps and when segment length is used for the analysis (Turner 2007).

For angular segment analysis, the total segment depth is calculated "by the sum of the angle turns from the starting segment to any other segment within the urban system" (Turner 2005, p. 145). In Sect. 2.2, we used only topological steps in the one-, two-, three-, and n-step analyses for visualising topological step depths. For every direction change away from a line, a topological step is taken. Now we add the angular weighting between the lines into the step-depth analysis. The sharper the angular turn from a segment, the higher the step is weighted.

Figure 2.28 illustrates how various types of step depths (or direction changes) are calculated from a particular street segment or axial line for an organic street pattern (a) with a radius n. Example (b) shows an axial step depth starting from the red street axis, example (c) shows a segment step depth from the red street segment, and example (d) shows an angular-weighted step depth analysis from the red street segment with a black frame.

For the axial depth calculation, the axial map is the basis. As mentioned earlier, the axial map represents the longest sightlines indicating paths, and each direction change is one topological step. This refers to the axial topological depth calculation (see Fig. 2.10). The segment depth calculation considers every junction at which a syntactic step is taken. Here in Fig. 2.28, we see that the red axial from example (b) is split into two street segments due to a junction (c). For the angular-weighted step depth calculation in example (d), it is important to know that the closer the connecting street segment to the previous street segment is to $180^{\circ}$, the closer the numerical weight value is to 0 . Street segments with a zero degree of angular deviation from the red segment in example (d) have zero values in the angular step depth analyses. Therefore, these segments are coloured in red. An angle of $90^{\circ}$ has the numerical weight value of 1 and is in our example coloured in yellow. A sharp angle of $40^{\circ}$ has a weighted value of 1.6 and is indicated in light green. For an explanation of the angular weighting, see Fig. 2.25. The colour codes allow for visualisation and comparison of different step depths. 
Fig. 2.28 Basic principles of axial (b), segment (c), and angular-weighted segment (d) step depths for street network (a)
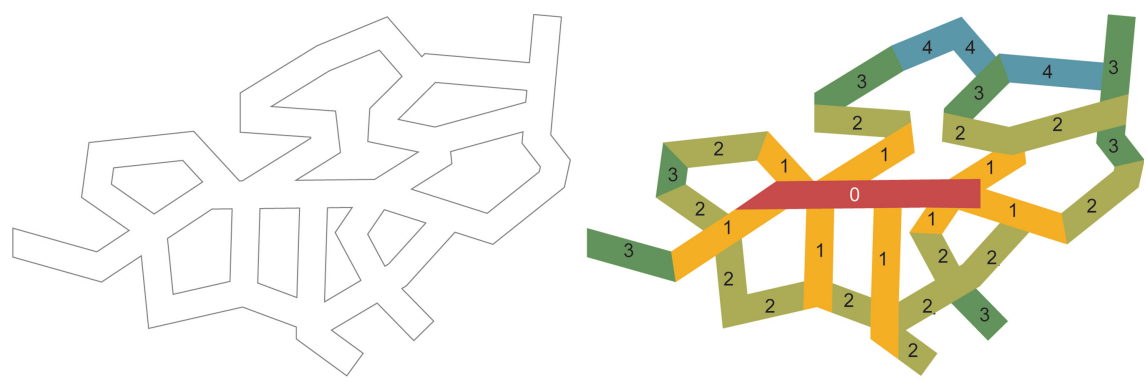

(a) The street network

(b) Axial step depth
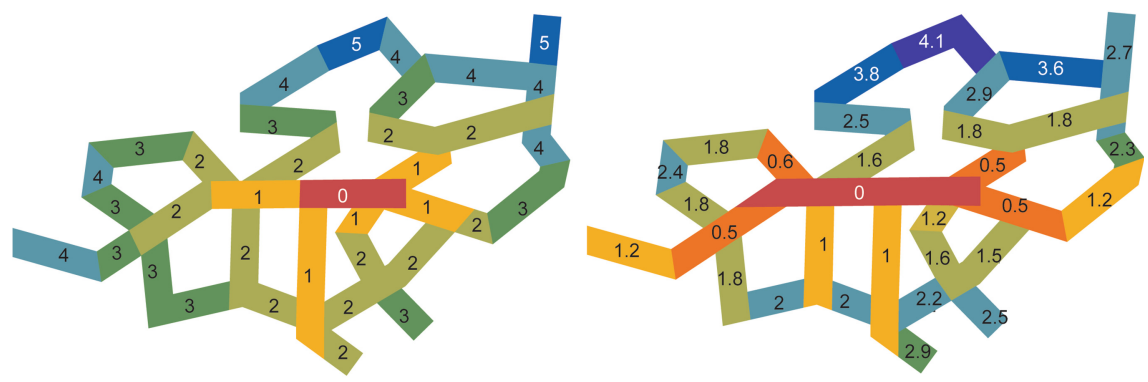

(d) Angular segment step depth

For the angular segment analysis, all changes of directions, i.e. turns, are weighted according to their angle of incidence. The first step to calculating angular choice is to calculate the mean angular depth from each segment to all others. In Fig. 2.29, we show how angular mean depth is calculated. Our example depicts four street segments connected to each other at different angles. The angular segment depth value from street segment A to B is 0.5 because the angle of incidence is $45^{\circ}$. The angular segment depth sum from A to $\mathrm{C}$ is 0.833 and is calculated from the sum of the depth of the segments $\mathrm{A}$ to $\mathrm{B}$ with an angle of $45^{\circ}$ and $\mathrm{B}$ to $\mathrm{C}$ with a total angle of $30^{\circ}\left(75^{\circ}\right.$ minus $\left.45^{\circ}\right)$. The angular segment depth from street segment $\mathrm{A}$ to $\mathrm{D}$ is also 0.833 because the angle of incidence is $75^{\circ}$. The numerical depth values are shown in the justified graph in Fig. 2.29 (right) along with the calculations of the angular mean depth or choice for street segment (A).

Fig. 2.29 Angular relationship of four street segments (right) and the justified graph with the angular weighting to all the other lines from segment A (left) (redrawn from Turner 2005, p. 148)

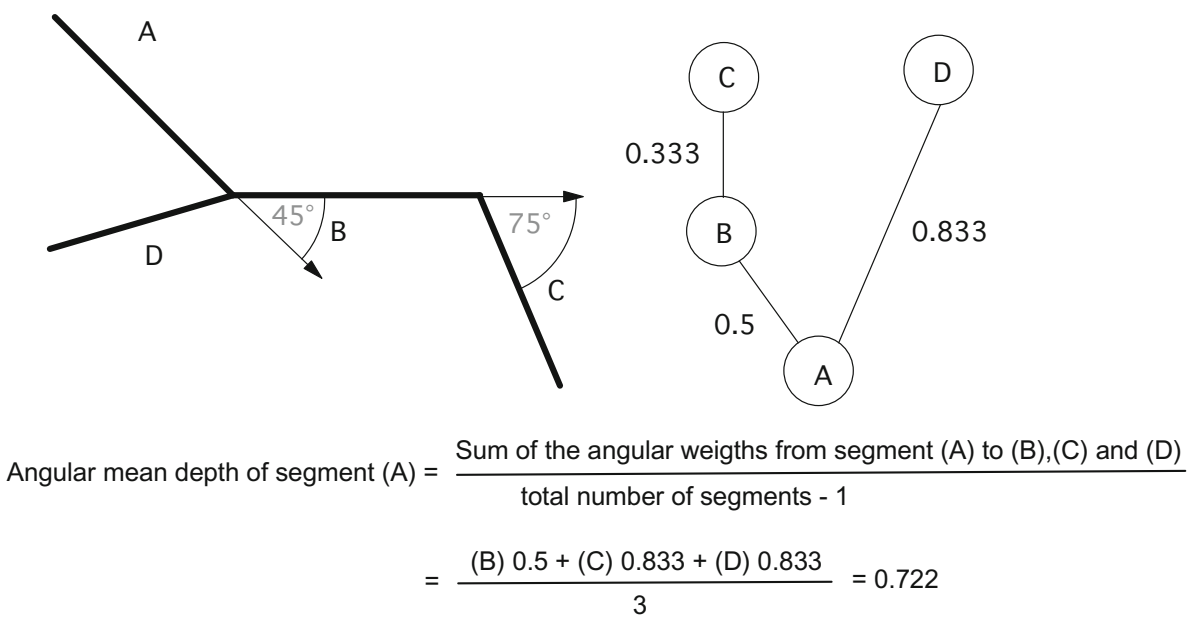

The use of the segment as a unit and the calculation of the angular mean depth of the segments was the first step to further developing the advanced calculations of the angular choice and the angular integration analyses. Before we go further, some clarification is needed for various types of distances and various types of radii. 
With regard to distances, space syntax is mostly dealing with topological and geometric distances. Metric distance is seldom used. Topological distance is about direction changes. In global axial integration analyses, only topological distances are used. Here, the globally highest integrated street has the fewest total number of direction changes to all other streets, and the most segregated street has the highest number of direction changes to all other streets. Likewise, segment integration analysis measures how integrated a street segment is in relationship to all others in terms of the fewest number of turns. The measure of integration depicts 'to-movement' potentials.

Geometric distance also takes into account the angular deviation of the direction change. A geometric distance refers to Euclidean geometry and the Manhattan distance (Fig. 2.26) with a least angular path. Thus, a geometric distance can be represented in degrees. Breaking up the axial map into segments is essential for being able to calculate geometric distances. The angular mean depth and the angular choice analysis contribute to identifying the main route network of cities and regions because these main routes have the fewest total numbers of angular deviations to all other streets in the system. Angular choice analysis highlights a route hierarchy and a route choice because these routes link how people orientate and navigate through the urban system.

Metric distance involves calculating the shortest distance from everywhere to everywhere else. Metric distance analysis measures how metrically integrated a street or road is in relationship to all others.

The application of these three types of distance in space syntax has been correlated with pedestrian flow data and location patterns of shops. Crowded shopping streets tend to be located on the most angular integrated street segments of the main route network (Turner 2005, p. 149f). The angular segment analysis (least angle) seems to be the best predictor of movement, followed closely by integration analysis (fewest turns). Metric distance is a distant third (Hillier 2005; Hillier and Iida 2005; Hillier et al. 2007).

With regard to a radius, in space syntax we are dealing with topological, geometric, and metric radii. The topological radius is about taking the number of direction changes into account. In the global axial integration analyses, we use radius $n$, and in the local axial integration analyses we use radius 2 for American cities, 5 for Persian and Arab cities, and 3 for the remaining cities. All these numbers represent the number of direction changes from each line when processing the local integration analysis.

The geometric radius is about taking the angular deviation into account in the local and global segment integration analyses. Here, we calculate how integrated each street segment is when changing a direction like $90^{\circ}$, $180^{\circ}$, etc. A main route tends to have low values for each angular step depth, and thus a main route segment tends to have a much larger extension than a local street segment. A serpentine road or street tends to have a short extension on a geometric radius due to a very sharp angular deviation for each direction change.

The metric radius is about taking metric distances into account. Here, we calculate how integrated a line is within a certain metric distance, for example, 400 and $5,000 \mathrm{~m}$. Adding the metric radius to the various angular segment integration and choice analyses shows a higher degree of correlation with socio-economic data than only the topological radius (Hillier et al. 2012). Since 2012, the metric radius has been the most used in the various angular choice and angular integration analyses.

Since the introduction of angular weighting for analysing spatial interrelationships on the segment level, various calculation methods have been developed. In articles and papers published since 2001, there exist several concepts regarding angular analyses. The most used concepts are angular choice, angular segment, local angular integration, global angular integration, angular total depth, segment integration, and angular mean depth. Two measurements have been establishedangular integration and angular choice - both with various metric radii. The angular choice is used to calculate the angular mean depth of every street segment to all others with a high and low metric radius, whereas the angular integration adds the segment length and replaces mean depth with angular mean depth in the integration formula (see Sect. 2.5.4).

\subsubsection{Radii Used in Segment Analysis}

One of the criticisms of space syntax was that it does not take metric properties into account in the analysis of the mobility networks (Ratti 2004, p. 501). Indeed, space syntax originally only worked with topological distances (number of direction changes) and geometric distances (degree of angular deviation). Metric distance was added to the angular segment analysis after 2004. 
Ansii Joutsiniemi (2005) highlights that metric properties matter, especially on a local scale. For illustrating the differences, Fig. 2.30 shows the principles of topological radius, metric radius, and geometric radius and their different analytical results, including explanations. We showcase this with a street grid example combining an orthogonal street grid and a deformed street grid.

When applying the topological distance radius 3 (= 2 direction changes and 3 syntactic steps) to the chosen street segment indicated in red, all streets in the orthogonal grid can be reached within two direction changes. The deformed grid has a low catchment area for the same topological distance (Fig. 2.30b). When applying a radius with metric distance of two units for the same street segment in red (example a), both the orthogonal and the deformed street grids have nearly the same catchment areas (Fig. 2.30 ). For the application of a radius with geometric distance (Fig. 2.30d), a radius of $180^{\circ}$ is applied. When the angle is sharp (below $90^{\circ}$ ) the value is high, and when the angle is shallow (above $90^{\circ}$ ), the value is low.

In this example, for every direction change from the chosen street segment marked in the black frame, the angular deviation is added up until $180^{\circ}$ is reached. The catchment area of the red segment is larger in the upper part of the red line than the lower part of the deformed street grid. This is due to the different angular relationships between the segments between the upper and the lower parts in relation to the chosen starting segment. In the lower part, the first step from the segment has a sharp angle, which gives a high value on the first step. Main routes tend to have very shallow angles. Here in our example, the segments connected to the segment in the black frame have an angle of zero. Therefore, these segments get low values and are coloured in red.

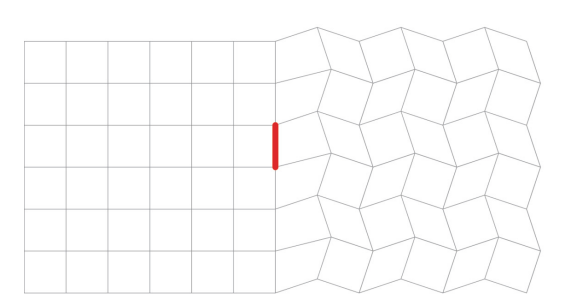

(a) Marked street segment in red (= root segment)

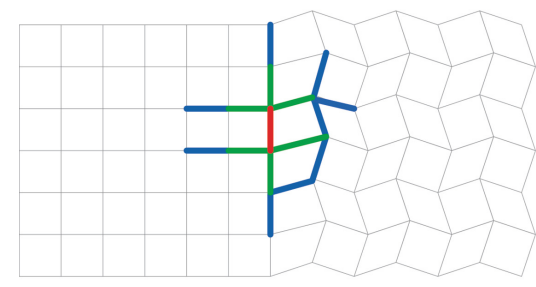

(c) Metric radius $=$ two units from the red segment

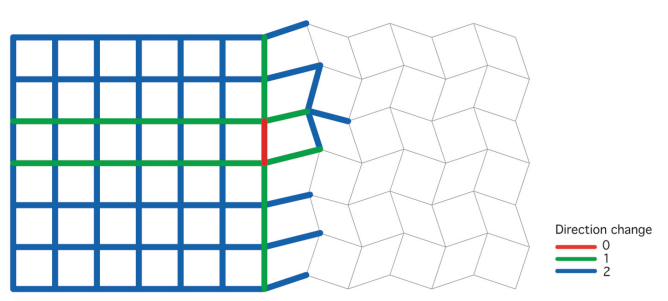

(b) Topological distance radius $=3$ (two direction changes)

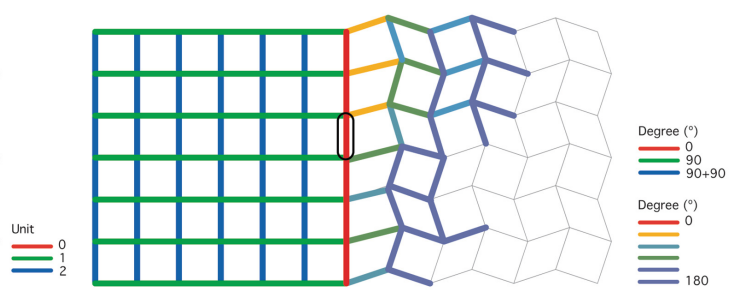

(d) Geometrical radius $=$ total 180 degrees from the red segment

Fig. 2.30 The principles of topological (b), metric (c), and geometric (d) radii

Adding a metric radius to the angular segment and angular choice analyses provides a much more fine-grained analysis than the axial analysis. In Fig. 2.31, we apply the same metric radius of $900 \mathrm{~m}$ to topological, metric, and geometric distances in Amsterdam. All distances are based on the segment map.

The topological distance (the number of direction changes) is a segment integration analysis with a metric radius of $900 \mathrm{~m}$ (image (a)). The Pijp area has the highest integrated streets, and the area is perceived as the main centre of Amsterdam for the locals. This area is known to be highly vital and a lively local centre for the entire city. Pijp offers a large variety of shop types and has crowded streets throughout the day. All locations within Pijp are easily accessible by bicycle, tram, and car and partly on foot.

The metric distance with a metric radius analysis of 900 metres (Fig. 2.31b) reveals that the metric centre does not always overlap with a city's economic centre or where the vital centre tends to be. In Amsterdam, the results from the metric distance with metric radius shows a patchwork of local neighbourhoods with a particular local identity. In particular, the neighbourhoods Geuzenveld, Appollobuurt, Van der Pekbuurt, Watergraafsmeer, Diemen, Duivendrecht, and Slotervaart are highlighted in red. These spots are not vital local centres; rather, they are neighbourhoods with a clear identity and a name. 


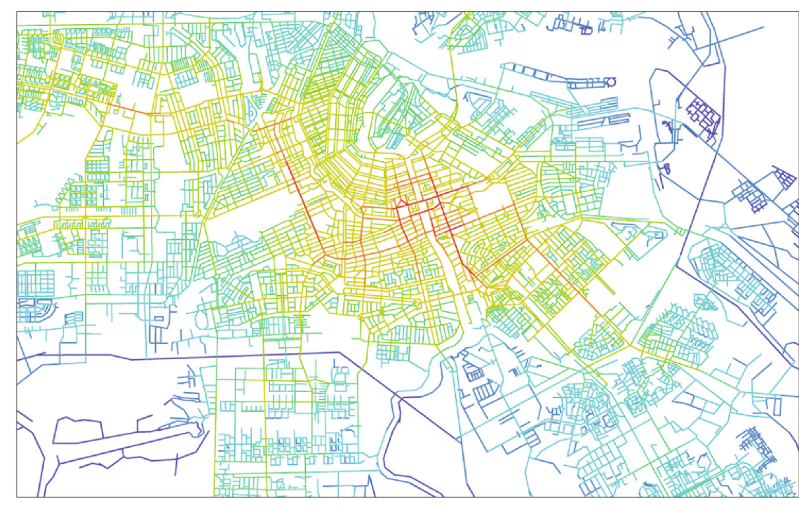

(a) Topological distance with metric radius $=900$ meters

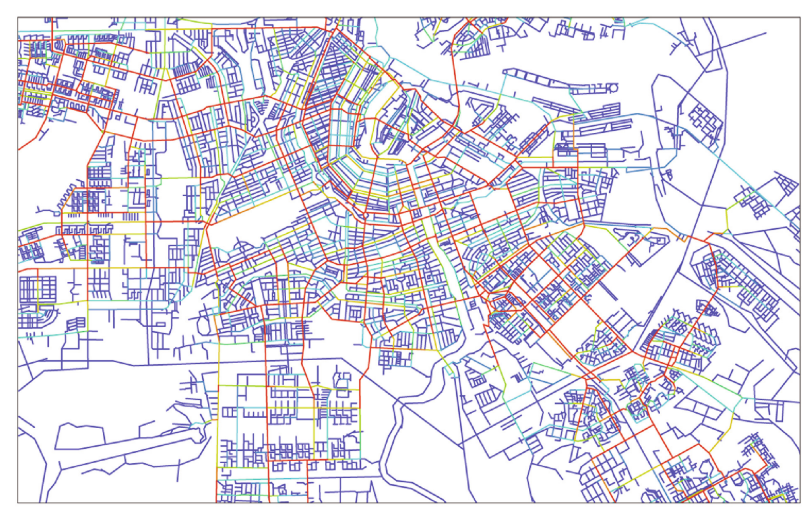

(c) Geometrical distance with radius $=900$ meters

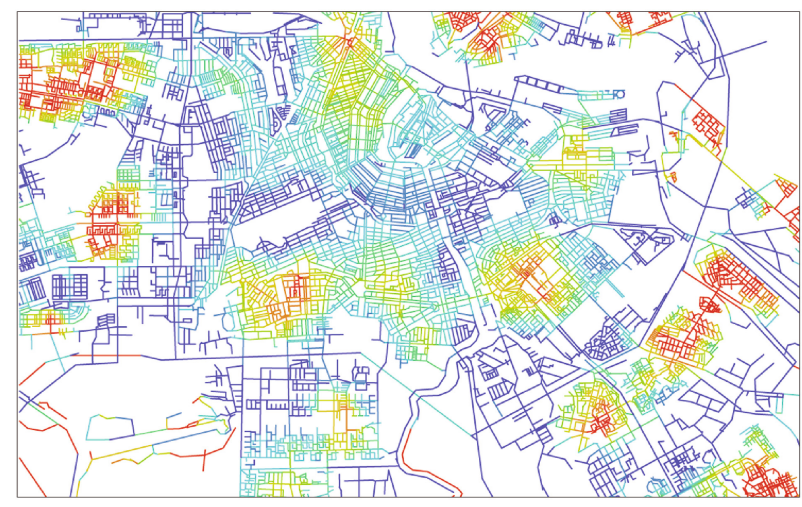

(b) Metric distance with metric radius $=900$ meters

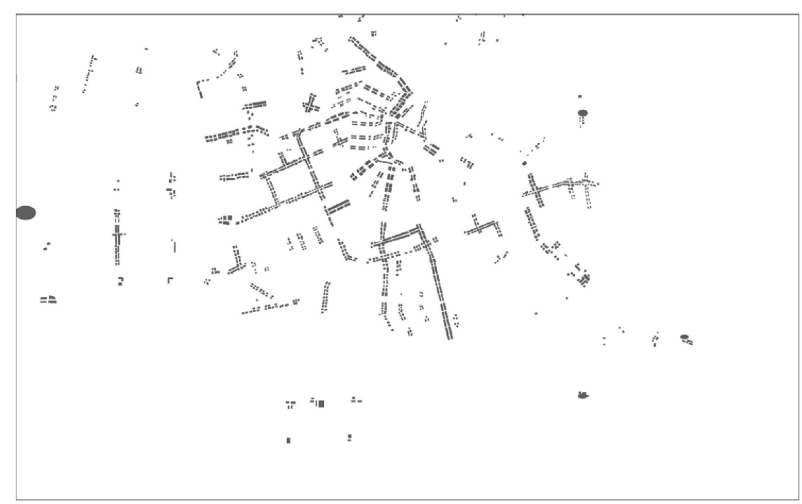

(d) Location pattern of shops

Accessibilty

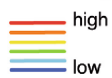

Fig. 2.31 Applying the same metric radius to different distances on Amsterdam: (a) topological distance with a metric radius of $900 \mathrm{~m}$, (b) metric distance with a metric radius of $900 \mathrm{~m}$, (c) geometric distance with a radius of $900 \mathrm{~m}$, and (d) the location pattern of shops

The geometric distance (the degree of angular deviation) with a metric radius of $900 \mathrm{~m}$ (Fig. 2.31c) highlights the main route network. These main routes have the fewest angular deviations to all other streets within a radius of $900 \mathrm{~m}$. This geometric distance analysis is the logic of the angular choice analysis. Amsterdam's location pattern of shops (Fig. 2.31d) and tramline routes follows the street segments with the highest values in the angular choice analysis with a metric radius of $900 \mathrm{~m}$ (Fig. 2.31c).

Following this, we apply the most used analyses to Amsterdam (Fig. 2.32). For the axial integration analysis (a) and angular choice analysis (b), we apply a topological radius of 3. Here, we calculate how integrated every axis or street segment is when changing directions three times from them (according to the latest developments in space syntax; see for further explanation Yamu et al. 2021). In both analyses, Amsterdam's oldest city centre around the Berlage stock exchange (inside the black circle) is poorly integrated.

In Fig. 2.32 we show the latest calculations based on segments within a metric radius. Image (c) shows an angular integration and image (d) shows an angular choice analysis, both with a radius of $400 \mathrm{~m}$. Now, the streets inside Amsterdam's oldest centre have the highest integration values in both analyses. This area is neither highlighted in the global and local axial integration analyses nor in the global angular segment analysis with various topological radii. This is because the area around the old Berlage stock exchange building was originally developed with short metric accessibility to other functions. Today, it is Amsterdam's tourist centre where everything is accessible on foot. 


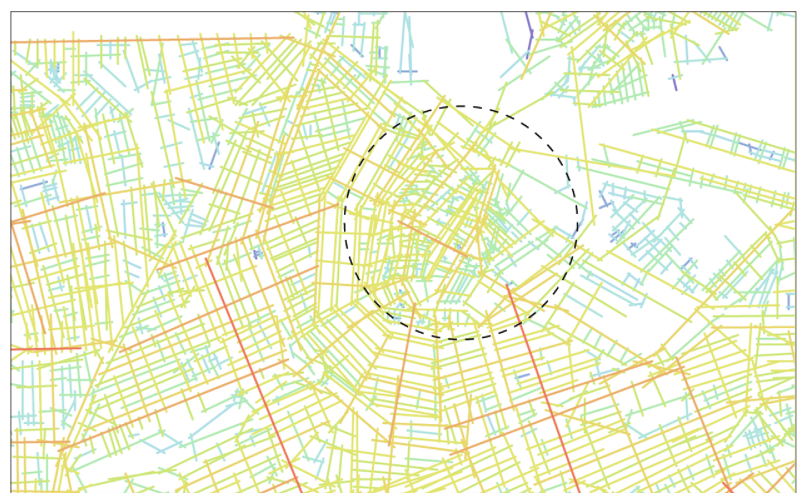

(a) Axial integration with topological radius $=3$

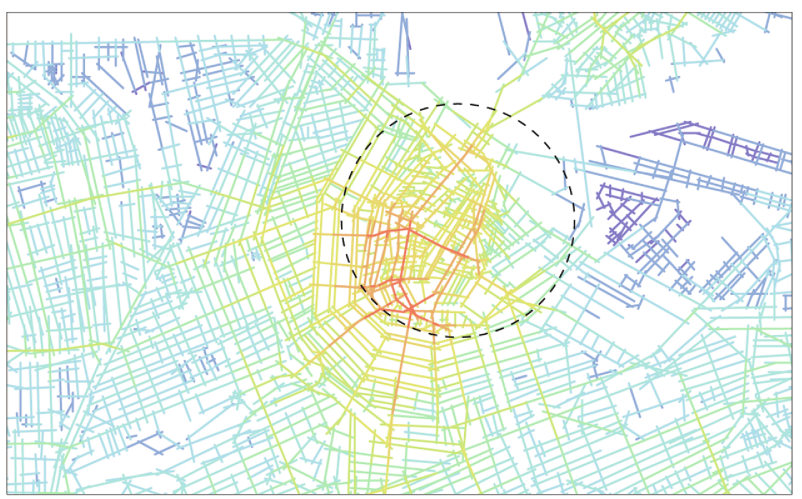

(c) Segment integration with radius $=400$ meters

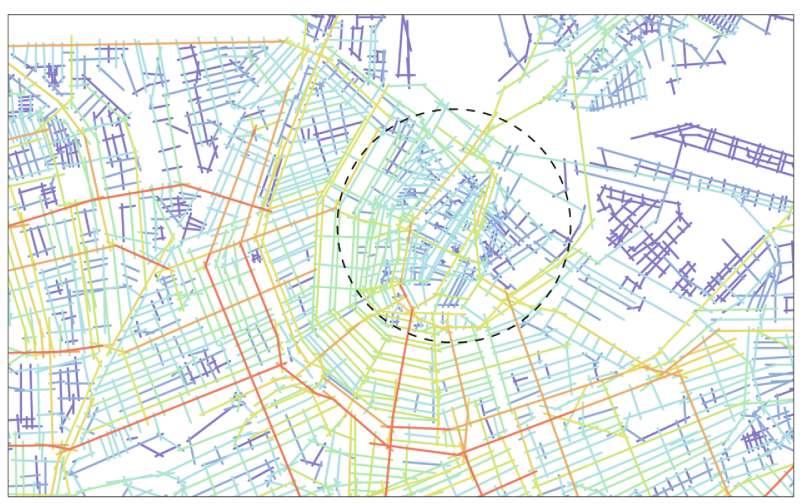

(b) Angular segment integration with topological radius $=3$

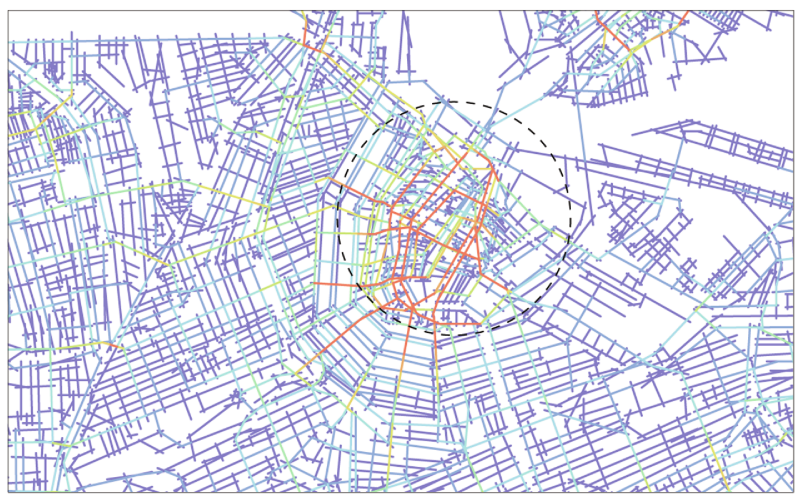

(d) Angular choice with radius $=400$ meters
Accessibilty

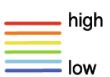

Fig. 2.32 Various local measurements of Amsterdam, with the location of the oldest centre of Amsterdam around the old Berlage stock exchange indicated in the black circle

Metric radius matters for the angular segment integration analysis as well as for the angular choice analysis. Other radii, for example, of 800 or $1200 \mathrm{~m}$ highlight various local urban centres. An indication of the size of the local catchment areas is often given by the various values of the metric radii. Most commonly, a radius of 4,000-8,000 $\mathrm{m}$ is used as a high metric radius and a radius of $400-800 \mathrm{~m}$ is used for a low metric radius. For axial maps that are scale free or non-georeferenced, the local or low radius tends to be $10 \%$ of the global or radius $\mathrm{n}$, also called the high radius.

Adding metric radii (e.g. $400 \mathrm{~m}$ or $800 \mathrm{~m}$ ) to the angular choice analysis allows a more sophisticated spatial analysis of urban networks compared to axial analysis with its topological radii (e.g. radius 3 or radius 5). To show what metric radii add to space syntax on a local scale, we show in Fig. 2.33 five different spatial analyses of Friedrichstraße in Berlin including a non-processed axial map with the distribution of shops (f). Images (a), (b), and (c) show the results from the segment analyses. Image (a) is the angular choice analysis with a topological radius of 3. Here, Friedrichstraße is a part of the main route system. Image (b) shows an angular choice analysis with a metric radius n. Here the northern part of Friedrichstraße is highlighted in red. At present, Berlin's largest shopping malls are located at this spot. Image (c) shows an angular choice analysis with a low metric radius. Now the central and southern parts of Friedrichstraße also get higher values. The local shops serving the neighbourhood are located there. The segment length is included in the analyses in images (b) and (c), whereas it is not taken into account in image (a).

The segment analyses with segment length and with metric radii show more sophisticated results than the axial global (Fig. 2.33d) and local (Fig. 2.33e) integration analyses. In the axial analyses, Friedrichstraße gets the same value for its whole length, whereas in the segment analyses various parts of the street are highlighted depending on the radius. Seemingly, the size of the metric radius indicates where the customers for the shops come from, whether it is only from the immediate vicinity or the whole city. 


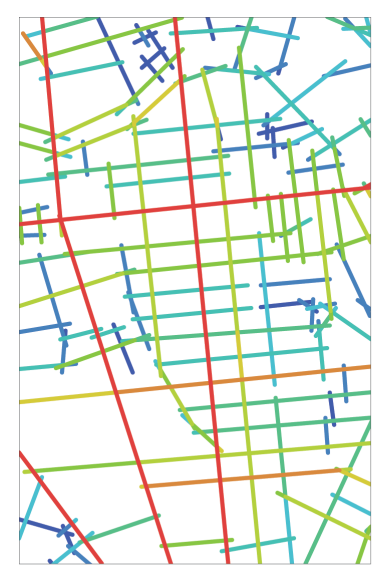

(a) Angular choice with topological radius 3

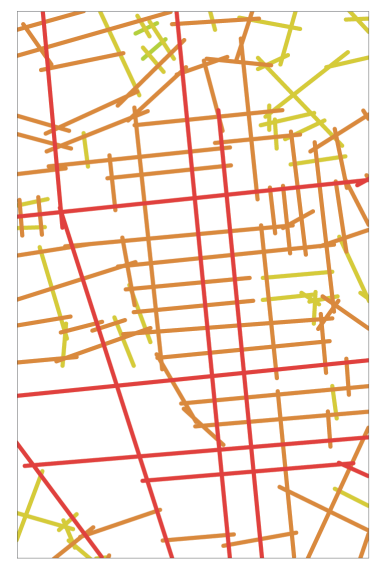

(d) Global axial integration with radius $n$

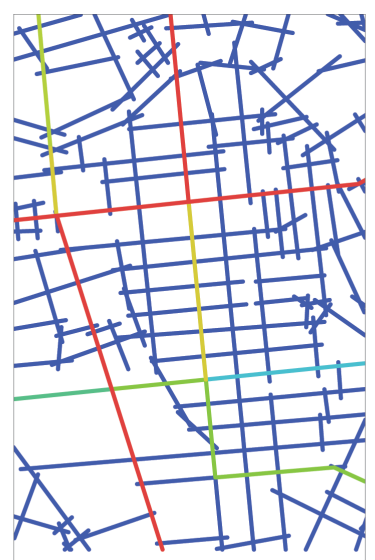

(b) Angular choice with metrical radius $\mathrm{n}$

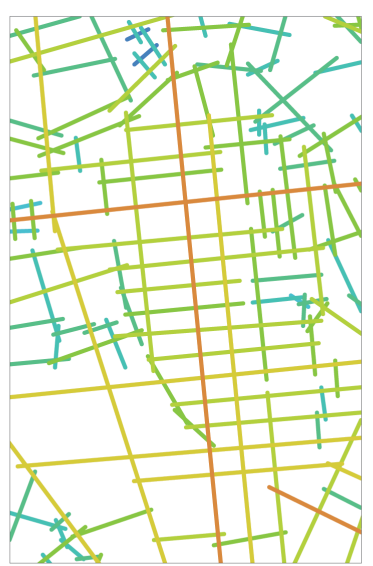

(e) Local axial integration with radius 3

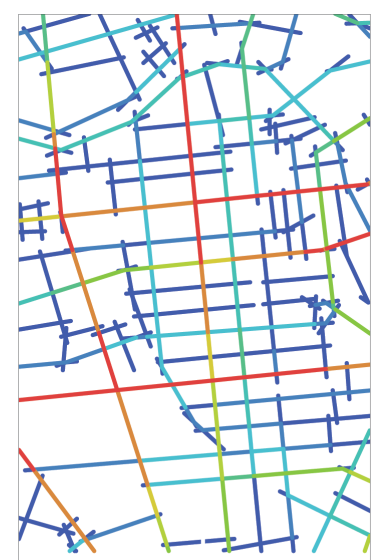

(c) Angular choice with metrical radius $500 \mathrm{~m}$

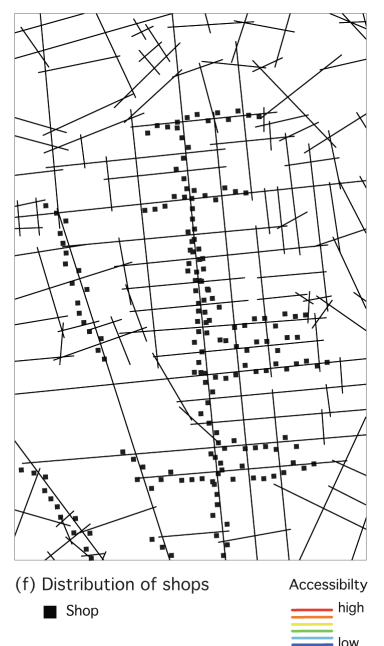

Fig. 2.33 Angular segment $(\mathbf{a}, \mathbf{b}, \mathbf{c})$ analysis and axial analysis $(\mathbf{d}, \mathbf{e})$ with the location pattern of shops (f) for the Friedrichstraße area in Berlin

In experiments testing various types of distances with various types of radii and correlating the results with socio-economic data, two types of spatial analyses are most commonly used - angular choice and angular integration with a low and a high metric radius. These measurements will be discussed in the next sections.

The most used metric radii when having a georeferenced axial map or road centre line map are 400-800 and 5,000-8,000 m. The first set of radii concerns walkable distances or local accessibility, whereas $800 \mathrm{~m}$ refers to a 10 minute walk. The latter set of radii concerns car-based distances or citywide accessibility. In general, we apply the $10 \%$ rule, which means that the low radius is $10 \%$ of the value of the high radius. When the segment map is not georeferenced, we apply a $10 \%$ rule retrieved from the system's coordinates for a local radius. Radius $\mathrm{n}$ stays the same. The system's coordinates are often determined by the space syntax software.

\subsubsection{Angular Choice: Main Routes Through Cities and Regions}

The angular choice analysis depicts the 'through-movement' potential and therefore an urban route hierarchy. When applying various metric radii for the angular choice analysis, the following results are obtained. For a small metric radius, the local main routes of local centres of a city are highlighted. This applies, for example, for radii of 400, 800, or 1,200 m. The higher the applied metric radius, the more the main routes running through and between neighbourhoods in a city are highlighted. In the radius $\mathrm{n}$ analyses, the highway network tends to get highlighted.

According to Hillier et al. (2007), cities have a dual nature with a foreground (citywide or 'global') and a background (local) street network. The foreground network links urban centres at all scales and levels. It gives a city's street structure a 
so-called 'deformed wheel pattern' consisting of radials and orbitals. This structure can even be seen in cities having an orthogonal street grid. The main route network through urban areas shapes the deformed wheel's armature. In this way, accessibility from the city's edges to its centre and the natural interface of co-presence through movement from centres to edges is made efficient and possible. The foreground street network works independently of cultures. Correspondingly, cities have a background network for residential areas, which reproduces cultural patterns. Different cultures have different local radius measures, which are the conservative components of the city. The background local network is mostly shaped by the way different cultures influence urban space, including both the metric and geometric properties of the network. However, local residential areas tend to also have a localised foreground structure (Hillier et al. 2007, p. 4).

However, we can observe that all cities and towns are made up of a very large number of short streets and a very small number of long streets and roads (Figueuredo and Amorim 2005, p. 163). This can be identified across scales and gives a city's street network and a regional road network a clearly fractal structure (Czerkauer-Yamu 2012; Yamu and van Nes 2017, 2019). In addition, Claudia Yamu says that the fractal structure of the urban street network and the metropolitan road network incorporates an intrinsic hierarchy and both are therefore rather consistent with fractal ordering principles (Yamu and Frankhauser 2015; Yamu et al. 2016; Yamu 2014). Hillier explains that the foreground network is largely composed of longer streets and roads that connect to other streets and roads at highly obtuse angles. The longer the street line, the more likely it is to end with a nearly straight connection. Main routes through cities on all scales tend to consist of a set of longer lines connected to each other with almost $180^{\circ}$ angles (Hillier and Iida 2005). Herein, angular weighting contributes to highlighting the main routes in the angular choice analysis (van Nes and Yamu 2018). Conversely, the background network is largely made up of short streets that tend to intersect with other streets at nearly right angles. The shorter the street, the more likely it is to end at a right angle. Most quiet residential streets tend to be metrically short (Hillier et al. 2007 pp. $2 \mathrm{ff}$ ).

According to cognitive science, human beings seem to choose the straightest routes to avoid route complexity (Dalton 2001). Figure 2.34 shows two street examples from the city of Cologne, Germany, including a short residential street connecting with an angle of $90^{\circ}$ to its adjacent street (left) and a long main route connecting to the next street at an angle of almost $180^{\circ}$. Both examples are taken from the same neighbourhood.
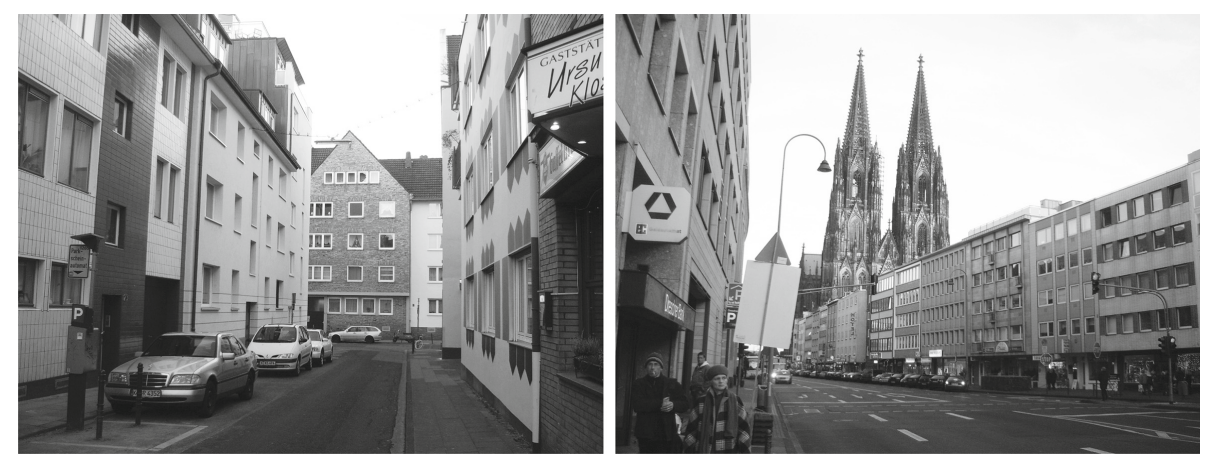

Fig. 2.34 It shows a short background network street (left) and a long foreground network street (right) in the city of Cologne, Germany

In Fig. 2.35, we illustrate the foreground and the background network for the city of Amman in Jordan (left) and Banda Aceh in Indonesia (right) through an angular step depth analyses taken from the main route network. The main routes are identified by applying an angular choice analysis with radius $\mathrm{n}$. These main routes are coloured in red. From the main route network, a radius of $\mathrm{n}$ degrees is used in the angular depth analyses. Applying both analyses, we can see how the background network is connected to the main route network in terms of the total number of angular deviations.

For Amman, the analysis depicts a typical Middle Eastern city with a well-integrated foreground network and a segregated background network. The background network is represented in dark blue. The various local neighbourhoods (marked in blue and turquoise) are not visible from the main route network. This complies with the local culture and religion in that the domestic spaces and the streets these spaces are connected to are more protected from random through travellers than in cities in Europe, North America, East Asia, or Central and South Africa. 

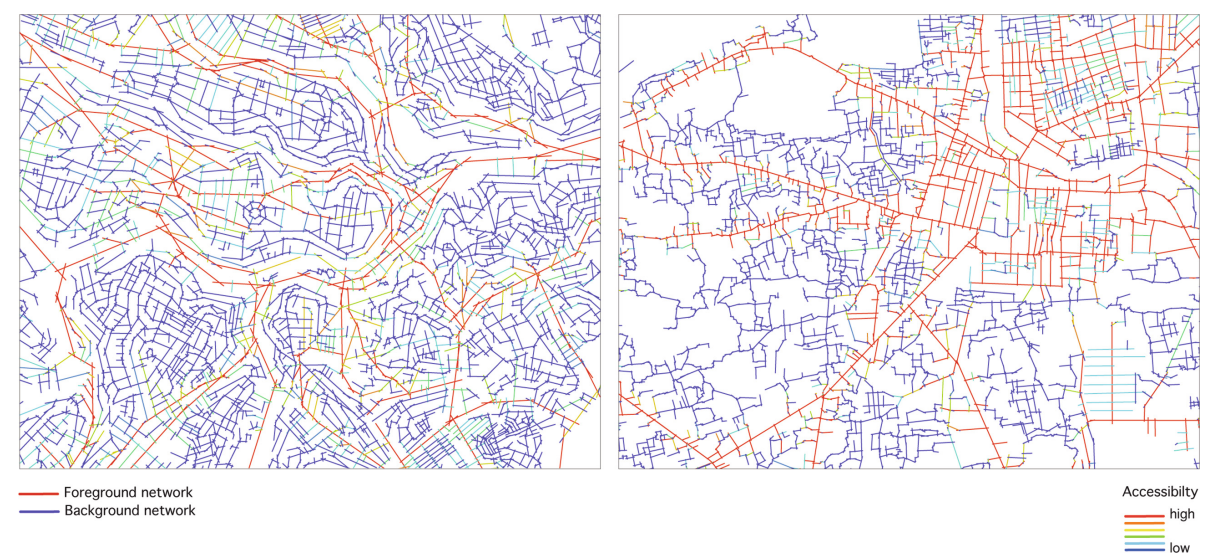

Fig. 2.35 Foreground and background networks for Amman in Jordan (left) and Banda Aceh in Indonesia (right)

In contrast, Banda Aceh is an Islamic city located in Indonesia. Its street pattern was mostly developed during the Portuguese and Dutch colonial rule from 1600 to 1940. For Banda Aceh, a majority of the background network is easily accessible from the foreground network. The foreground network has a high density. The city incorporates both types of structures: a tree structure and a network structure. The tree structure in the west of the city is poorly accessible from the main route network. This part of the city was developed in the time period dominated by the Islamic culture. The city's network structure in the east was developed under colonial rule.

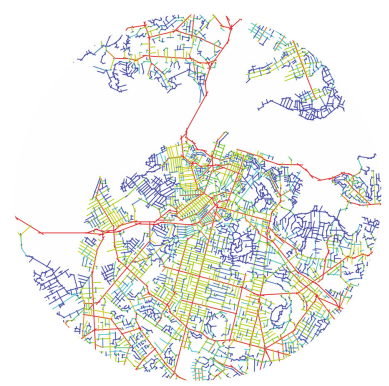

Australia \& New Zealand: Auckland

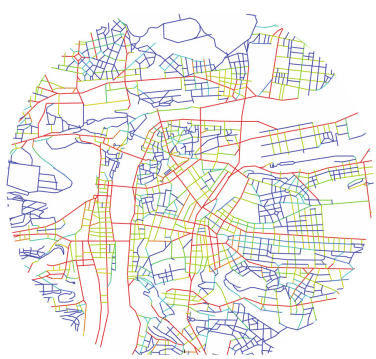

Europe: Prague

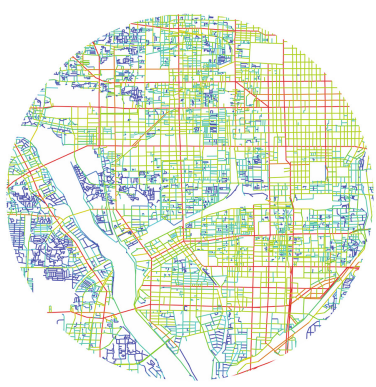

Asia: Kyoto

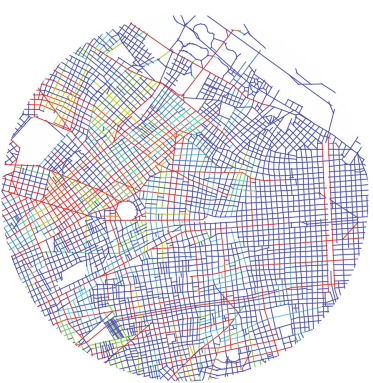

South America: Buenos Aires

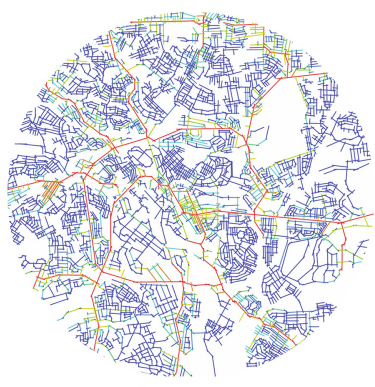

Africa: Kumasi

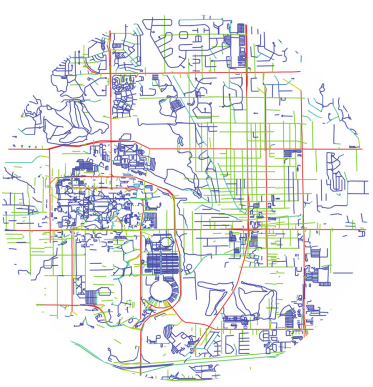

North America: Ames, lowa

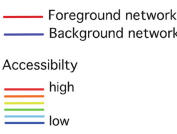

$\overline{\bar{\equiv}}_{\text {low }}$

Fig. 2.36 Foreground and background networks for six cities from six different continents 
Moving on, we present six further city examples from six different continents with different urban typologies on how foreground and background networks establish themselves (Fig. 2.36). Again, we applied angular step depth analysis from the main routes. The established foreground and background networks vary from city to city and culture to culture. Likewise, the colour scale of the background network varies for each city. For some cities, the background network is better connected to the foreground network than for others.

The cities of Kyoto, Ames in Iowa, and Buenos Aires have an orthogonal grid structure. However, the way the background network is connected to the foreground network is different. Kyoto has a far more accessible background network than Buenos Aires and Iowa. The cities of Prague, Kumasi, and Auckland have a main route network forming a 'deformed wheel' pattern. In Kumasi, the new bypass road (or ring road) has become a part of the foreground network. In Auckland centre and Prague, the background network is well connected to the foreground network. A feature in most European cities is that in the pre-war urban areas, the background network is well connected to the foreground network. In the post-war or modernist urban areas, the background network is poorly connected.

Following up the discussion about the foreground and background networks, we demonstrate in the following what different citywide and local radii mean for urban centrality. We apply the angular choice analysis to the two Dutch towns of Lelystad and Hilversum.

The new town of Lelystad was founded in the late 1960s and is located on reclaimed land from the Zuiderzee (english: Southern Sea). Car lanes, bicycle lanes, and pedestrian sidewalks are separated from each other. Figure 2.37 shows an angular choice analysis applied to an axial segmented map for the street network with (a) radius $6000 \mathrm{~m}$ and (b) radius $600 \mathrm{~m}$.

For the angular choice analysis with a radius of $6,000 \mathrm{~m}$, all main routes between various local centres are highlighted in red to green. These routes have predominantly vehicular traffic. Lelystad's main shopping mall is located in the geometric centre of the town where the density of these main routes is the highest. In contrast, the angular choice analysis with a radius of $600 \mathrm{~m}$ depicts the local centres of Lelystad. Streets indicated in red are locations where small neighbourhood supermarkets and corner shops are located. However, the vitality of these local centres is very low. In addition, Joris van Casteren (2008) states that a criticism directed at new towns is that they tend to be 'dormitory towns'. Analysing new towns using angular choice with metric radii can shed some light on this discussion.

When applying the same analysis to the historic town of Hilversum close to Amsterdam, a different spatial structure can be identified (Fig. 2.38). Hilversum is one of the oldest settlements in the Netherlands, founded in 1424. Angular choice analysis with a radius of $6,000 \mathrm{~m}$ depicts a deformed wheel structure constituted by the main road system. In contrast, the angular choice radius of $600 \mathrm{~m}$ highlights the town's local shopping streets, indicated in red and orange. Both analyses depict all of Hilversum's central shopping streets. As mentioned earlier, optimal locations for shops are streets that are accessible within a short metric distance to the neighbourhood's residents and close to streets with citywide through- and to-movement potential. The variations of shops in local shopping areas tend to be higher in historic towns compared to new towns. Often, newer Dutch towns have a small neighbourhood supermarket, mostly used by the neighbourhood's residents. In contrast, historic Dutch towns have a good variation of shops in strategic locations in streets that can be easily reached from the main route network.

Overall, the main route network in newly built neighbourhoods is separated from the local centres, whereas it is integrated in historic towns. Results from various space syntax analyses indicate that spatial conditions for generating vital urban areas with a high diversity of active land use are as follows: (1) there is a topologically and geometrically well-integrated street network from a local to a global scale; (2) main routes have high accessibility values for angular choice global radius analysis; (3) main routes are running through neighbourhoods instead of running around them; (4) main routes are directly connected to a majority of local residential streets; and (5) the same main routes are running through or are well connected to a locally integrated centre of a town or city. 


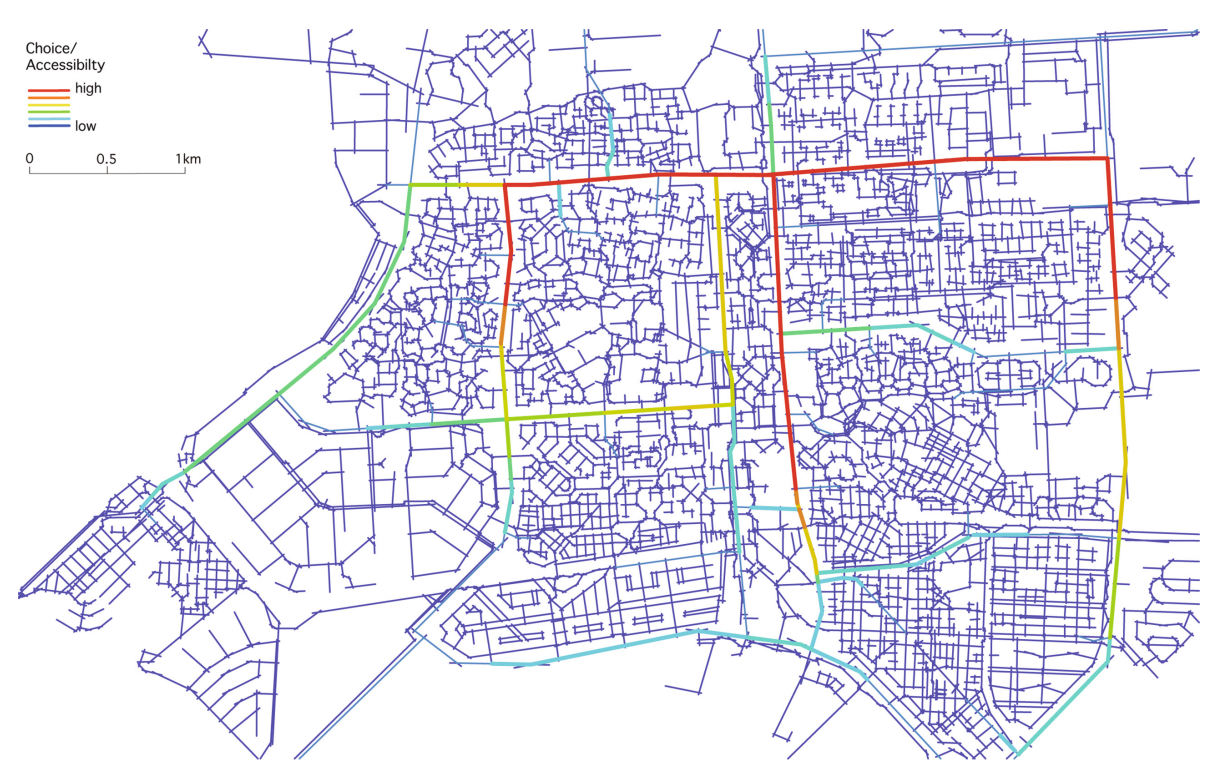

(a) Lelystad: Angular choice, radius $6000 \mathrm{~m}$

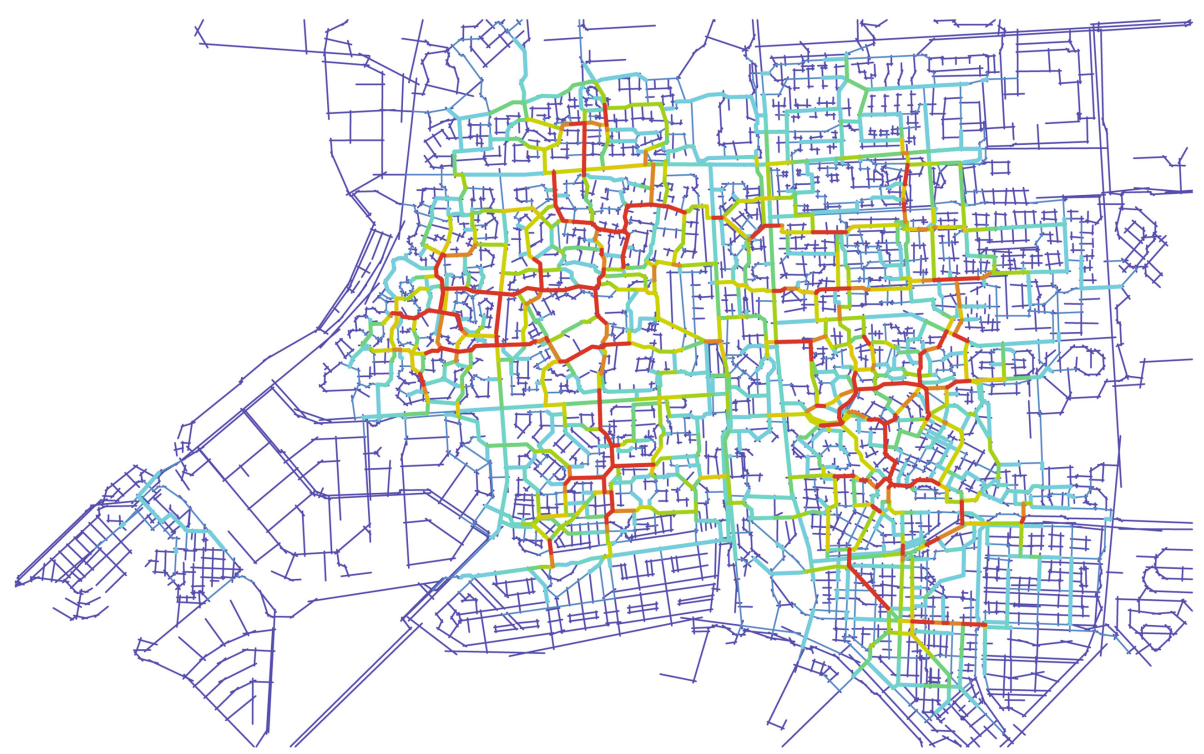

(b) Lelystad: Angular choice, radius $600 \mathrm{~m}$

Fig. 2.37 Angular choice analysis for the town of Lelystad with (a) a radius of $6,000 \mathrm{~m}$ and (b) a radius of $600 \mathrm{~m}$

\subsubsection{Angular (Segment) Integration: The Location of Urban Centres}

The angular segment integration shows the accessibility of a street segment in relation to all other street segments in an urban system in terms of direction changes. For this kind of analysis, the street segment's connectivity represents the 'to-movement' potentials for the city or town under scrutiny. As mentioned earlier, connectivity is the basis for integration and is the underlying logic for segment integration analysis. Segment connectivity consists of counting the number of connections from a street segment to all other adjacent street segments. The unit of segment connectivity is the individual street segment, which is a street section from junction to junction (Hillier and Sahbaz 2005, p. 455f). Segment connectivity is the basis for calculating angular integration by means of mean angular depth. 
Fig. 2.38 Angular choice analysis for the town of Hilversum with (a) a radius of $6,000 \mathrm{~m}$ and (b) a radius of $600 \mathrm{~m}$

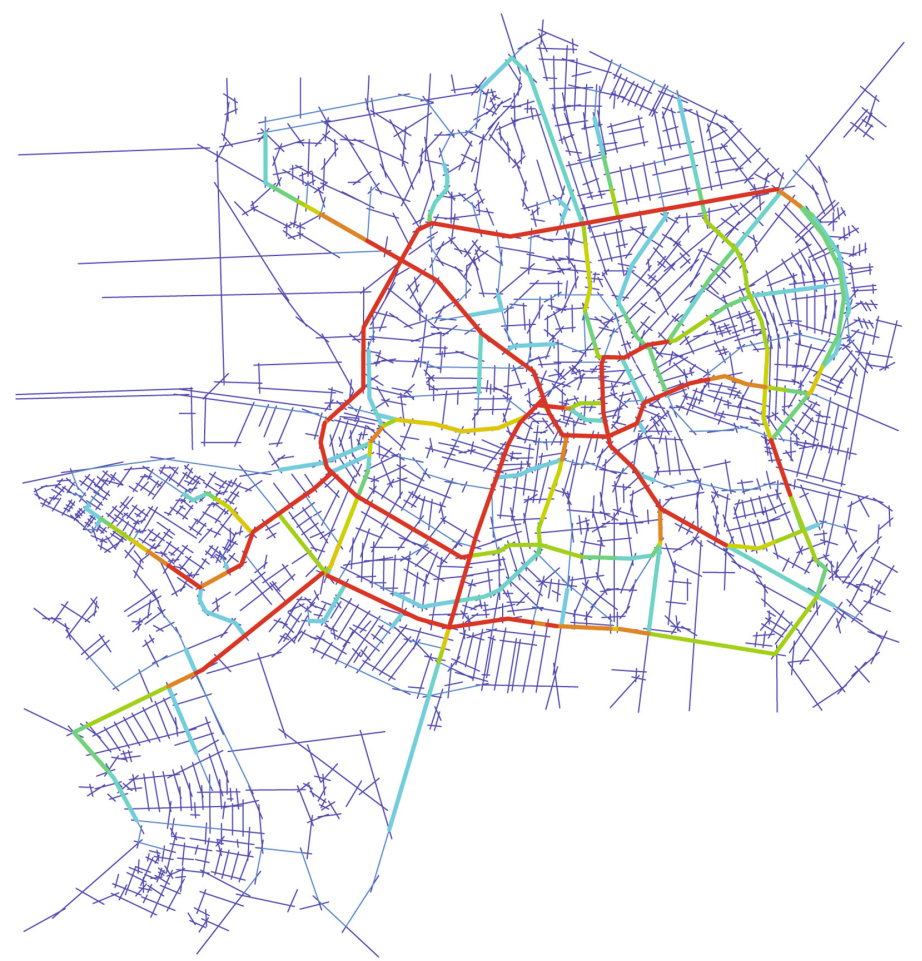

(a) Hilversum: Angular choice, radius 6000m

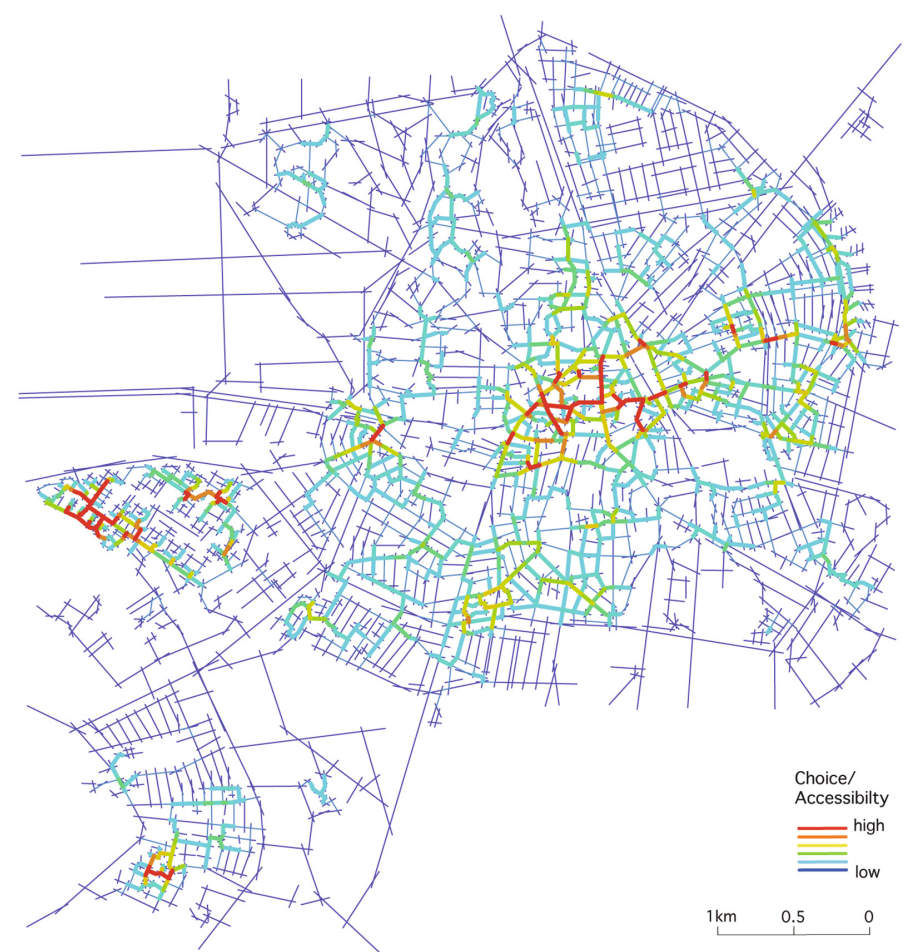

(b) Hilversum: Angular choice, radius $600 \mathrm{~m}$ 
Figure 2.39 illustrates how segment connectivity is calculated. For our example (a), the chosen street segment on a throughway has six connections, thus its connectivity is six. The street segment from example (b) has a connectivity of three with one direction change from the throughway and a connectivity of one with two direction changes away from the throughway. For example (b), it is possible to hide away in the cul-de-sac or to escape via the adjacent T-junction.

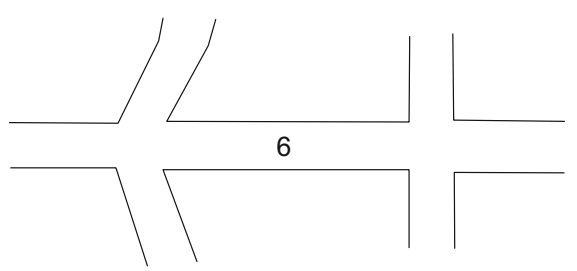

a)

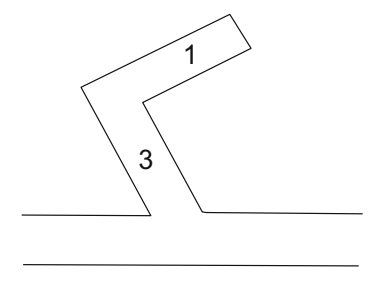

b)

Fig. 2.39 Segment connectivity for two different sets of streets: (a) represents a through carry way in traditional urban areas with two X-junctions and (b) represents a cul-de-sac with an adjacent T-junction

The usefulness of segment connectivity analysis is to highlight potential escape routes for criminal activities or exit routes when fleeing from natural hazards like tsunamis. Connectivity is a local static measure. In general, main streets tend to have high segment connectivity, while dead-end streets tend to have low segment connectivity (Hillier and Sahbaz 2005, p. 456). In studies on urban safety and crime, all possible escape routes, for example, back paths and footpaths through parks are taken into account in the segment connectivity analysis (Hillier and Sahbaz 2005; López and van Nes 2007).

Angular segment integration analysis seeks to measure the to-movement potentials on a neighbourhood and a citywide scale. The same mathematical logic is applied to angular segment integration analysis as in the axial integration analysis. The only difference is that it is the segment that is the unit for analyses, segment length is taken into account, the topological mean depth calculation from the axial analyses is replaced with the angular mean depth or angular choice analysis, and that metric radii can be added. In order to show the principle difference between segment integration and angular choice analyses, Fig. 2.40 shows two different types of j-graphs on how to calculate the choice (C) and the segment mean depth (MD) relationship from segment A.
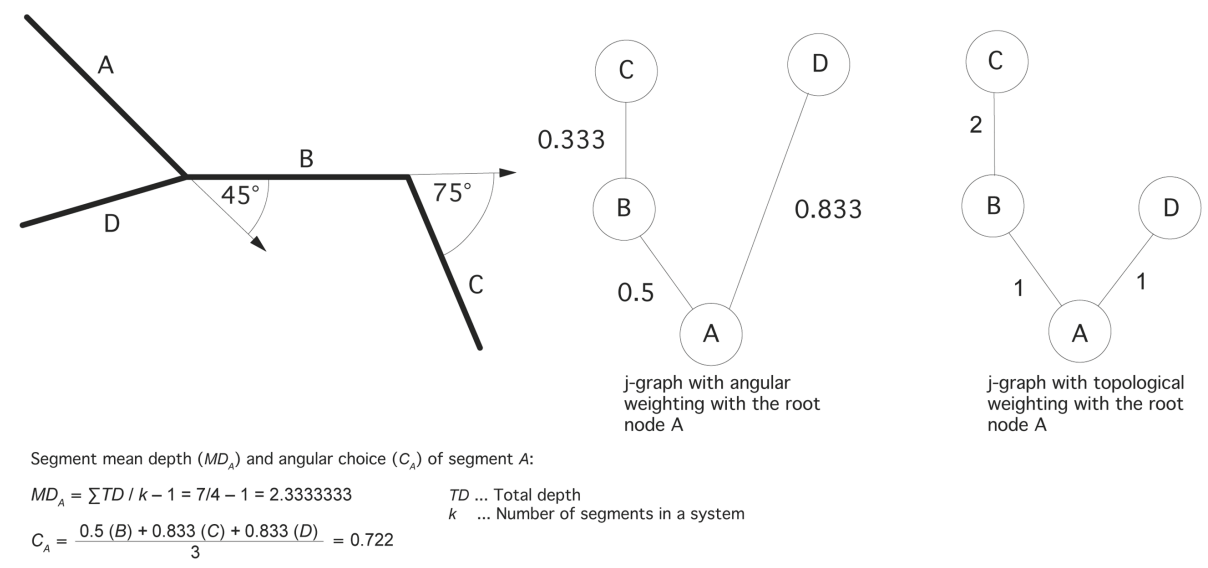

Fig. 2.40 Two different types of j-graphs for calculating the mean depth from segment A to segments B, C, and D 


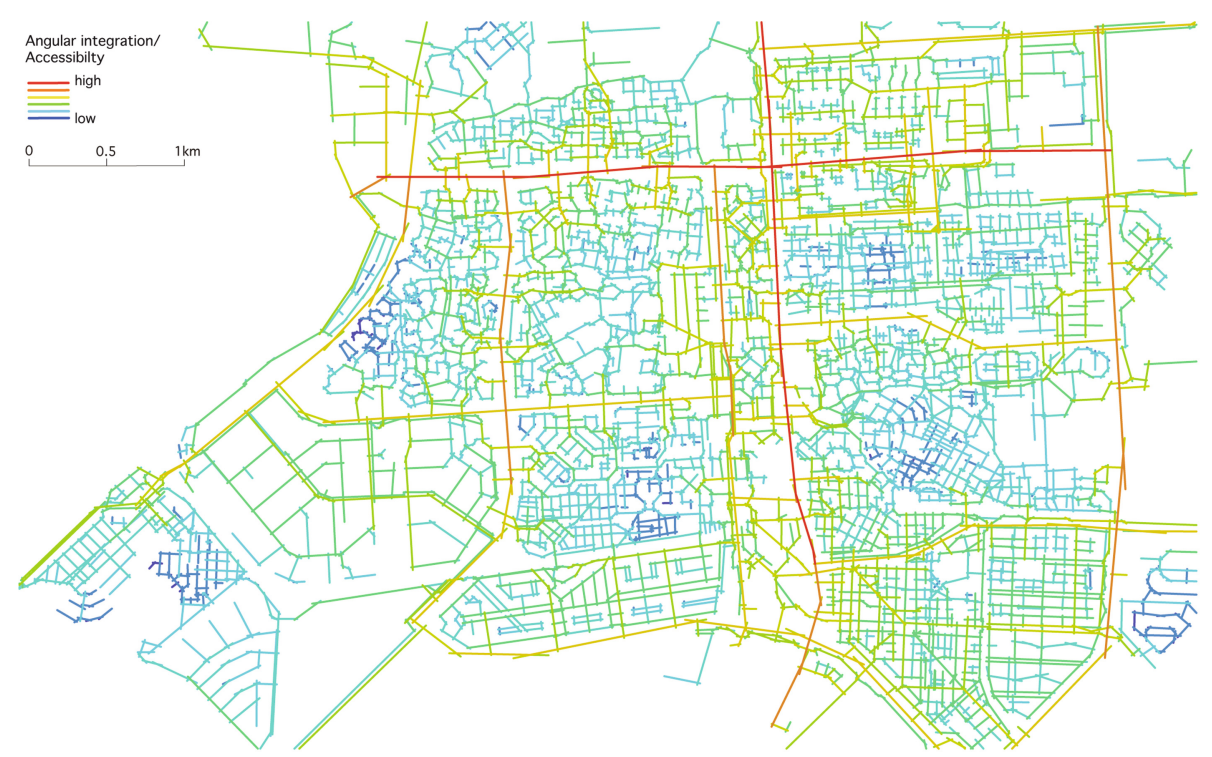

(a) Lelystad: Angular (segment) integration, radius $6000 \mathrm{~m}$

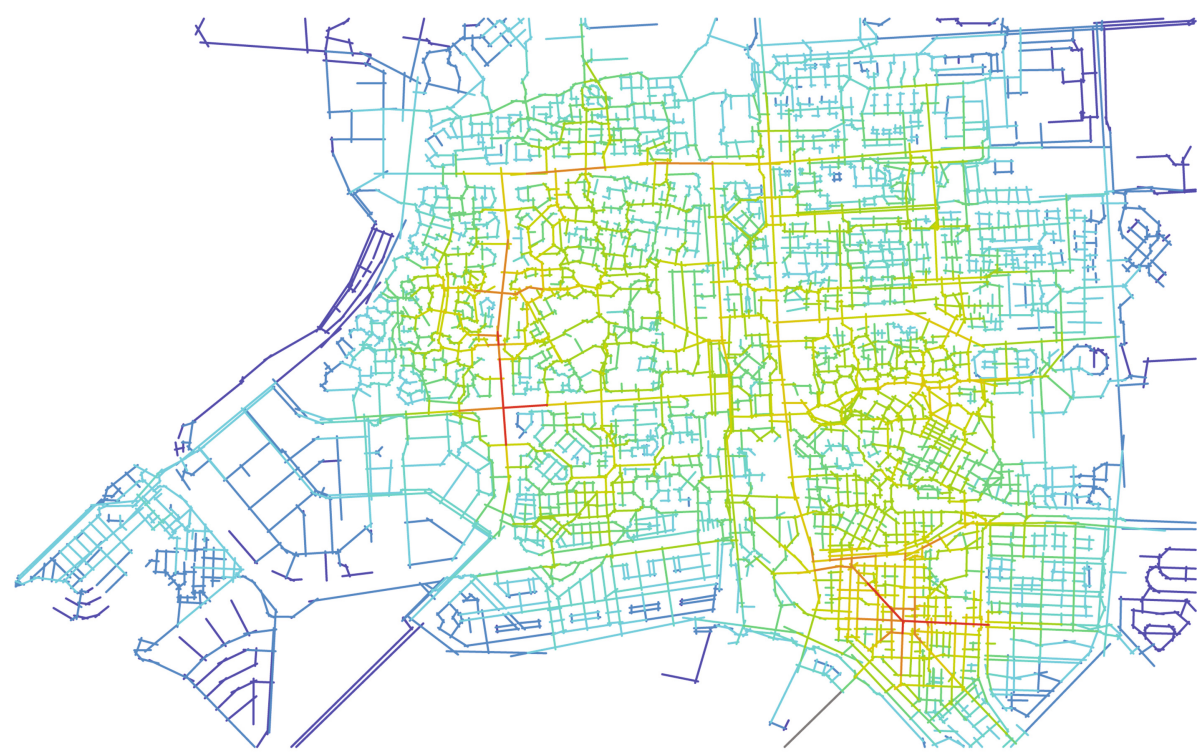

(b) Lelystad: Angular (segment) integration, radius $600 \mathrm{~m}$

Fig. 2.41 Angular (segment) integration analysis for the town of Lelystad with (a) a radius of 6,000 m and (b) a radius of 600 metres

Again, we use the Lelystad and Hilversum cases to show how various metric radii highlight the various to-movement potentials on different scale levels. Figure 2.41 shows an angular (segment) integration analysis with a high metric radius of $6,000 \mathrm{~m}$ and a low metric radius of $600 \mathrm{~m}$ for Lelystad. The to-movement potentials on a citywide scale are in a different location than on a neighbourhood scale. The main shopping centre is located along the streets with the highest values for the angular (segment) integration analysis with a radius of $600 \mathrm{~m}$ (a) and outlet shops, furniture shops, and car dealers are located along the roads with the highest numerical values for the angular (segment) integration analysis with a radius of $600 \mathrm{~m}$ (b).

Conversely, in Hilversum the streets with high numerical values for the high metric and low metric analyses overlap in the city centre (Fig. 2.42). Large and small enterprises are located in the city centre and along the integrated main streets leading to the city centre. Hilversum offers a large variation in the types of shops in comparison with Lelystad. 


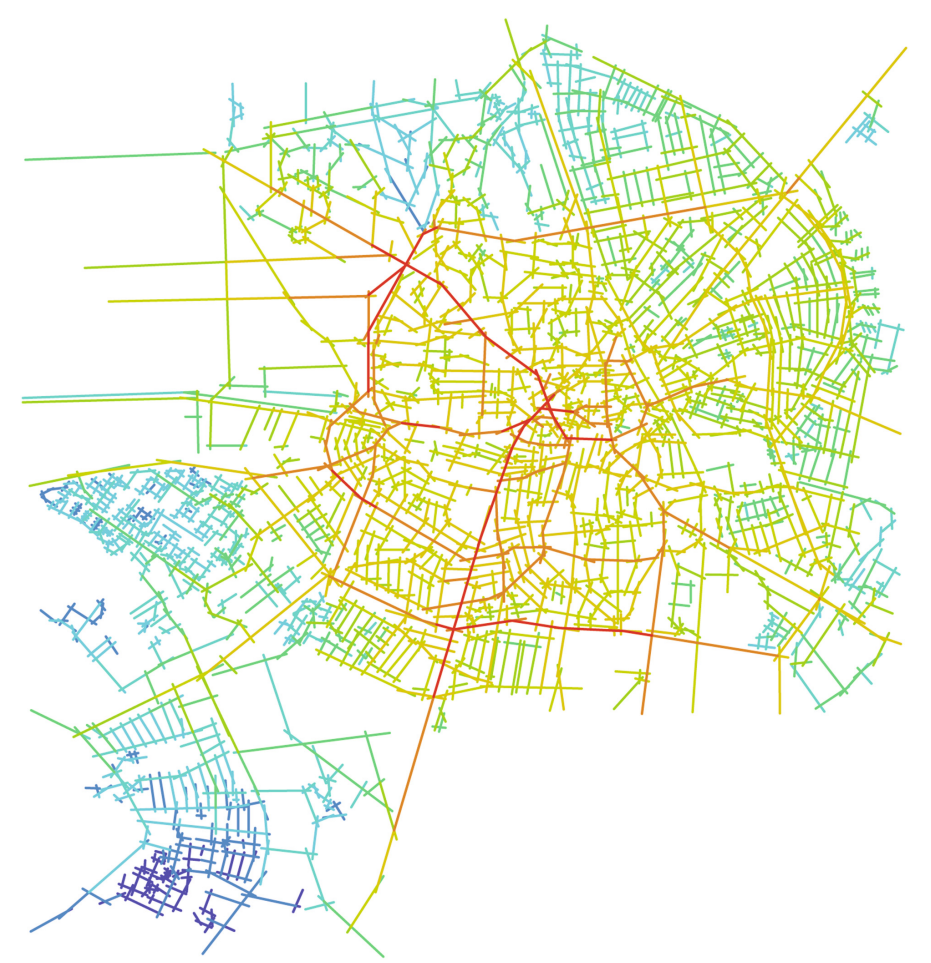

(a) Hilversum: Angular (segment) integration, radius $6000 \mathrm{~m}$

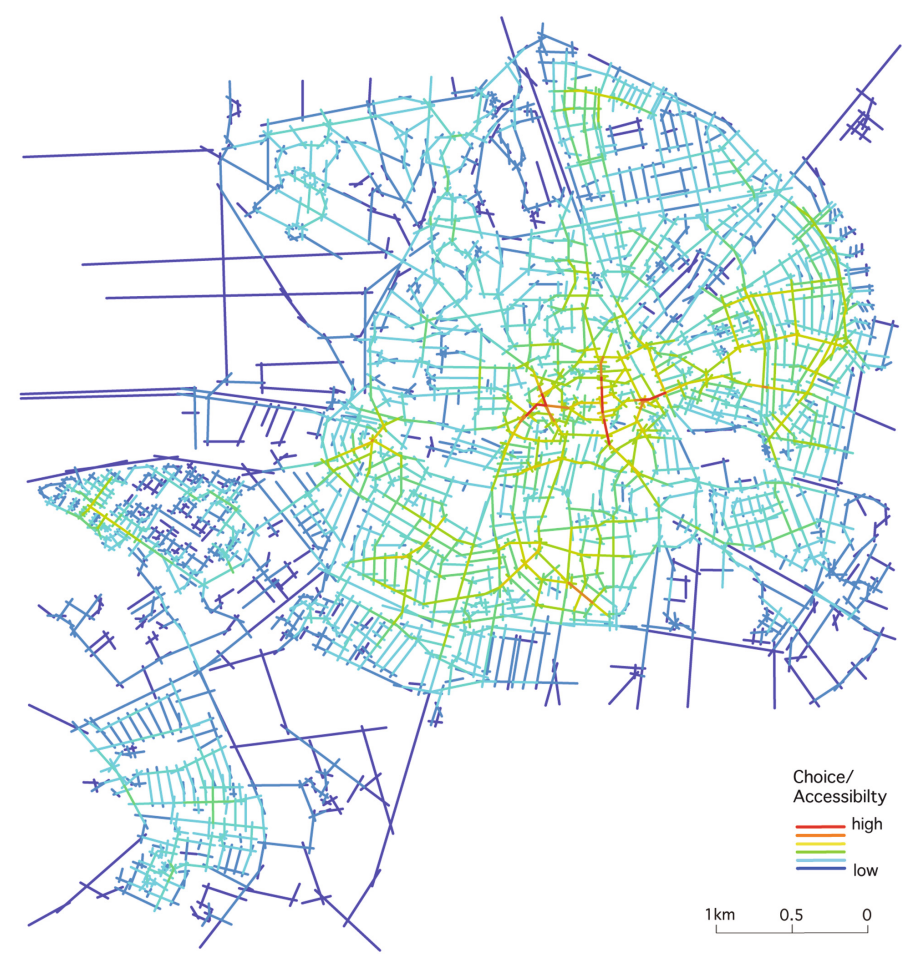

(b) Hilversum: Angular (segment) integration, radius 600m

Fig. 2.42 Angular (segment) integration analysis for the town of Hilversum with (a) a high metric radius of 6,000 $\mathrm{m}$ and (b) a low metric radius of $600 \mathrm{~m}$

For an in-depth understanding, and if you like mathematics, the next chapter explains more elaborately how to calculate angular choice. For the less-interested readers, we suggest moving on to reading Sect. 2.5.4 "Normalising the angular integration and choice values: NACH and NAIN". 


\subsubsection{A More Elaborate Explanation for How to Calculate Angular (Segment) Integration and Choice}

The basis of the space syntax method is the axial map, and the basic premise is to calculate the topological depth from one axis to all others, in other words, the total number of direction changes from one axis to all others. The integration $(I)$ of an axial line $(i)$ is a function of its depth related to all other axes. The calculation behind the integration of an axial line is (Rashid 2017 p. 64):

$$
l_{i}=\frac{2\left(n\left(\log _{2}\left(\frac{n+2}{3}\right)-1\right)+1\right) /(n-1)(n-2)}{2\left(\left(\frac{\sum_{j=1}^{n} d_{i j}}{n-1}\right)-1\right) /(n-2)}
$$

where $n$ is the number of segments and $d_{i j}$ is the shortest distance (least number of direction changes) between two segments $i$ and $j$. The greater the number of steps $\left(d_{i j}\right)$ between street axes, the lower the integration values become. The choice of a street axis shows how likely one is to pass through that axis when moving around in a built environment. Choice measures the degree of betweenness and measures the through-movement potentials. The formula of choice (C) of a street axis $(i)$ is as follows (Rashid 2017, p. 64):

$$
C_{i}=\sum_{J} \sum_{K} g_{j k}^{(i) \lg (j<k)}
$$

where $g_{j k}^{(i)}$ is the number of shortest paths between segment $j$ and $k$ containing $i$, and $g_{j k}$ is the number of all shortest paths between $j$ and $k$ (Rashid 2017, p. 64).

In the segment analysis, the axial lines are broken up where they cross each other (D'Acci 2019) or at each junction. The segment integration of a street shows how easy it is to get to that segment from all other segments. It calculates the to-movement potentials. Segment integration can be compared across systems. It measures how close each segment is to all others in terms of the sum of angular changes that are made on each route (Hillier and Iida 2005, pp. 475-490). Here, too, a radius of $500 \mathrm{~m}$ is taken for the local scale, and a radius of 5,000 $\mathrm{m}$ is used for the city scale. The choice takes the angular deviation of each topological step into account.

a)

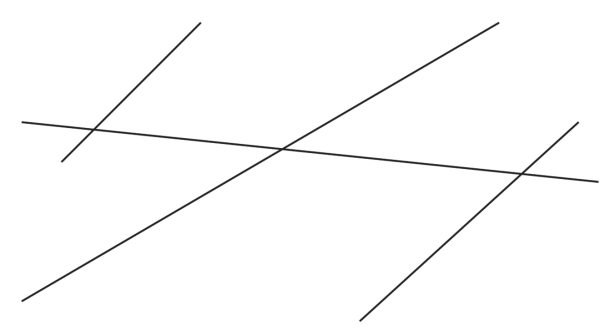

b)

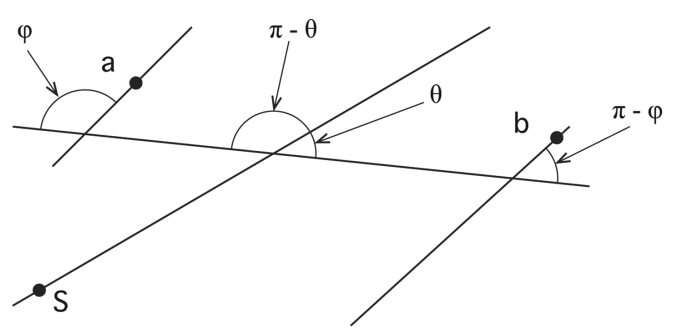

c)

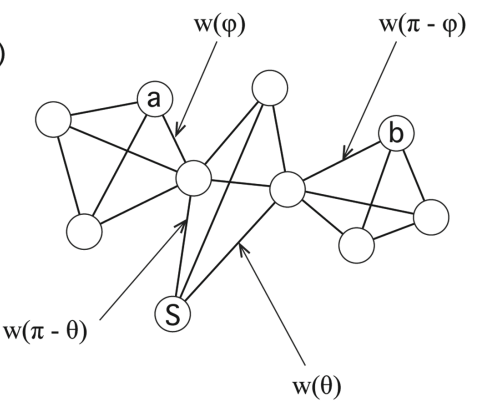

Fig. 2.43 It shows an axial map (a) and how a segment map is made (b), and $\mathbf{c}$ shows the representation of the spatial interrelationships of the segments in an unjustified graph Hillier, Bill (Image redrawn from Hillier and Iida 2005, p. 559) 
Figure 2.43b illustrates how the segment network is disaggregated at the intersections of axial lines. The distance is measured by taking the 'shortest path' from every line to all other lines. The angle between segments is now taken into account. The calculation between node $S$ to node $a$ is thus $(\pi-\theta)+\mathrm{w}(\varphi)$. As Hillier and Iida write,

\footnotetext{
The following weight definitions are used to represent different notions of distance: Least length (metric): The distance cost of routes is measured as the sum of segment lengths, defining length as the metric distance along the lines between the mid-points of two adjacent segments. The distance of two adjacent line segments is thus calculated as half the sum of their lengths. Fewest turns (topological): Distance cost is measured as the number of changes of direction that have to be taken on a route. In the example shown in Fig. $2.43 \mathrm{~b}, \mathrm{c}, \mathrm{w}(\theta)=\mathrm{w}$ $(\pi-\theta)=\mathrm{w}(\theta)=\mathrm{w}(\pi-\theta)=1$ (however, $\mathrm{w}(0)=0)$. Least angle change (geometric): Distance cost is measured as the sum of the angular changes that are made on a route by assigning a weight to each intersection proportionate to the angle of incidence of two line segments at the intersection. The weight is defined so that the distance gain will be 1 when the turn is a right angle. In other words, 'w $(\theta) \propto \theta$, $(0 \leq \theta<\pi, \mathrm{w}(0)=0, \mathrm{w}(\pi / 2)=1$ '” (Hillier and Iida 2005, p. 559).
}

The angular segment choice is calculated by counting the number of times each street segment falls on the shortest path between all pairs of segments within a selected distance (termed the 'radius'). The 'shortest path' refers to the path of least angular deviation (namely, the straightest route) through the system (Hillier and Iida 2005, p. 475). The angular integration of a segment $x$ is

$$
A I_{X}=\frac{1}{n} \sum_{i=1}^{n} d_{\theta}(\pi X, i)
$$

where $n$ is the number of segments and $d \theta$ is the angle between any two segments on the shortest path on segment $x$ (Rashid 2017 , p. 66). When adding the length $l$ of the segments, we get the following formula for the angular integration of a street segment with segment length:

$$
A I_{X}^{l}=\frac{\sum_{i=1}^{n} d_{\theta}(X, i) l(i)}{\sum_{i=1}^{n} l(i)}
$$

The issue we are dealing with is to calculate how easy it is to get to a street and how likely one is to pass through a street (D’Acci 2019) within certain metric radiuses.

\subsubsection{Normalising Angular Integration and Angular Choice Values: NAIN and NACH}

The first generated axial maps were not georeferenced because earlier versions of the space syntax computer application dealt only with topological distances. When the metric radius was introduced, the Depthmap software generated a unit system when loaded into the computer application for maps that are not georeferenced. For the georeferenced maps, the software identifies the real metric units.

Since 2012, normalised angular integration (NAIN) and normalised angular choice (NACH) have been applied (Hillier et al. 2012). At present, the computer application Depthmap allows normalising angular segment choice and angular segment integration analyses with metric radii directly in the software after processing the graph. Figure 2.44 depicts (a) a $\mathrm{NACH}$ analysis with global radius $\mathrm{n}$ and (b) a NAIN analysis with global radius $\mathrm{n}$ for Greater Vienna. 
Fig. 2.44 Normalised values from the angular segment analysis: (a) $\mathrm{NACH}$ radius $\mathrm{n}$ and (b) NAIN radius $\mathrm{n}$ for Greater Vienna

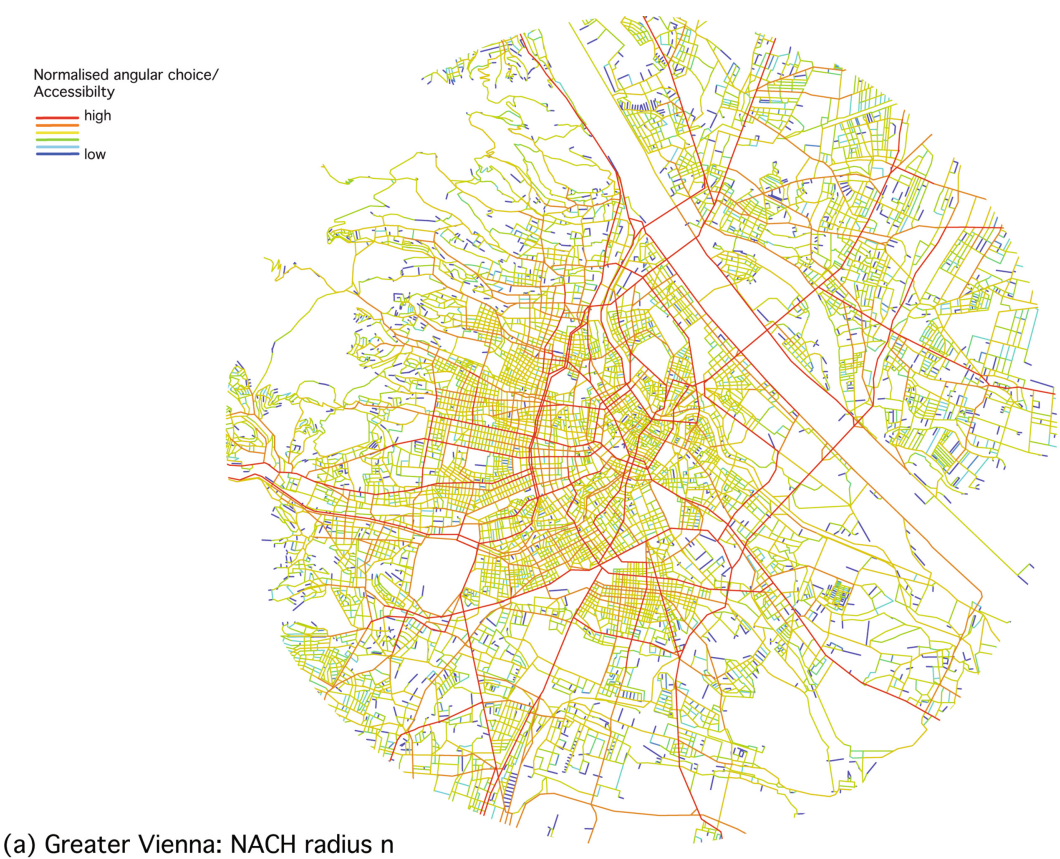

(a) Greater Vienna: NACH radius n

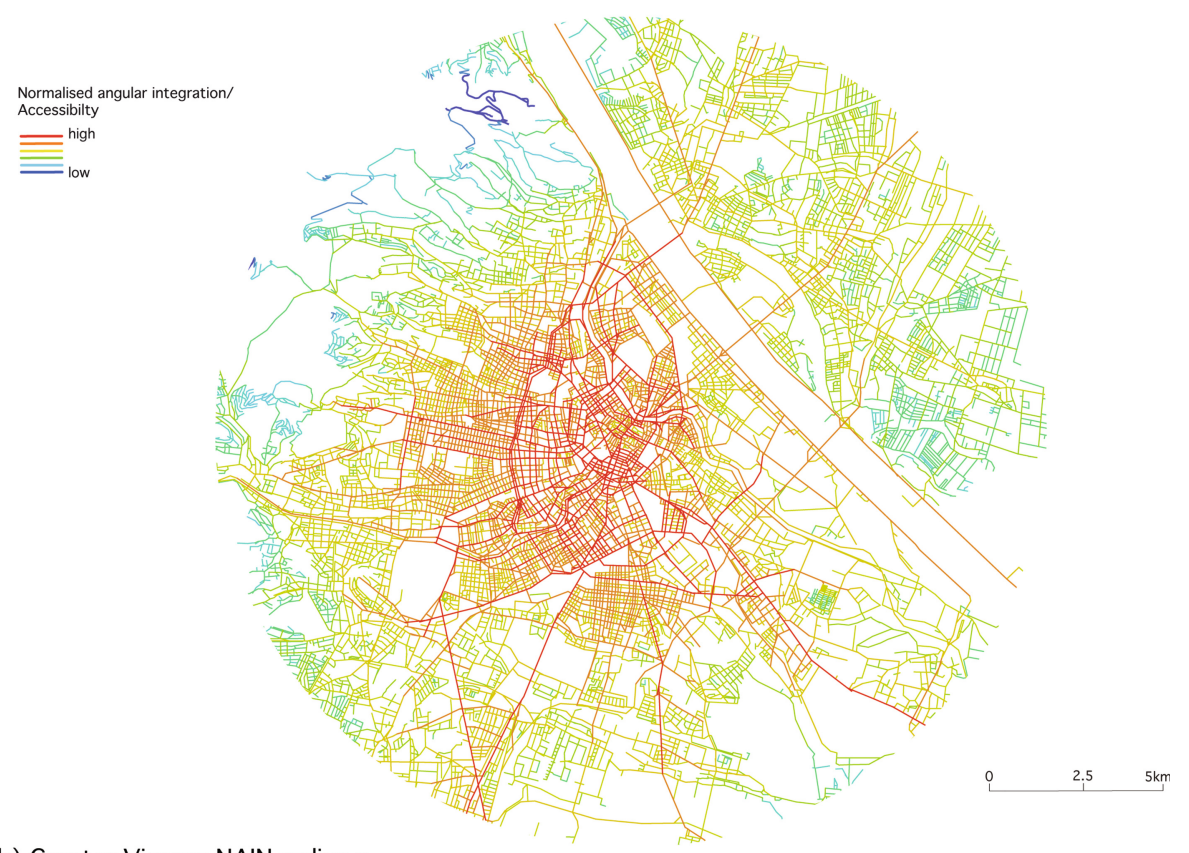

(b) Greater Vienna: NAIN radius $\mathrm{n}$

NAIN shows the to-movement potentials of various scale levels, whereas NACH shows the through-movement potentials. Economic centres tend to locate themselves in areas with streets that have high NAIN values, and preferably along streets with high $\mathrm{NACH}$ values for catching the through travellers.

$\mathrm{NACH}$ radius $\mathrm{n}$ depicts the deformed wheel pattern of the main route network. As can be seen in the NAIN radius $\mathrm{n}$ analysis, the main centre of Vienna is the historic core extended by the inner-city districts as being highly central (Fig. 2.44).

In contrast, the NACH radius $1,600 \mathrm{~m}$ highlights the most vital main route network on a local neighbourhood level. The NAIN radius $1,600 \mathrm{~m}$ shows the to-movement potentials for various neighbourhoods. All of these local centres tend to have a very high pedestrian inter-accessibility (Fig. 2.45). 
Fig. 2.45 Normalised values from the angular segment analysis: (a) NACH radius $1,600 \mathrm{~m}$ and (b) NAIN 1,600 m for Greater Vienna

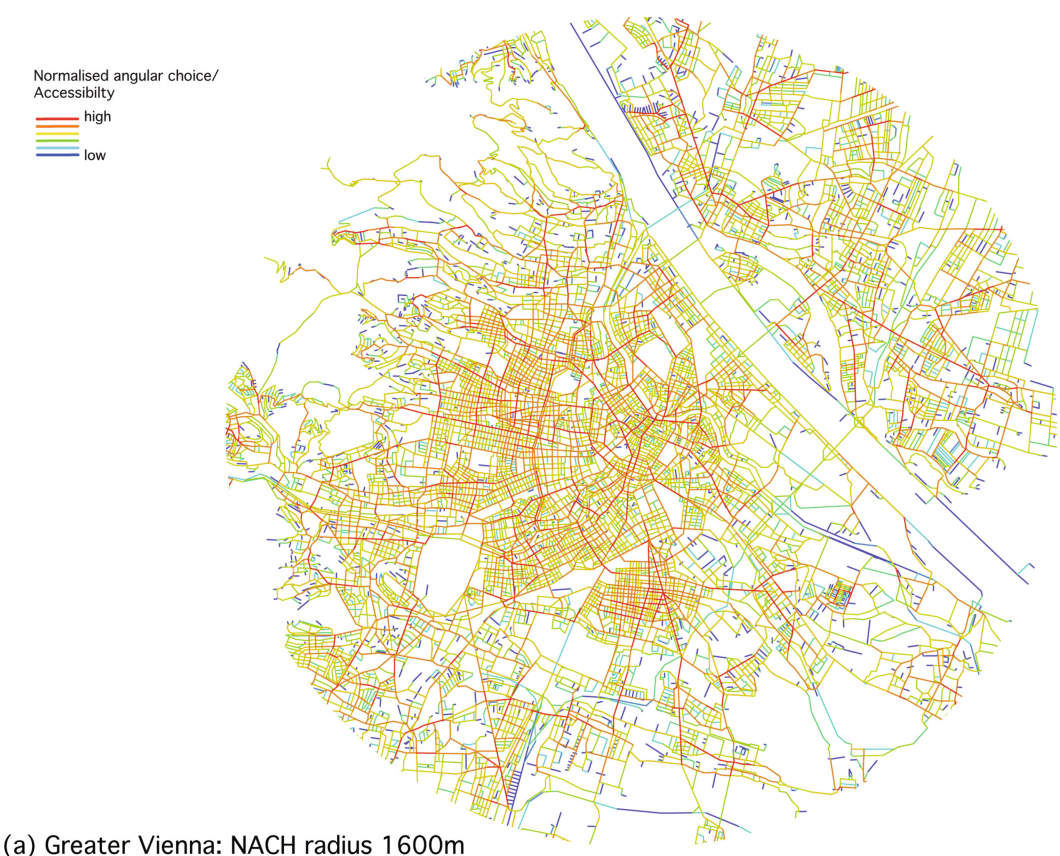

(a) Greater Vienna: $\mathrm{NACH}$ radius $1600 \mathrm{~m}$

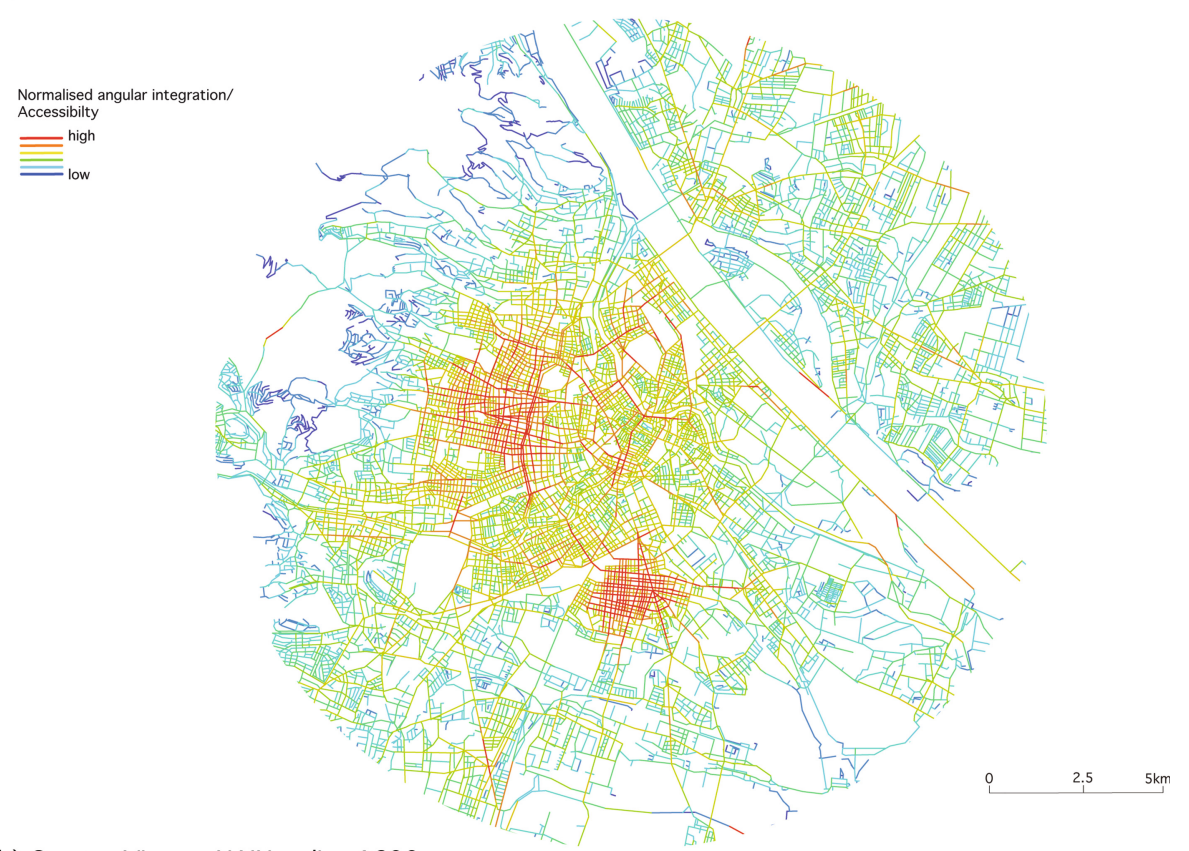

(b) Greater Vienna: NAIN radius $1600 \mathrm{~m}$

For normalising the values, the following equations can be best used for NACH and NAIN for a radius r:

$$
\begin{aligned}
& \text { NACH }=\log (\text { Choice }(r)+2) \\
& \text { NAIN }=\log (\text { Integration }(r)+2)
\end{aligned}
$$

These normalisation formulas are robust and work well for all types of built environments. The usefulness of NAIN and $\mathrm{NACH}$ is when comparing different cities with one another, in particular when the axial maps are not georeferenced. The numerical values of the NAIN and NACH analyses of Vienna in Figs. 2.44 and 2.45 were normalised with these two mathematical formulae. When all the maps are georeferenced with the same type of units, there is in principle no need to normalise the values.

Currently, there are experiments going on to develop more advanced normalisation formulas for NACH and NAIN. Both radius $\mathrm{n}$ and various local metric radii can be applied (Hillier et al. 2012, p. 191): 


$$
\begin{gathered}
\mathrm{NACH}=\frac{\log (\text { Choice }(\mathrm{r})+1)}{\log (\text { Total depth }(\mathrm{r})+3)} \\
\text { NAIN }=\frac{1.2 \sqrt{\text { Node count }(\mathrm{r})}}{\text { Total depth }(\mathrm{r})+2}
\end{gathered}
$$

Both equations were developed and tested through the comparison of 50 different cities and large neighbourhoods. The chosen cities had a large variation in geographical location, cultural background, and spatial layout (from strict orthogonal to an organic labyrinthine network) and size (from less than 1,000 up to 250,000 segments) (Hillier et al. 2012).

The following cities were chosen:

(1) USA: Manhattan, Chicago, Chicago centre, Denver, Charleston, Las Vegas, Atlanta, New Orleans, Hollywood, and Washington;

(2) South and Central America: Santiago, Mexico City (central areas), Rio de Janeiro, Brasilia, Recife, Teotihuacan, Uberlandia, Sao Paulo, Petropolis, and Ouro Preto;

(3) Europe: Barcelona, Mytilene, Nicosia, Nicosia in walls, Venice, Athens, Hamburg, Canterbury, Munich, Antwerp, London, Madrid, Alkmaar, Amsterdam, Rome, Bath, Apt, and Gouda;

(4) Middle East: Hamedan, Shiraz, Istanbul, Jeddah, Ahmedabad, and Konya;

(5) Asia: Tokyo, Beijing, Kyoto, Suzhou, Hong Kong, Xangai, and Shanghai;

(6) Australia and New Zealand: Auckland.

In addition, Hillier et al. (2012) tested four different neighbourhoods in London as reference cases: Clerkenwell, Barnsbury, Brompton, and South Kensington. The reason for this choice was that dense movement has been observed in these areas. The collected empirical movement data were tested against various spatial calculations. The two different normalisation equations for angular segment integration and angular choice seem to work well for a cross-comparison of different cities and urban areas.

\subsection{Four-Pointed Star Model}

In 2012, Bill Hillier and his colleagues experimented with combining various normalised measurements, such as combining the mean NACH or mean NAIN with the max NAIN or max NACH (Hillier et al. 2012, p. 170). For comparing the 50 cities with each other, they applied these four measures in a four-pointed star model by using Z-scores or standard scores. The $Z$-score is a value's relationship to the average of a group of values, measured in terms of standard deviations from the mean. A positive Z-score indicates that the value is above the mean, and a negative Z-score indicates that the value is below the mean. In this way, Bill Hillier, Tao Yang, and Alasdair Turner made it possible to compare cities with one another for describing the degree of integration of their foreground networks in relation to their background networks.

As the results show, various cultures influence the relationship between the background and the foreground network. Arab and Persian cities have low values for the correlations between these four measures, whereas Greek cities have high values. Chinese cities have a highly integrated foreground network, but a poorly integrated background network. European cities vary a lot on these scales. The essence is that the foreground network (identified by the NACH analysis) is needed to interconnect the various centres (identified by the NAIN analysis) in a city with one another.

For generating the four-pointed star model, the following equations for calculating the Z-scores are applied for both the NAIN and NACH values:

$$
\begin{aligned}
\bar{X}_{\max } & =\frac{\sum_{i=1}^{50} X_{\max }(i)}{50} \\
Z_{\max }(i) & =\frac{X_{\max }(i)-\bar{X}_{\max }}{\bar{S}_{\max }(i)}
\end{aligned}
$$

where $X_{\max }(i)$ denotes the maximum value of the city $i$.

$$
\begin{aligned}
\bar{X}_{\text {mean }} & =\frac{\sum_{i=1}^{50} X_{\text {mean }}(i)}{50} \\
Z_{\text {mean }}(i) & =\frac{X_{\text {mean }}(i)-\bar{X}_{\text {mean }}}{\bar{S}_{\text {mean }}(i)}
\end{aligned}
$$


Table 2.1 Values from the 50 cities analysed by Hillier et al. (2012)

\begin{tabular}{l|l|l|l}
\hline & NACH & NAIN \\
\hline$X_{\max }$ & 1.5679 & 1.8705 \\
\hline$X_{\text {mean }}$ & 0.912 & 1.2206 \\
\hline $\mathrm{S}\left(X_{\text {mean }}\right)$ & 0.098 & 0.522 \\
\hline $\mathrm{S}\left(X_{\max }\right)$ & 0.0669 & 0.767
\end{tabular}

where $X_{\text {mean }}(i)$ denotes the mean value of the city $i$. where $S$ is the standard deviation from the 50 cities, $x_{\max }$ is the maximum value, and $x_{\text {mean }}$ is the average value for NACH or NAIN either from a city under scrutiny or from the 50 cities analysed by Hillier et al., (2012). Further, they compared the maximum NAIN and NACH as well as the mean NAIN and NACH of these cities. Because the 50 cities are representatives from America, Europe, the Middle and Far East, and Australia/New Zealand, the following values can be used based on these 50 cities (Hillier et al. 2012, pp. 164ff). Table 2.1 shows the various max and mean values from the 50 cities analysed by Hillier et al. (2012).

The sizes of the chosen 50 cities did not matter, and all of the radius $\mathrm{n}$ measures could be used (Hillier et al. 2012). Therefore, their mean values can be applied to other cities with the purpose of testing to what extent a particular city scores above or below the average in terms of to-movement (NAIN) or through movement (NACH) . The mean values represent the majority of streets in the city, which is the background network. Conversely, the maximum values represent the main route network, which is the foreground network. NAIN represents the to-movement potential where the max values often represent the main shopping streets, and NACH represents the through-movement potential, where the maximum values represent the main routes through or between various urban areas.

In the following, we will apply these mean values from the 50 cities for the four-pointed star model to our examples of the towns of Lelystad and Hilversum and the city of Vienna. In the Depthmap software, the column properties allow one to retrieve the max and mean values for NACH and NAIN.

For the Dutch town of Hilversum:

NAIN max $=1.4046$
NAIN mean $=0.951158$
NACH max $=1.56825$
NACH mean $=0.603626$

$X_{\text {mean }}$ and the standard deviation values $S$ are used from the comparison of the 50 cities. Thus, we get the following results:

$$
\begin{gathered}
Z\left(N A I N_{\text {max }}\right)=\frac{(1.4045-1.8705)}{0.767}=-0.608 \\
Z\left(N A I N_{\text {mean }}\right)=\frac{(0.951158-1.2206)}{0.522}=-1.3871 \\
Z\left(N A C H_{\text {max }}\right)=\frac{(1.56825-1.5679)}{0.0669}=0.0052 \\
Z\left(N A C H_{\text {mean }}\right)=\frac{(0.0603626-0.912)}{0.098}=-3.147
\end{gathered}
$$

We applied the same logic to the town of Lelystad:

NAIN max $=1.56903$

NAIN mean $=0.901995$

$\mathrm{NACH} \max =1.65309$

$\mathrm{NACH}$ mean $=0.552625$

$$
Z\left(N A I N_{\max }\right)=\frac{(1.56903-1.8705)}{0.767}=-0.8697
$$




$$
\begin{gathered}
Z\left(N A I N_{\text {mean }}\right)=\frac{(0.901995-1.2206)}{0.522}=-0.6104 \\
Z\left(N A C H_{\text {max }}\right)=\frac{(1.65309-1.5679)}{0.0669}=1.2734 \\
Z\left(N A C H_{\text {mean }}\right)=\frac{(0.552625-0.912)}{0.098}=-3.6671
\end{gathered}
$$

Figure 2.46 shows a four-pointed star model of Hilversum and Lelystad. Each measure is a standard score (Z-score) varying about 0 with a negative minimum at the centre and positive maximum at the edge (Hillier et al 2012, p. 170). On the $\mathrm{x}$-axis, we plot the mean NACH (top) and mean NAIN (bottom) values, and on the y-axis we plot the max NAIN (left) and max NACH (right). The four-pointed star diagrams for Hilversum and Lelystad depict how they spatially behave and score in comparison and in relation to the average of the 50 cities and how their values deviate from this average.

Fig. 2.46 Four-pointed star model of Hilversum and Lelystad

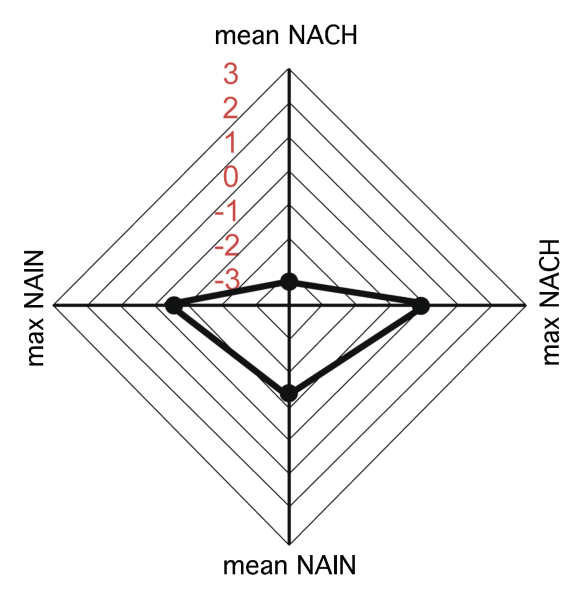

Hilversum

Max NAIN: 1.4045

Mean NAIN: 0.951158

Z-score for max NAIN: -0.608

Z-score for mean NAIN: -1.3871

Max NACH: 1.56825

Mean NACH: 0.603626

Z-score for max NACH: 0.0052

Z-score for mean NACH: -3.147

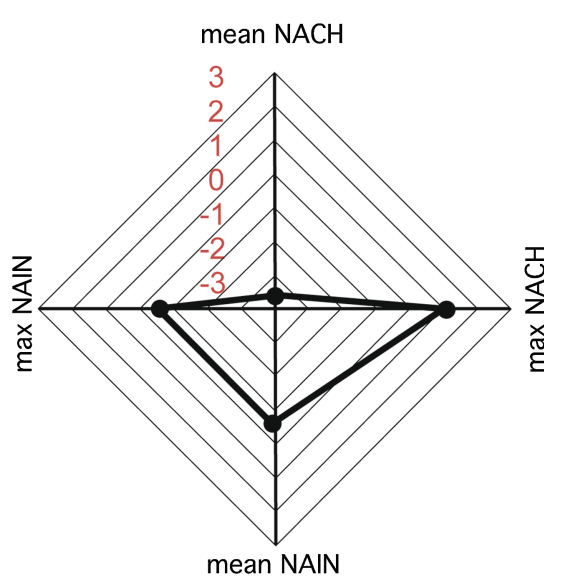

Lelystad

Max NAIN: 1.56903

Mean NAIN: 0.901995

Z-score for max NAIN: -0.8697

Z-score for mean NAIN: -0.6104

Max NACH: 1.65309

Mean NACH: 0.552625

Z-score for max NACH: 1.2734

Z-score for mean NACH: -3.6671

From the results for both Hilversum and Lelystad in relation to the 50 cities, we can see that the to-movement potentials (NAIN) score slightly below average for both the maximum and minimum values. For the through-movement potentials, both cities also score very low on the NACH mean values, which represents the background network. For the maximum $\mathrm{NACH}$ value, Lelystad is above average for the main route network, whereas Hilversum is close to the average. These results tell us first of all that Hilversum and Lelystad have very different spatial setups. Both town' background networks are highly segregated. Lelystad's strong foreground network suggests that all segregated neighbourhoods are held together by the main route network, which supports car-based movement.

Even though Hilversum and Lelystad have very different spatial patterns-new town versus historic town - the differences between them in relation to the 50 cities is not large. The local Dutch culture has influenced the spatial structure of the background network in the modern planned cities. This means that within neighbourhoods, the through-movement potentials are very low. All segregated neighbourhoods are connected to each other with a highly integrated main route network, mainly for vehicular movement.

Interestingly, Greater Vienna's four-pointed star model depicts a different spatial reality. All values are close to the average in comparison to the 50 cities. For the NACH values, the main route network has average integration values and is connected to the whole region around Vienna. For the NAIN values, the scattered former towns and villages around Greater 
Vienna lower the NAIN values. If just Vienna's historic core is analysed separately, the NAIN values would be high. However, most towns and villages are well connected to the main route network. In other words, Greater Vienna has a balance between its foreground and background networks for through-movement and to-movement. This means that spatially Vienna's neighbourhoods are very pedestrian-friendly and well-connected to the foreground network. Vienna is known to be a city with a high quality of life (Mercer Quality of Living Survey, 2019). Seemingly, Vienna's high scores for quality of life are also spatially supported (Yamu 2020) (Fig. 2.47).

Fig. 2.47 Four-pointed star model of Greater Vienna

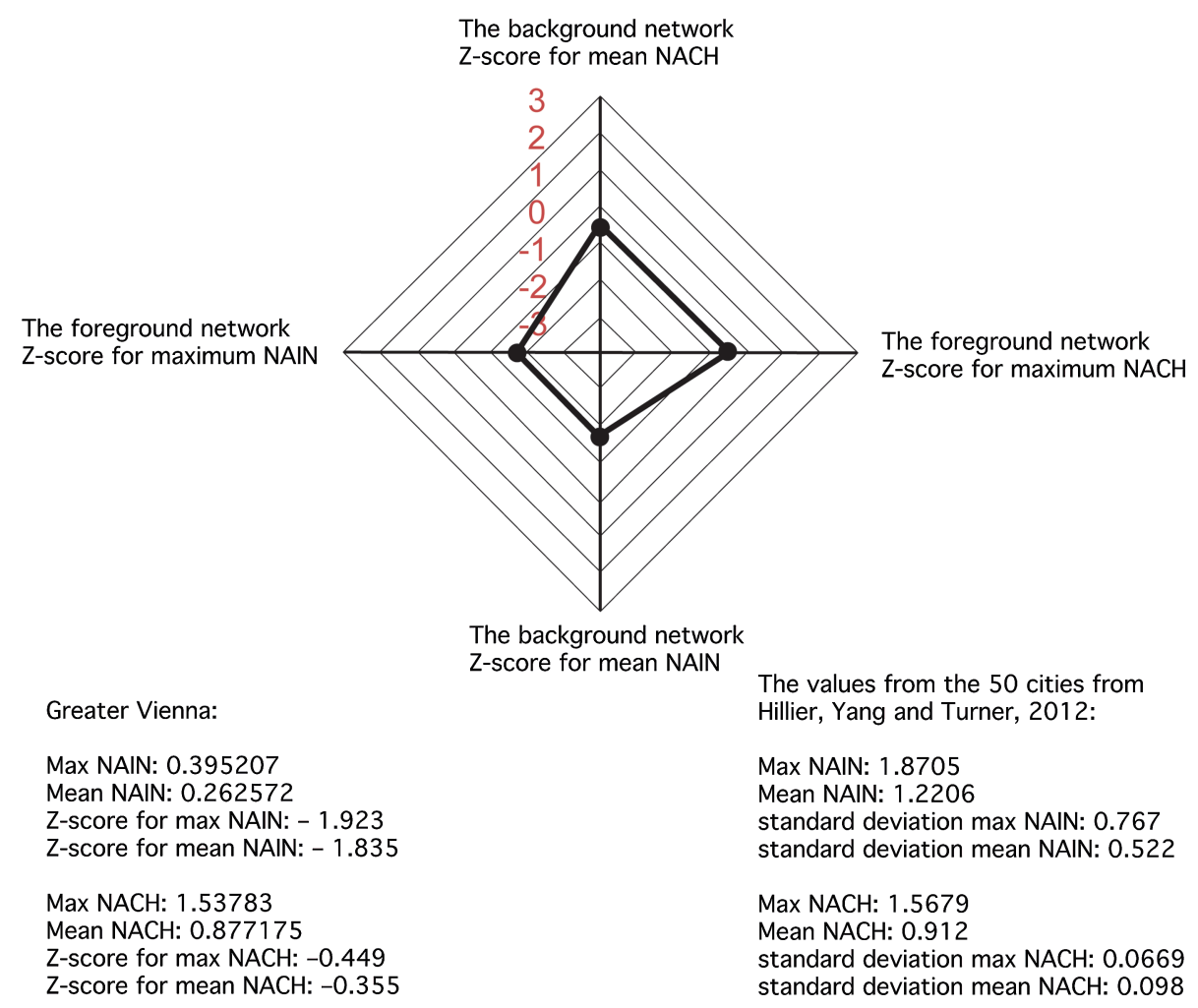

\subsection{The Use of Scatterplots}

Scatterplots are useful for identifying the type of relationship between two quantitative variables. For example, correlations between global and local integration values refer to the concept of the 'intelligibility' of an urban system. The closer the correlation coefficient is to one, the more orientable and thus intelligible the built environment under scrutiny is. A high number on the correlation coefficient means that when staying in a local area, the street pattern logic of a whole city can be understood. For example, when standing in a neighbourhood with a strict orthogonal street network, it can be easily understood that the whole city follows a similar logic, for example, Manhattan in New York. Thus, the concept of intelligibility also refers to cognitive mapping and to Lynch's approach (1960).

Scatterplots are also useful when correlating angular choice results with high and low metric radii because this can indicate the degree of street life in built environments. When the correlation coefficient is high, the locally integrated main routes are also highly integrated on a city scale. Conversely, when the correlation coefficient is low, the locally integrated main routes are located far away from the main routes on a city scale. Figure 2.48 shows the scatterplots for Lelystad and Hilversum. The correlation coefficient $\left(R^{2}\right)$ for Lelystad is very low in comparison with Hilversum. In Hilversum centre, the streets have high values for angular choice analyses with both high and low metric radii, whereas the streets in Lelystad have high values either on a local or on a city scale. 

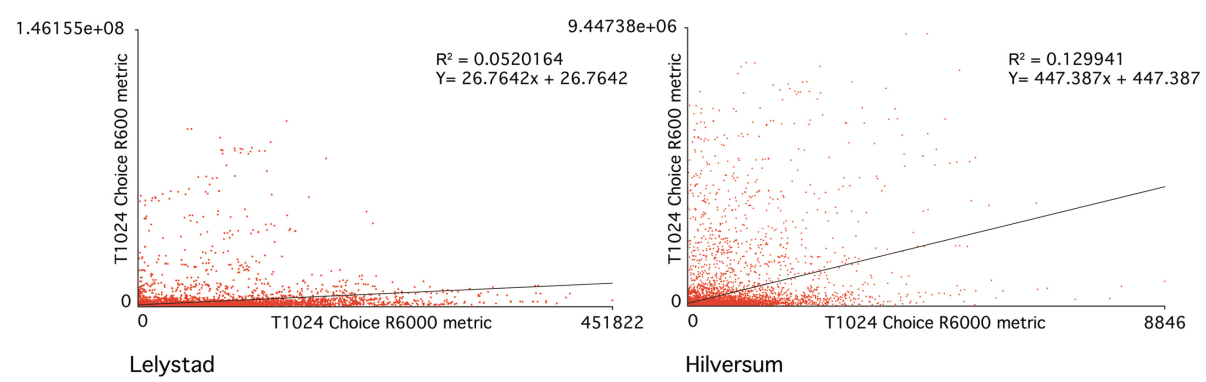

Fig. 2.48 Scatterplots for Lelystand (left) and Hilversum (right)

\subsection{Conclusions: Common Errors and Mistakes When Analysing Linear Urban Space}

Axial and segment analyses have undergone great changes since the 1990s. Software developments, computer capacity, access to big data over time, and criticism of weaknesses of earlier methods have contributed to methodological and analytical developments. Old axial analysis calculation models from the 1990s, such as radius-radius integration analysis and integration gradient analysis have been stepping-stones on the way forward for the constant improvement of the current calculation methods (Yamu et al. 2021). Over the past decade, the accuracy of the results from the segment-based angular choice and angular integration analyses with various metric radii has been established through the application of these measurements in research and consultancy wordwide.

However, a typical beginner's error when drawing an axial map is that the axial lines are not properly linked with each other. Often, a subset of axial lines is not connected to an axial line from the overall axial map. This can distort the results of the analysis on a citywide and local scale. Another beginner's error is that the axial map is drawn with polylines instead of single lines. A polyline axial map makes it difficult to run a depth analysis from a particular line. When some road or street links are missing in the axial map, this leads to incorrect conclusions from the space syntax analyses. The same accounts for forgetting to unlink axial lines representing bridges and tunnels. Wrong connections of axes and segments can distort the computed results. Furthermore, beginners tend to 'over-model', drawing too many axial lines for producing an axial map. By definition, an axial map is the minimal set of axial lines representing sightlines indicating movement paths. Practice is needed to produce an axial map with the minimal set of axial lines.

Another typical misuse of space syntax is to include light rail or metro lines in the axial map. Public transport travellers cannot make a decision to make a turn to a side street when travelling between public transport stops. Therefore, rails cannot be included. The best is to analyse the results from the space syntax analyses at the public transport stops. Where the results from the choice and integration analyses are high at all scale levels, there is a greater need for public transport facilities.

First of all, focus on the research question when making an axial map. When investigating pedestrian accessibility or anti-social behaviour in public spaces, the pedestrian movement routes on pavements, subways, paths through parks, etc. need to be represented as axial lines. Here, the highway network lacking pavements can be left out. When investigating larger metropolitan areas, the highway network needs to be added to the axial map. At present, the challenge is to develop well-functioning application software for generating axial maps from road centre lines in geographic information systems (GIS). Manually generating axial maps of large metropolitan areas is a monotonous and time-consuming task to undertake.

Another new beginner error can be the incorrect interpretation of the spatial analysis results. Therefore, we advise first drawing and analysing a neighbourhood or town you are familiar with. We suggest you to read the theory chapter and some of other literature related to your research topic on space and society.

\subsection{Exercises}

For most of the exercises, please use software. DepthmapX and "DepthmapX manual for dummies" can be downloaded from www.spacesyntax.net and www.github.com.

\section{Exercise 1}

Carry out a manual one-step analysis from the main routes from two different urban areas from a city or town. 


\section{Exercise 2}

Carry out a manual two-step analysis from a shopping street and a residential street from your own neighbourhood or a neighbourhood you are familiar with. Compare the total number of axes that can be reached within a radius of $500 \mathrm{~m}$ starting from both streets.

\section{Exercise 3}

Carry out a manual n-step analysis from the main route system of your own neighbourhood. What is the maximum number of direction changes from the main route network to the local streets of your neighbourhood? What kinds of functions are located in the buildings of these streets?

\section{Exercise 4}

Draw an axial map from two neighbourhoods or towns you know. You can use any software that allows you to export a dxf. file or mif. file. Best is to use any geographic information system software because it allows the generation of a georeferenced axial map. For comparison, you can draw two axial maps, one from a modern or newly developed neighbourhood and one from a historic neighbourhood. This allows for a better comparison. Process the map and compute the connectivity and the global and local axial integration using Depthmap software. Reflect upon the results. Where are the local centre(s) located? Are there any differences between connectivity and global and local integration results?

\section{Exercise 5}

Carry out a step depth analysis using the Depthmap software from all public transport stops in your neighbourhood. Describe the degree of accessibility to the neighbourhood for these public transport stops.

\section{Exercise 6}

Correlate in the Depthmap software the local and global integration results of the two neighbourhoods from exercise 4 . What is the value of the correlation coefficient? What is the degree of intelligibility of these neighbourhoods? What does this tell us about the neighbourhoods?

\section{Exercise 7}

Carry out angular integration and angular choice analyses with low and high metric radii of the neighbourhoods using the Depthmap software. Where are the main routes located? Are they going around or through the neighbourhoods? Describe the spatial features of the local centre(s) in these areas.

\section{Exercise 8}

Using the Depthmap software, correlate the angular choice values with high and low metric radii for both neighbourhoods. Describe and reflect upon the results.

\section{Exercise 9}

Normalise the obtained results from your angular integration and angular choice analyses for high and low metric radii. Generate a four-pointed star model for the neighbourhood using the values from Table 2.1. Explain and reflect upon the results.

\section{References}

Bhagwati, J. 2004. In defense of globalization. Oxford, New York: Oxford University Press.

Bruyns, G., D. Nel, C.D. Higgens, and A. van Nes. 2019. Flat versus volumetric methodologies. Restructuring spatial analysis and other indices. In Proceedings of the 12th international space syntax symposium, ed. S. Qiang et al. Beijing: Beijing Jiao Tong University.

van Casteren, J. Lelystad Prometheus, and R. Conroy-Dalton. 2008. The secret is to follow your nose. In Proceedings space syntax. 3rd international symposium, ed. J. Peponis, J. Wineman, and S. Bafna. Atlanta.

Czerkauer-Yamu, C., and A. Voigt. 2011. Strategic planning with space syntax. In Proceedings in eCAADe-29th conference in education in computer aided architectural design in Europe, 125-133. Ljubljana.

Czerkauer-Yamu, C. 2012. Strategic planning for the development of sustainable metropolitan areas using a multi-scale decision support system: The vienna case. PhD thesis, Université de Besançon/University alliance: Université de Franche-Comté.

D'Acci, L. 2019. Orientational versus esthetical urban street morphology parameterization in Space Syntax. Spatial Cognition \& Computation 19 (3). 
Dalton, N. Axman. 1998. http://www.spacesyntax.org/software/macbundle.asp.

Dalton, N. 2001. Fractional configurational analysis and a solution to the manhattan problem. In Proceedings space syntax. 3rd international symposium, ed. J. Peponis, J. Wineman, and S. Bafna. Atlanta.

Dalton, N. 2005. New measures for local fractional angular integration or towards general relitivisation in space syntax. In Proceedings space syntax. 5th international symposium, ed. A. van Nes. Delft.

Desyllas, J. 2000. The relationship between urban street configuration and office rent patterns in Berlin. PhD thesis, The Bartlett, University College London.

Fakhurrazi, R., and A. van Nes. (2012). Space and panic. The application of space syntax to understand the relationship between mortality rates and spatial configuration in banda aceh during the tsunami 2004. In Proceedings space syntax. 8th international symposium, ed. M. Greene. Santiago de Chile.

Figuradeiro, L., L.A. Figueiredo, and L. Amorim. 2005. Continuity lines in the axial system. In Proceedings space syntax. 5th international symposium, ed. A. van Nes. Delft.

Gil, J., T. Varoudis, K. Karimi, and A. Penn. 2015. The space syntax toolkit: Integrating depthmapX and exploratory spatial analysis workflows in QGIS. In Proceedings of the 10th international space syntax symposium. ed. K. Karimi, L. Vaughan, K. Sailer, G. Palaiologou, and T. Bolton. Space Syntax Laboratory, The Bartlett School of Architecture, UCL (University College London): London, UK. 2015.

Hillier, B. 1996. Space is the machine: A configurational theory of architecture. Cambridge, UK: Cambridge University Press.

Hillier, B. 1998. Against enclosure. In Rehu-manising housing, ed. N. Teymus, T. Markus, and T. Woaley, 63-85. London.: Butterworths.

Hillier, B., A. Penn, D. Banister, and J. Xu. 1998. Configurational modelling of urban movement network. Environment and Planning B: Planning and Design 25: 59-84.

Hillier, B. 1999. Centrality as a process: Accounting for attraction inequalities in deformed grids. Urban Design International 4 (3 \& 4): $107-127$.

Hillier, B. 2001. The theory of the city as object or how spatial laws mediate the social construction of urban space. In Proceedings space syntax. 3rd international symposium, ed. J. Peponis, J. Wineman, and S. Bafna. Atlanta.

Hillier, B. and J. Hanson. 1984. The social logic of space. Cambridge, UK: Cambridge University Press.

Hillier, B. 2005. A theory of human movement and its implications. In Presentation given at the opening session of the 5th international space syntax symposium.

Hillier, B., and S. Iida. 2005. Network effects and psychological effects: a theory of urban movement. In Proceedings space syntax. 5th international symposium, ed. A. van Nes. Delft.

Hillier, B., and Ö. Sahbaz. 2005. High resolution analysis of crime patterns in urban street networks: An initial statistical sketch from an ongoing study of a London borough. In Proceedings space syntax. 5th international symposium, ed. A. van Nes. Delft.

Hillier, B., A. Turner, T. Yang, and H.T. Park. 2007. Metric and topo-geometric properties of urban street networks. In Proceedings space syntax. 6th international symposium, ed. A.S. Kubat. Istanbul.

Hillier, B., T. Yang, and A. Turner. 2012. Normalising least angle choice in Depthmap and how it opens new perspectives on the global and local analysis of city space. Journal of Space Syntax 3 (2).

Humpert, K. 2007. Lauf-Spuren. Wien: Edition Esefeld \& Traub.

Jiang et al. 2000. Integration of space syntax into GIS for modelling urban spaces. International Journal of Applied Earth Obesrvation and Geoinformation 2 (3-4). Elsevier.

Joutsiniemi, A. 2005. The Ajax project: New theory and new software for space syntax. In Proceedings space syntax. 5th international symposium, ed. A. van Nes. Delft.

Karimi, K. 1998. Continuity and change in old cities; an analysis investigation of the spatial structure in Iranian and English historic cities before and after modernisation. PhD thesis, The Bartlett School of Graduate studies, University College London.

Krüger, M., and A.P. Vieira. 2012. Scaling relative asymmetry in space syntax analysis. Journal of Space Syntax 3 (2).

López, M., and A van Nes. 2007. Space and crime in Dutch built environments. Macro and micro spatial conditions for residential burglaries and thefts from cars. In Proceedings space syntax. 6th international symposium, ed. A.S. Kubat. Istanbul.

Lynch, K. 1960. The image of the city. Cambridge, MA: MIT Press.

Marcus, L. 2007. Spatial capital and how to measure it: An outline of an analytical theory of the social performativity of urban form. In Proceedings, sixth international space syntax symposium, ed. A. Kubat, E. Özhan, Y. Guney, and E. Eyuboglu. Istanbul: Istanbul Technical University.

Marshall, S. 2005. Streets and patterns. Spon Press, Oxon.

van Nes, A. 2005. Typology of shopping areas in Amsterdam. In Proceedings space syntax. 5th international symposium, ed. A. van Nes. Delft.

Van Nes, A. 2009. Analysing larger metropolitan areas on identification criteria for middle scale networks. In Proceedings 7 th International Space Syntax Symposium, ed. D. Koch, L. Markus, and J. Steen. Stockholm.

Van Nes, A., and C. Yamu. 2018. Space Syntax: a method to measure urban space related to social, economic and cognitive factors. In The Virtual and the Real in Planning and Urban Design: Perspectives, Practices and Applications, eds. C. Yamu, A. Poplin, O. Devisch,and G. De Roo (pp. 136-150). (Routledge Research in Planningand Urban Design). Routledge: Taylor and Francis group

Puckett, R.R. 2009. Multi-agent crowd behaviour simulation for tsunami evacuation, MSc thesis, Department of information and computer sciences of the University of Hawaii.

Rashid, M. 2017. The geometry of urban layouts. Springer.

Ratti, C. 2004. Space syntax: Some inconsistencies. Environment and Planning B: Planning and Design 31 (4): 487-499.

Sevtsuk A. 2010. Path and place: A study of urban geometry and retail activity in Cambridge and Somerville, MA, PhD dissertation in Urban Design and Planning, Massachusetts Institute of Technology.

Space Syntax Ltd. 2004. D3.1 Technology Platform Report. AGORA Cities for People.

Ståhle, A., L. Marcus, and A. Karlström. 2005. Place syntax-Geographic accessibility with axial lines in GIS. In Proceedings 5th international space syntax symposium, ed. A van Nes. TU Delft. Techne Press. Amsterdam.

Turner, A., A. Penn, and B. Hillier. 1993. An algorithmic definition of the axial map. Environment and Planning B: Planning and Design 32: 425444. 
Turner, A. 2000. Angular analysis: A method for the quantification of space. In CASA working paper series. Centre for Advanced Spatial Analysis, UCL: London.

Turner, A. 2001. Angular analysis. In Proceedings space syntax. 3rd international symposium, eds. J. Peponis, J. Wineman, and S. Bafna. Atlanta.

Turner A, 2005. Could a road-centre line be an axial line in disguise?, in Proceedings of the 5th International Symposium on Space Syntax, Volume 1 TU Delft, Delft, pp145-159

Turner, A. 2007. Ucl depthmap 7: From isovist analysis to generic spatial network analysis. New developments in space syntax software, ed. A. Turner. Istanbul.

Yamu, C. 2014. It is simply complex(ity). Modeling and simulation in the light of decision-making, emergent structures and a world of non-linearity. disP-The Planning Review 50 (4): 43-53.

Yamu, C. 2020. Emerging and shifting centralities: Evidence from Vienna. In Raumplanung. Jahrbuch 2020: 50Jahre Raumplanung an der TU Wien. eds. T. Dillinger, M. Getzner, A. Kanonier, and S. Zech, Studieren - Lehren - Forschen (pp. 542-559). (Jahrbücher des Instituts für Raumplanung der TU Wien; Vol. 8). NeuerWissenschaftlicher Verlag (NWV).

Yamu, C., and P. Frankhauser. 2015. Spatial accessibility to amenities, natural areas and urban green spaces: using a multiscale, multifractal simulation model formanaging urban sprawl. Environment and planning b-Planning \& design, 42(6): 1054. https://doi.org/10.1068/b130171p

Yamu, C., A. van Nes, and C. Garau. 2021. Bill Hillier's legacy: space syntax—a synopsis of basic concepts, measures, and empirical application. Sustainability, 13(6):3394. https://doi.org/10.3390/su13063394

Yamu, C., and A. van Nes. 2017. An integrated modeling approach. Combining multifractal urban planning with a space syntax perspective. Urban Science 1 (37). https://doi.org/10.3390/urbansci1040037

Yamu, C., and A. van Nes. 2019. Fractal urban models and their potential for sustainable mobility: A spatio-syntactic analysis. In Proceedings of the 12th InternationalSpace Syntax Symposium (pp. 415.01-415.13).

Yamu, C., G. de Roo, and P.Frankhauser. 2016. Assuming it is all about conditions: Framing a simulation model for complex, adaptive urban space. Environment andplanning b-Planning \& design, 43(6): 1019-1039. https://doi.org/10.1177/0265813515607858

Ye, Y., A, van Nes, Y. Ye, and A. Van Nes. 2016. Designing urban spatial vitality from morphological perspective-A study based on quantified urban morphology and activities' testing. Urban Planning International 31 (1).

\section{Further Readings}

Hillier, B., and J. Hanson. 1984. The Social Logic of Space. Cambridge, UK: Cambridge University Press.

Hillier, B. 1996. Space is the machine: a configurational theory of architecture. Cambridge, UK: Cambridge University Press.

Marshall S. 2005. Streets and patterns. Spon Press, Oxon.

Yamu, C., A. van Nes, and C. Garau. 2021. Bill Hillier's legacy: space syntax-a synopsis of basic concepts, measures, and empirical application. Sustainability, 13(6):3394. https://doi.org/10.3390/su13063394

Open Access This chapter is distributed under the terms of the Creative Commons Attribution 4.0 International License (http://creativecommons. org/licenses/by/4.0/), which permits use, duplication, adaptation, distribution and reproduction in any medium or format, as long as you give appropriate credit to the original author(s) and the source, a link is provided to the Creative Commons license and any changes made are indicated.

The images or other third party material in this chapter are included in the work's Creative Commons license, unless indicated otherwise in the credit line; if such material is not included in the work's Creative Commons license and the respective action is not permitted by statutory regulation, users will need to obtain permission from the license holder to duplicate, adapt or reproduce the material. 